COMMUNICATIONS IN

ANALYSIS AND GEOMETRY

Volume 12, Number 2, 417-485, 2004

\title{
Non-integral Toroidal Dehn Surgeries
}

\author{
C. McA. Gordon ${ }^{1}$ And John Luecke ${ }^{2}$
}

\section{Introduction.}

If we perform a non-trivial Dehn surgery on a hyperbolic knot in the 3sphere, the result is usually a hyperbolic 3-manifold. However, there are exceptions: there are hyperbolic knots with surgeries that give lens spaces [1], small Seifert fiber spaces [2], [5], [7], [19], and toroidal manifolds, that is, manifolds containing (embedded) incompressible tori [6], [7]. In particular, Eudave-Muñoz [6] has explicitly described an infinite family of hyperbolic knots $k(\ell, m, n, p)$, each of which has a specific half-integral toroidal surgery. (These are the only known examples of non-trivial, non-integral, non-hyperbolic surgeries on hyperbolic knots.) Here we show that these knots are the only hyperbolic knots with non-integral toroidal surgeries.

Theorem 1.1. Let $K$ be a hyperbolic knot in $S^{3}$ that admits a non-integral surgery containing an incompressible torus. Then $K$ is one of the EudaveMuñoz knots $k(\ell, m, n, p)$, and the surgery is the corresponding half-integral surgery.

The knots $k(\ell, m, n, p)$ have tunnel number $1[6]$, and hence they lie on a genus 2 Heegaard surface in $S^{3}$ and are strongly invertible. The same is true of the Berge knots [1], which are the only known hyperbolic knots with lens space surgeries, and the knots of Dean [5], which have small Seifert fiber space surgeries. (By a small Seifert fibert space we mean one with base surface $S^{2}$ and exactly three exceptional fibers.) Examples of hyperbolic knots with small Seifert fiber space surgeries, that are not Dean knots, have been given by Mattman, Miyazaki and Motegi [19]. They also lie on a genus 2 Heegaard surface, but are not strongly invertible (instead, they are periodic of period 2), and therefore do not have tunnel number 1. In light of the fact that all these knots have a relatively simple structure, Theorem 1.1 suggests that it might eventually be possible to prove analogs for the other two cases

\footnotetext{
${ }^{1}$ Partially supported by NSF Grant DMS-9971718 and TARP Grant 003658-0519-2001.

${ }^{2}$ Partially supported by NSF Grant DMS-9803122.
} 
also, i.e., to prove the Berge Conjecture [1], that any hyperbolic knot with a lens space surgery is a Berge knot, and that any hyperbolic knot with a small Seifert fiber space surgery is obtained by the construction of either Dean, or Mattman, Miyazaki and Motegi.

We remark that modulo the Geometrization Conjecture, if a nonhyperbolic Dehn surgery on a hyperbolic knot is neither a lens space nor a small Seifert fiber space, then it is either $S^{3}, S^{2} \times S^{1}$, a non-prime manifold, or a toroidal manifold. The first happens only when the surgery is trivial [12], the second never happens [8], and the third is conjectured to never happen [9]. Though non-integral toroidal surgeries are classified by Theorem 1.1, the case of integral toroidal surgeries is the least clear; there are many examples, and no good conjecture about their structure.

To describe the proof of Theorem 1.1, which builds on our earlier papers [15] and [16], we briefly recall Eudave-Muñoz' construction. The knots $k(\ell, m, n, p)$ come from certain 2-string tangles $B(\ell, m, n, p)$ in the 3-ball; these have the property that, if $R(r / s)$ denotes the rational tangle with slope $r / s$, then capping $B(\ell, m, n, p)$ off with $R(1 / 0)$ gives the unknot, while capping it off with $R(1 / 2)$ gives a knot that is the union of two prime tangles $\left(B_{1}, t_{1}\right)$ and $\left(B_{2}, t_{2}\right)$. It follows that the 2 -fold branched covering of $B(\ell, m, n, p)$ is the exterior of a strongly invertible knot $k(\ell, m, n, p)$ in the 3 -sphere, which has a half-integral surgery $M$ containing an incompressible torus $\widehat{T}$. The torus $\widehat{T}$ separates $M$ into $M_{1}$ and $M_{2}$, where $M_{i}$ is the 2-fold branched covering of $\left(B_{i}, t_{i}\right), i=1,2$. Each of the tangles $\left(B_{i}, t_{i}\right)$ turns out to be a Montesinos tangle of length 2 (i.e., a sum of two rational tangles), and therefore each $M_{i}$ is a Seifert fiber space over the disk with two exceptional fibers. Furthermore, the Seifert fibers of $M_{1}$ and $M_{2}$ intersect exactly once on $\widehat{T}$, and one of the four exceptional fibers has multiplicity 2 . It also turns out that the 2 -sphere $S$ separating the knot $B(\ell, m, n, p) \cup R(1 / 2)$ into $\left(B_{1}, t_{1}\right)$ and $\left(B_{2}, t_{2}\right)$ intersects the tangle $R(1 / 2)$ in a single disk. This implies that the torus $\widehat{T}$, which is the 2 -fold branched covering of $S$, meets the 2 -fold branched covering of $R(1 / 2)$ in two meridian disks, i.e., $\widehat{T}$ meets the core of the surgery solid torus in two points.

In [15] we showed that if a non-integral surgery on a hyperbolic knot $K$ in $S^{3}$ gives a toroidal 3-manifold $M$, then firstly, the surgery is half-integral, and secondly, an incompressible torus $\widehat{T}$ in $M$ that meets the core of the surgery solid torus minimally does so in either two or four points. The second possibility was eliminated in [16]. It was also shown in [15] that if $M_{1}$ and $M_{2}$ are the two components into which $\widehat{T}$ separates $M$, then $M_{1}$, say, is a Seifert fiber space over the disk with two exceptional fibers. 
This is already enough to show that $K$ arises from the same general construction used by Eudave-Muñoz: the exterior of $K$ is the 2-fold branched covering of a 2-string tangle $(B, t)$, with the property that $(B, t) \cup R(1 / 0)$ is the unknot, and, for some integer $r,(B, t) \cup R(r / 2)$ is the union of two prime tangles $\left(B_{1}, t_{1}\right)$ and $\left(B_{2}, t_{2}\right)$, where the decomposing sphere $S$ meets $R(r / 2)$ in a single disk. Moreover, $\left(B_{1}, t_{1}\right)$, say, is a sum of two rational tangles. In the present paper we carry out a more refined analysis of the situation, and show that the tangle $(B, t)$ must in fact be one of Eudave-Muñoz' tangles $B(\ell, m, n, p)$.

Our starting point, as in [15] and [16], is to consider the labelled intersection graphs $G_{Q}$ and $G_{T}$ on a suitably chosen Heegaard 2-sphere $\widehat{Q}$ in $S^{3}$ and a suitably chosen incompressible torus $\widehat{T}$ in $M$, respectively. Because the graph $G_{T}$ does not represent all types [12], [15], the graph $G_{Q}$ contains a certain kind of subgraph, $\Lambda$, which we call a great web; see [15]. In Section 2 we show (Theorem 2.1) that $G_{Q}$ contains a great web $\Lambda$ with an additional technical property, namely that its ghost edges are extremal in the corresponding subgraph of $G_{T}$; see Section 2 for definitions.

Since all the vertices of $\Lambda$ have the same sign, the edges of $G_{T}$ corresponding to the edges in the boundary of a face $f$ of $\Lambda$ will have an endpoint at each of the two vertices of $G_{T}$. They therefore belong to at most four parallelism classes on $G_{T}$. We say that the face $f$ is good if its edges belong to exactly two such edge classes, and moreover, one of these classes has the property that no two adjacent edges in the boundary of $f$ belong to that class. In Section 4 we show that the existence of a good face of $\Lambda$, lying in $M_{i}(i=1$ or 2$)$, implies that $M_{i}$ is a Seifert fiber space over the disk with two exceptional fibers; see Theorem 4.1. In Section 3 we show (Theorem 3.1) that $\Lambda$ contains a good face $f_{i}$ that lies in $M_{i}$ for $i=1$ and 2 . Hence $M_{1}$ and $M_{2}$ are both Seifert fiber spaces over the disk with two exceptional fibers. The proof of Theorem 3.1 involves a detailed analysis of the dual graph of $\Lambda, \Lambda^{*}$, with various dual orientations, in which an edge of $\Lambda^{*}$ is oriented according to the edge class of the corresponding dual edge of $\Lambda$. In this way we arrange, for example, that a sink or source vertex of $\Lambda^{*}$ is dual to a face of $\Lambda$ whose edges belong to at most two edge classes.

The existence of the good faces $f_{1}$ and $f_{2}$ leads to a description of the exterior of $K$, in terms of Dehn surgery on a certain link in $S^{3}$; this is done in Section 5. First, we see that the Seifert fibers of $M_{1}$ and $M_{2}$ intersect exactly once on $\widehat{T}$. It follows that we can regard $M$-(neighborhoods of the four exceptional fibers) as the exterior of the 4-component link $L_{0}$ in $S^{3}$ which is obtained from the Hopf link by adding a nearby 0 -framed parallel 
copy of each component. We may therefore identify $M$ with a certain Dehn surgery on the link $L_{0}$. A further analysis, using the faces $f_{1}$ and $f_{2}$, allows us to explicitly determine a fifth component $K_{0}$ in $S^{3}$ that corresponds under this identification to the core of the surgery solid torus in $M$. Thus the exterior of $K$ is obtained from the exterior of $K_{0}$ in $S^{3}$ by Dehn surgery on $L_{0}$. Letting $L=L_{0} \cup K_{0}$, this is the statement of Proposition 5.6. We remark that $L$ has the same exterior as the link called the minimally twisted 5-chain; thus $K$ can also be thought of as obtained by surgery on this link. The link $L$ is strongly invertible, by an involution $h$. Taking a $h$-invariant tubular neighborhood $N(L)$ of $L$, the quotient under $h$ of $N(L) \cup \operatorname{Fix}(h)$ is an embedding in $S^{3}$ of the complete graph on five vertices, with the vertices thickened to 3-balls. Removing the interiors of these balls we get a pair $\mathcal{P}$ consisting of arcs in a 5 -punctured $S^{3}$, with four arc-endpoints on each boundary 2 -sphere. We call $\mathcal{P}$ the pentangle (the terminology is due to John Conway).

If $\alpha, \beta, \gamma, \delta, \varepsilon \in \mathbb{Q} \cup\{1 / 0\}$, then inserting the rational tangles $R(\alpha)$ etc. into the punctures of $\mathcal{P}$ gives a knot or link $\mathcal{P}(\alpha, \beta, \gamma, \delta, \varepsilon)$, whose 2 -fold branched covering is obtained by doing Dehn surgery on the components of $L$, the surgery solid tori being the 2-fold branched coverings of $R(\alpha)$, etc.. If we parametrize slopes on $\partial N\left(K_{0}\right)$ so that the slope of the toroidal filling $M$ is $0 / 1$, and the preferred longitude of $K_{0}$ in $S^{3}$ is $1 / 0$, then a careful examination of the faces $f_{1}$ and $f_{2}$ shows that the meridian $\mu$ of $K$ is $2 / 1$. Thus if $\alpha, \beta, \gamma$ and $\delta$ are the slopes of the Dehn surgeries on the components of $L_{0}$ that give $M$, then $\mathcal{P}\left(\alpha, \beta, \gamma, \delta, \frac{2}{1}\right)$ is the unknot, and $\mathcal{P}\left(\alpha, \beta, \gamma, \delta, \frac{0}{1}\right)$ is the union of two Montesinos tangles of length 2 . Let $\mathcal{Q}$ be obtained by filling in the last boundary component of $\mathcal{P}$ with the rational tangle $R(2 / 1)$; then $\mathcal{Q}(\alpha, \beta, \gamma, \delta)$ is the unknot. Since the surgeries on the components of $L_{0}$ give rise to the exceptional fibers of $M_{1}$ and $M_{2}$, we also have that $\Delta\left(\chi, \frac{1}{0}\right) \geq 2$ for $\chi \in\{\alpha, \beta, \gamma, \delta\}$. We show (Proposition 6.1) that these conditions imply that $\frac{1}{2} \in\{\alpha, \beta, \gamma, \delta\}$. This is proved by noting that if one of the tangle co-ordinates of a tangle filling of $\mathcal{Q}$ takes certain special values, then the corresponding links have particularly simple descriptions. These computations (expressed in terms of the 2-fold branched coverings) are carried out in Section 7. In Section 8 we apply theorems about distances between various kinds of Dehn fillings, using the fillings on the 2 -fold branched covering of $\mathcal{Q}$ described in Section 7, to get restrictions on $\alpha, \beta, \gamma, \delta$ which enable us to prove Proposition 6.1

The proof of Theorem 1.1 (given at the end of Section 6) is now completed as follows. We may assume, by symmetry, that $\delta=1 / 2$. Filling in the corresponding puncture of $\mathcal{P}$ with $R(1 / 2)$ gives a tangle $\mathcal{B}$, such that 
$\mathcal{B}\left(\alpha, \beta, \gamma, \frac{2}{1}\right)$ is the unknot. Now the tangles $B(\ell, m, n, p)$ are all of the form $\mathcal{B}(A, B, C$,*), for some $A, B, C \in \mathbb{Q}$. Moreover, in anticipation of this kind of approach to Theorem 1.1, Eudave-Muñoz shows in [7] that these triples $(A, B, C)$ are the only ones with the property that $\mathcal{B}\left(A, B, C, \frac{2}{1}\right)$ is the unknot. We conclude that our tangle $(B, t)=\mathcal{P}\left(\alpha, \beta, \gamma, \frac{1}{2}, *\right)$ is one of the tangles $B(\ell, m, n, p)$.

In the Appendix we prove the analog of Theorem 1.1 for knots in solid tori: if $K$ is a hyperbolic knot in a solid torus $N$ having a non-integral Dehn surgery that contains an essential torus, then there is an Eudave-Muñoz knot $k(\ell, m, n, p)$ and an unknotted simple closed curve $c$ in $S^{3}-k(\ell, m, n, p)$ (see [6], [20]) such that $(N, K) \cong\left(S^{3}-\operatorname{Int} N(c), k(\ell, m, n, p)\right)$.

\section{2. $G_{T}, G_{Q}$ and the existence of a web in $G_{Q}$.}

Let $K$ be a hyperbolic knot in the 3 -sphere, with exterior $E(K)=S^{3}-$ Int $N(K)$. Let $K(\tau)$ be the result of $\tau$-Dehn surgery on $K$; thus $K(\tau)=$ $E(K) \cup V$, where $V$ is a solid torus that is glued to $E(K)$ along the boundary in such a way that the slope $\tau$ on $\partial E(K)$ bounds a meridian disk in $V$. Let $K_{\tau}$ denote the core of $V$. Assume that $K(\tau)$ contains an incompressible torus, and that $\tau$ is a non-integral slope, i.e. $\Delta(\tau, \mu) \geq 2$, where $\mu$ is the meridian of $K$. By [15], $\Delta(\tau, \mu)=2$; thus, expressing $\tau$ in terms of meridian-longitude co-ordinates on $\partial E(K)$ in the usual way, $\tau$ is of the form $p / 2$. Let $\widehat{T}$ be an essential torus in $K(p / 2)$ that intersects $K_{\tau}$ minimally. Then Theorem of [16] says that $T=\widehat{T} \cap E(K)$ is a properly embedded, incompressible, twicepunctured torus in $E(K)$, where each component of $\partial T$ represents slope $p / 2$ on $\partial E(K)$.

Let $p_{1}, p_{2}$ be two points in $S^{3}$. We can write $S^{3}-\left\{p_{+}, p_{-}\right\}=\widehat{Q} \times(-1,1)$, where $\widehat{Q}$ is a 2 -sphere. Lemma 4.4 of $[8$, p.491] says that we can find a $\widehat{Q}=\widehat{Q} \times\{i\}$ for some $i$ such that

(1) $\widehat{Q}$ intersects $K$ transversely. Thus $Q=\widehat{Q} \cap E(K)$ is a properly embedded planar surface in $E(K)$ such that each component of $\partial Q$ is a copy of the meridian of $K$.

(2) $Q$ intersects $T$ transversely and no arc component of $Q \cap T$ is parallel in $Q$ to $\partial Q$ or parallel in $T$ to $\partial T$.

Let $G_{Q}$ be the graph in $\widehat{Q}$ obtained by taking as the (fat) vertices the disks $\widehat{Q}$ - Int $Q$ and as edges the arc components of $Q \cap T$ in $\widehat{Q}$. Similarly, $G_{T}$ is the graph in $\widehat{T}$ whose vertices are the disks $\widehat{T}-\operatorname{Int} T$ and whose 

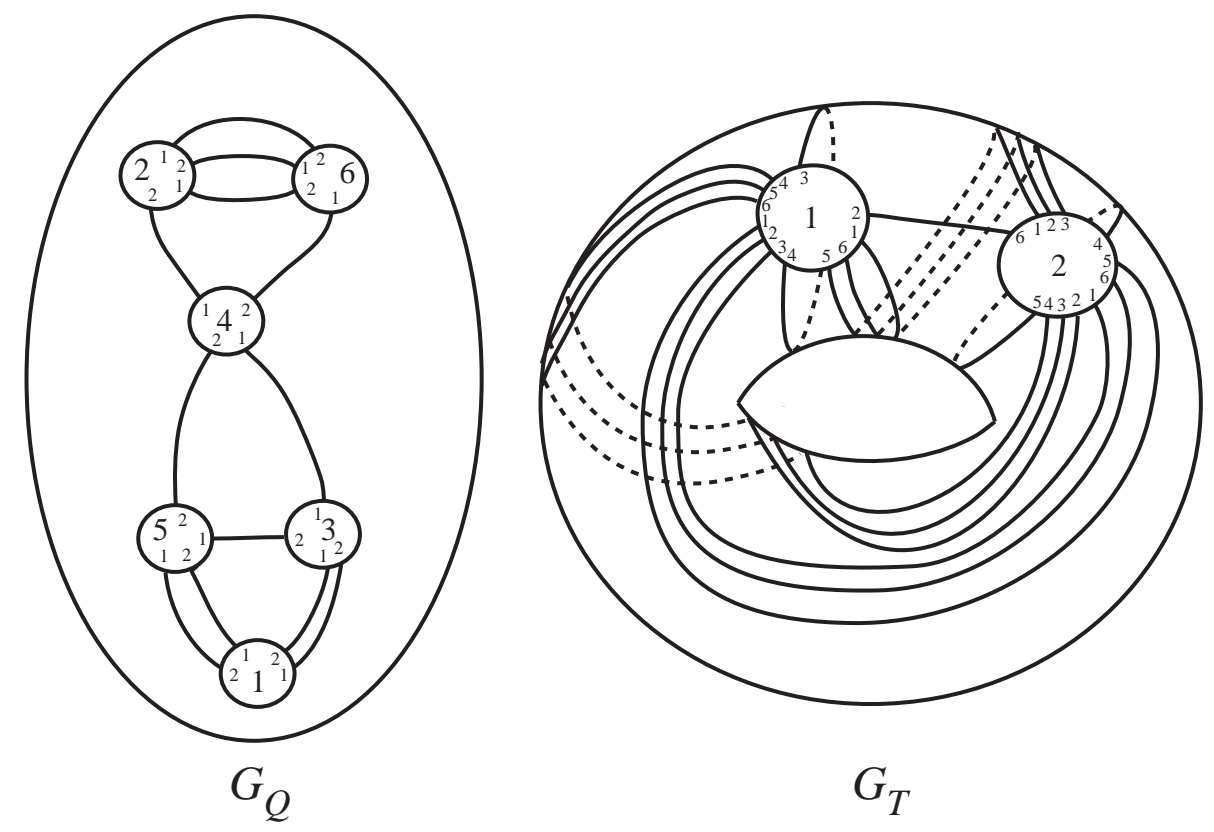

Figure 2.1.

edges are the arc components of $Q \cap T$ in $\widehat{T}$. We number the components of $\partial Q 1,2, \ldots, q$ in the order they appear in $\partial E(K)$. Similarly we number the components of $\partial T, 1,2$. This gives a numbering of the vertices of $G_{Q}$ and $G_{T}$. For example, an endpoint of an edge in $G_{Q}$ at vertex $x$ will be labelled $y$ if the endpoint represents the intersection of component $x$ of $\partial Q$ with component $y$ of $\partial T$. On a vertex of $G_{Q}\left(G_{T}\right)$ one sees the labels 1 and 2 (1 through $q$ respectively) appearing in order around the vertex, each label appearing exactly 2 times. See Figure 2.1. Two vertices on $G_{Q}$ are parallel if the ordering of the labels on each is clockwise or the ordering on each is anticlockwise, otherwise the vertices are anti-parallel. The same applies to vertices of $G_{T}$. In particular, because $\widehat{T}$ is separating, vertices 1 and 2 of $G_{T}$ are anti-parallel. The graphs of $G_{Q}$ and $G_{T}$ then satisfy the following parity rule: an edge connects parallel vertices on one graph if and only if it corrects anti-parallel vertices on the other (this follows from orientability).

A loop edge of $G_{T}$ is one with both endpoints on the same vertex. There must be the same number of loop edges at vertex 1 on $G_{T}$ as there are loop edges at vertex 2 . This implies that two loop edges based at the same vertex in $G_{T}$ are isotopic relative to the fat vertices of $G_{T}$. There are at most four isotopy classes of edges connecting vertices 1 and 2 on $G_{T}$. Thus 


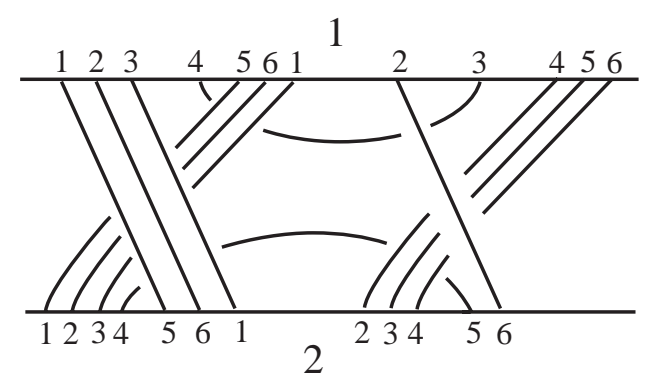

Figure 2.2.

the graph $G_{T}$ will, up to homeomorphism, be of the form illustrated in Figure 2.1, with $q$ varying and possibly some of the isotopy classes being empty. For convenience we will represent the graph $G_{T}$ more schematically, as in Figure 2.2, which shows the graph $G_{T}$ of Figure 2.1.

Let $\Gamma$ be a subgraph of $G_{Q}$. A ghost edge of $\Gamma$ is an edge of $G_{Q}$ that is incident to at least one vertex of $\Gamma$ but is not an edge of $\Gamma$. A ghost endpoint of $\Gamma$ is an endpoint at a vertex of $\Gamma$ of a ghost edge of $\Gamma$. Following [15] we define a web, $\Lambda$, of $G_{Q}$ to be a connected subgraph of $G_{Q}$ whose vertices are all parallel and such that $\Lambda$ has at most 2 ghost endpoints. We will refer to a disk $D=\widehat{Q}-U$, where $\mathrm{U}$ is a component of $\widehat{Q}-\operatorname{nhd}(\Lambda)$, as a disk bounded by $\Lambda$. A great web in $G_{Q}$ is a web with the property that there is a disk bounded by $\Lambda, D_{\Lambda}$, such that $\Lambda$ contains all the edges of $G_{Q}$ that lie in $D_{\Lambda}$.

There is a one-one correspondence between the edges of $G_{Q}$ and $G_{T}$. For any subgraph $\Gamma$ of $G_{Q}$, let $\Gamma_{T}$ be the corresponding subgraph of $G_{T}$. We shall say that an edge of $G_{Q}$ is extremal with respect to $\Gamma$ if the corresponding edge of $G_{T}$ does not lie strictly between parallel edges of $\Gamma_{T}$ (edges are parallel if they lie in the same isotopy class relative to $\partial T$ ).

Theorem 2.1. $G_{Q}$ contains a great web $\Lambda$ such that all ghost edges of $\Lambda$ are extremal with respect to $\Lambda$.

For the proof of this theorem we will assume familiarity with the notation of $[12]$.

Proof. Let $G_{Q}^{\prime}$ be an innermost component of $G_{Q}$, and let $D$ be a disk in $\widehat{Q}$ such that $G_{Q} \cap D=G_{Q}^{\prime}$. From now on we will restrict our attention to $G_{Q}^{\prime}$ and the corresponding subgraph $G_{T}^{\prime}$ of $G_{T}$, and hence for notational convenience we will rename $G_{Q}^{\prime}, G_{T}^{\prime}$ as $G_{Q}, G_{T}$ respectively. By Theorem 2.1 of [15], $G_{T}$ does not represent some type $\tau$. 
Case I. $\tau$ is trivial. Then there is at most one loop edge of $G_{T}$ incident to vertex 1 , and at most one loop edge incident to vertex 2 . By the parity rule, all other edges of $G_{T}$ correspond to edges of $G_{Q}$ connecting parallel vertices. Consider the subgraph of $G_{Q}$ consisting of all such edges, and let $\Lambda$ be an innermost component of this subgraph. Then $\Lambda$ has at most two ghost edges (corresponding to loop edges of $G_{T}$ ), each meeting $\Lambda$ in a single endpoint. Hence $\Lambda$ is a great web, and the ghost edges of $\Lambda$ are extremal with respect to $\Lambda$, since the edges of $\Lambda$ correspond to edges of $G_{T}$ connecting vertices 1 and 2, while the ghost edges of $\Lambda$ correspond to loop edges of $G_{T}$.

This proves the theorem in Case I.

Case II. $\tau$ is non-trivial. Follow the proof on p.406 of [12], case (2), to construct a sequence of stars $T_{1}, T_{2}, \ldots, T_{n}, n \geq 1$, such that

(i) $\left[T_{1}\right]=\tau,\left[T_{i}\right]$ is non-trivial, $1 \leq i \leq n$;

(ii) $T_{i}=d_{i} T_{i-1}$, where $d_{i}=d^{ \pm}, 2 \leq i \leq n$;

(iii) all elements of $C\left(T_{n}\right)$ have the same parity;

(iv) all elements of $A\left(T_{n}\right)$ have the same parity.

Set $L_{i}=L\left(T_{i}\right)$. Assume that $f$ is a face of $G_{T}\left(L_{n}\right)$ representing $\left[T_{n}\right]$. Then $(n-1)$ applications of Corollary 2.4.2 of [12] show that $f$ contains a face $f^{\prime}$ of $G_{T}\left(L_{1}\right)=G_{T}$ representing $\tau$. Thus if we have a face of $G_{T}\left(L_{n}\right)$ representing $\left[T_{n}\right]$ we are done. So we assume there are no such faces.

Let $s_{c}$ (resp. $s_{a}$ ) be the number of edges of $\Gamma\left(T_{n}\right)$ whose endpoints are both in $C\left(T_{n}\right)$ (resp. $A\left(T_{n}\right)$ ); we refer to these as clockwise (resp. anticlockwise) switch edges. Let $s=s_{c}+s_{a}$ be the total number of switch edges. Let $S$ be the number of switches around $T_{n}$. That is $S=2\left(\left|C\left(T_{n}\right)\right|+\left|A\left(T_{n}\right)\right|\right)=$ $4\left|C\left(T_{n}\right)\right|$. (See pp.602 and 603 of [15].) Note that $C\left(T_{n}\right)$ and $A\left(T_{n}\right)$ are non-empty since $\left[T_{n}\right]$ is non-trivial.

Let $i=S / 2-1$. The proof of Lemma 2.3 .2 of [12] shows that $s \geq i t$, where $t$ is the number of vertices of $G_{T}$. Here $t=2$.

Lemma 2.2. $s_{c}$ and $s_{a}$ are both $\leq i t / 2$.

Proof. Let $\Lambda_{c}$ (resp. $\Lambda_{a}$ ) be the subgraph of $G_{Q}$ consisting of all vertices of $G_{Q}$ in $C\left(T_{n}\right)$ (resp. $A\left(T_{n}\right)$ ), and all edges of $G_{Q}$ corresponding to clockwise (resp. anticlockwise) switch edges of $\Gamma\left(T_{n}\right)$.

Note that there are $2\left|C\left(T_{n}\right)\right| t$ points where edges of $G_{Q}$ are incident to vertices of $\Lambda_{c}$. Assume $s_{c}>i t / 2$. Then more than $2(i t / 2)=i t=$ 
$(S / 2-1) t=2\left|C\left(T_{n}\right)\right| t-t$ of these points are endpoints of edges of $\Lambda_{c}$. As $t=2$, it follows that $\Lambda_{c}$ has at most one ghost endpoint. But since $T$ is separating, the faces of $G_{Q}$ can be shaded alternately $B$ (black) and $W$ (white), from which it is easy to see that $\Lambda_{c}$ cannot have a single ghost endpoint. Hence $\Lambda_{c}$ has no ghost endpoints. But this contradicts the fact that $G_{Q}$ is connected.

Similarly we see that $s_{a} \leq i t / 2$.

Lemma 2.2 and the fact that $s \geq i t$ implies that $s_{c}=s_{a}=i t / 2$. Thus, defining $\Lambda_{c}\left(\Lambda_{a}\right)$ as above, of the $2\left|C\left(T_{n}\right)\right| t$ edge endpoints at the vertices of $\Lambda_{c}$, exactly $2(i t / 2)=2\left|C\left(T_{n}\right)\right| t-t$ are endpoints of edges of $\Lambda_{c}$. That is, $\Lambda_{c}$ has exactly $t=2$ ghost endpoints. Thus $\Lambda_{c}$ is a web, and furthermore, the ghost edges of $\Lambda_{c}$ do not correspond to switch edges of $\Gamma\left(T_{n}\right)$.

Similarly, $\Lambda_{a}$ is a web whose ghost edges do not correspond to switch edges of $\Gamma\left(T_{n}\right)$.

Note that, as above, no component of $\Lambda_{c}$ or $\Lambda_{a}$ can have a single ghost endpoint, and hence, since $G_{Q}$ is connected, $\Lambda_{c}$ and $\Lambda_{a}$ are connected.

Without loss of generality, assume that $\Lambda_{c}$ is innermost on $D$ among $\Lambda_{c}$ and $\Lambda_{a}$. A complementary component of $\Lambda_{c}$ is a component of $D-\operatorname{nhd}\left(\Lambda_{c}\right)$.

Lemma 2.3. All the vertices of $G_{Q}-\Lambda_{c}$ lie in the same complementary component of $\Lambda_{c}$.

Proof. Let $\Omega$ be the subgraph of $G_{Q}$ consisting of all vertices of $G_{Q}-\Lambda_{c}$ and all edges of $G_{Q}$ connecting these vertices. Since $G_{Q}$ is connected, each component of $\Omega$ must be joined by an edge to $\Lambda_{c}$, hence joined to $\Lambda_{c}$ by one of the two ghost edges of $\Lambda_{c}$. Because $G_{Q}$ can be shaded alternately $B / W$, each component of $\Omega$ must be joined to $\Lambda_{c}$ by at least two ghost edges. Hence $\Omega$ has only one component.

Since $\Lambda_{a}$ lies in the complementary component $U$ of $\Lambda_{c}$ containing $\partial D$, we see that $D^{\prime}=D-U$ is a disk bounded by $\Lambda_{c}$ with $\Omega \cap D^{\prime}=\emptyset$. That is, $\Lambda_{c}$ is a great web.

Lemma 2.4. The ghost edges of $\Lambda_{c}$ are extremal with respect to $\Lambda_{c}$.

Proof. Assume not. Then there are edges $e, e_{1}, e_{2}$ of $G_{T}$ such that $e$ corresponds to a ghost edge of $\Lambda_{c}, e_{1}, e_{2}$ correspond to edges of $\Lambda_{c}$, and $e, e_{1}, e_{2}$ are parallel on $G_{T}$, with $e$ lying between $e_{1}$ and $e_{2}$. See Figure 2.3. 


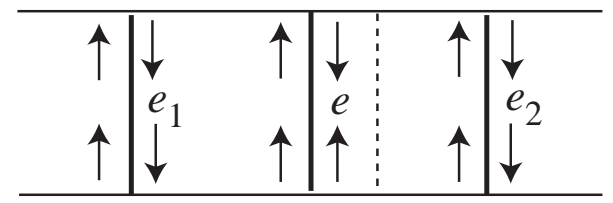

Figure 2.3.

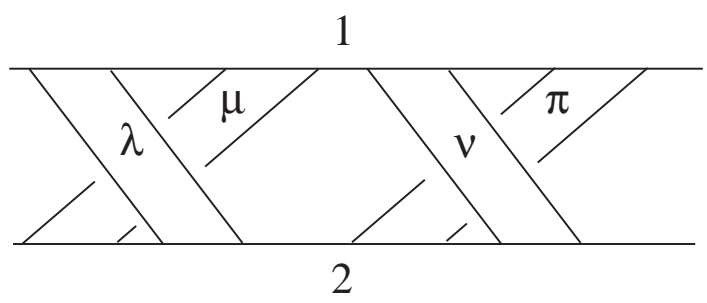

Figure 3.1.

Now $e_{1}, e_{2}$ are clockwise switch edges of $\Gamma\left(T_{n}\right)$, and $e$ has a clockwise switch at exactly one endpoint (since $e$ corresponds to an edge of $G_{Q}$ that has exactly one endpoint in $\Lambda_{c}$ ). But this implies that one of the (bigon) faces of $G_{T}\left(L_{n}\right)$ contiguous to $e$ is a sink or source of $\Gamma\left(T_{n}\right)$. See Figure 2.3. This contradicts our assumption that $G_{T}\left(L_{n}\right)$ does not represent $\left[T_{n}\right]$.

Lemma 2.4 shows that $\Lambda_{c}$ is a web with the desired properties, completing the proof of Theorem 2.1.

\section{Analyzing $G_{Q}$.}

We will call one side of $\widehat{T}$ in $K(p / 2)$ the $W$ (hite) side and the other the $B$ (lack) side. Then $T$ has a $W$ and $B$ side in $E(K)$. The faces of $G_{Q}$ are called $W$ or $B$ according to which side of $T$ a neighborhood of their boundary lies. In this section we will show the existence of a $B$ face and of a $W$ face of a special form (a good binary face) which we will use in the next section to prove that the $B$ and $W$ sides of $\widehat{T}$ are Seifert fiber spaces over the disk with two exceptional fibers.

The edge class of an edge of $G_{T}$ is its isotopy class in $\widehat{T}$ relative to the fat vertices of $G_{T}$. There are most four different edge classes of edges connecting vertex 1 to vertex 2 (the non-loop edge classes). We label these edge classes $\lambda, \mu, \nu, \pi$ as in Figure 3.1. Note that loop edge classes are disregarded in this labelling.

Two of those (non-loop) edge classes are said to be adjacent if those 
labels at vertex 1 (hence vertex 2 as well) are not separated on vertex 1 by the labels of a third non-loop edge class. They are non-adjacent otherwise. Thus if all four edge classes are represented in $G_{T}$, the pair $\{\lambda, \nu\}$ and the pair $\{\mu, \pi\}$ are non-adjacent.

Let $\varepsilon, \delta$ be two elements of $\{\lambda, \mu, \nu, \pi\}$. Then an $(\varepsilon, \delta)$ face of $G_{Q}$ is one whose edges lie in classes $\varepsilon, \delta$ on $G_{T}$. Lemma 3.1 of [15] shows that any such face must have edges in both classes $\varepsilon$ and $\delta$. We call any such face a binary face of $G_{Q}$.

An $(\varepsilon, \delta)$ face $f$ is $\varepsilon$-good if no two consecutive edges in the boundary of $f$ belong to class $\varepsilon$. An $(\varepsilon, \delta)$ face is good if it is either $\varepsilon$-good or $\delta$-good. A bad face is a binary face that is not good. To indicate the coloring of an $(\varepsilon, \delta)$ face we will often call it an $X(\varepsilon, \delta)$ face where $X$ is to take the value $B$ or $W$.

The following is the main theorem of this section. Its proof appears at the end of the section, after the requisite lemmas have been established.

Theorem 3.1. Let $\Lambda$ be the web described in Theorem 2.1. Then there are distinct edge classes $\varepsilon, \delta_{1}, \delta_{2}$ such that $\Lambda$ contains faces $f_{1}$ and $f_{2}$, of opposite colors, such that $f_{i}$ is a good $\left(\varepsilon, \delta_{i}\right)$ face, $i=1,2$. Moreover at least one of these two faces has length 2 or 3.

If $c$ is a $B$ corner of $G_{Q}$ for which the edge incident to label 1 is in class $\varepsilon$ and the edge incident to label 2 is in class $\delta$, we call it an $B(\varepsilon, \delta)$ corner. Had $c$ been a $W$ corner, we would call it a $W(\varepsilon, \delta)$ corner. We will often describe a corner as $X(\varepsilon, \delta)$ where it is understood that $X$ is to take the value $B$ or $W$.

A bad $X(\varepsilon, \delta)$ face of $G_{Q}, f$, must contain $X(\varepsilon, \varepsilon), X(\delta, \delta), X(\varepsilon, \delta)$, $X(\delta, \varepsilon)$ corners. Define $v_{\varepsilon}(f)$ to be some vertex in the boundary of $f$ at which $f$ has an $X(\varepsilon, \varepsilon)$ corner.

We will repeatedly apply the following principle in the analysis below.

Lemma 3.2. Let $c_{1}, c_{2}, \ldots, c_{n}$ be a collection of $X$ corners of $G_{Q}, X \in$ $\{B, W\}$. The anticlockwise ordering of the endpoints of the edges of these corners on vertex 1 of $G_{T}$ is the same as the clockwise ordering of the edge endpoints on vertex 2 of $G_{T}$. See Figure 3.2.

Proof. This follows from considering the arcs defined by the corners that run from boundary component 1 to boundary component 2 of $T$ along $\partial E(K)$. 

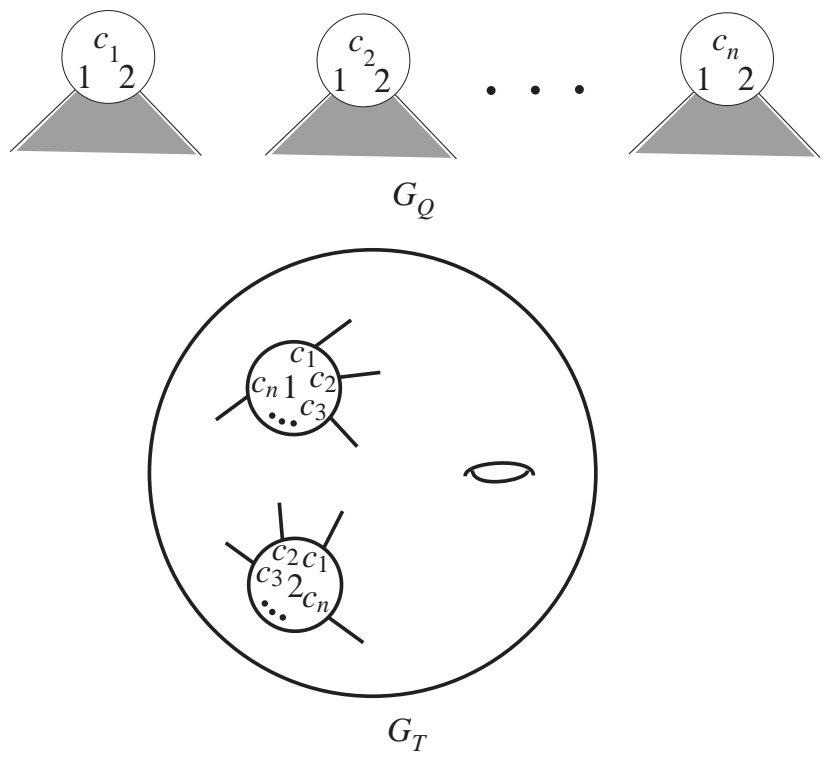

Figure 3.2.

Lemma 3.3. $G_{Q}$ cannot contain a vertex $v$ with two edges in the same edge class incident to $v$ at the same label. See Figure 3.3.

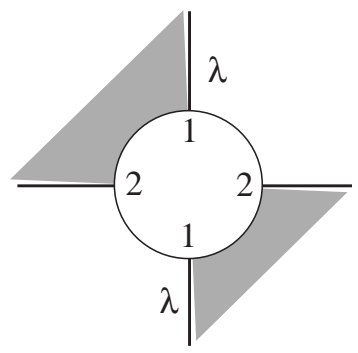

Figure 3.3.

Proof. This would imply that $G_{T}$ had $q+1$ parallel edges in the given edge class. Then one obtains the contradiction that $K$ is a cable knot, using the construction of [11].

Lemma 3.4. Given $X \in\{B, W\}, G_{Q}$ does not contain $X(\varepsilon, \varepsilon)$ corners for three distinct edge classes $\varepsilon$. See Figure 3.4. 


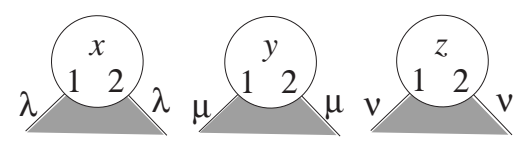

Figure 3.4.

Proof. Suppose, without loss of generality, that $G_{Q}$ contains $B(\lambda, \lambda), B(\mu, \mu)$ and $B(\nu, \nu)$ corners, at vertices $x, y$ and $z$ respectively.

Then the ordering of labels $x, y, z$ at vertices 1 and 2 of $G_{T}$ contradict Lemma 3.2; see Figure 3.5.

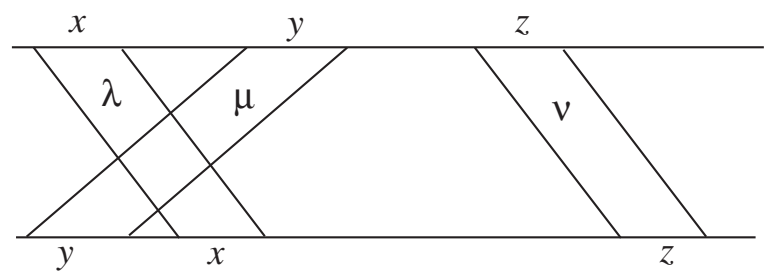

Figure 3.5.

Lemma 3.5. Suppose that $G_{Q}$ contains a bad $X(\lambda, \mu)$ face. Then there exists $\varepsilon \in\{\lambda, \mu\}$ such that, for any edge $e$ of $G_{Q}$ in class $\nu$ or $\pi$, the edges of $G_{Q}$ adjacent to $e$ on the $X$-side are in class $\varepsilon$. See Figure 3.6. More generally, the analogous statement holds whenever $\{\lambda, \mu\}$ is replaced by any pair of adjacent edge classes.

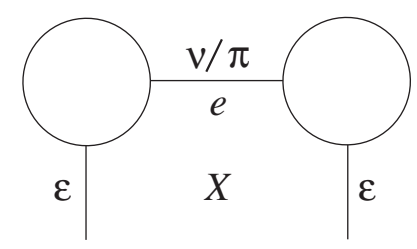

Figure 3.6.

Proof. Suppose, without loss of generality, that $G_{Q}$ contains a bad $B(\lambda, \mu)$ face, $f$. Then $f$ has $B(\lambda, \lambda), B(\mu, \mu), B(\lambda, \mu)$ and $B(\mu, \lambda)$ corners, at vertices $u, v, x, y$, say. See Figure 3.7. 


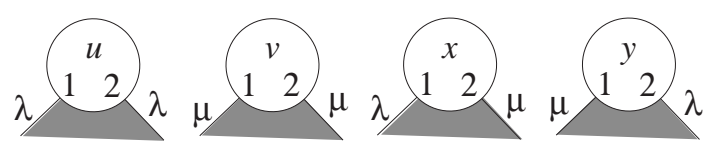

Figure 3.7.

Then, on $G_{T}$, at vertex 1 we have labels $u, x$ in class $\lambda, v, y$ in class $\mu$, and at vertex 2, we have $u, y$ in class $\lambda$, and $v, x$ in class $\mu$. Applying Lemma 3.2, we see that there are two possible arrangements:

(i)

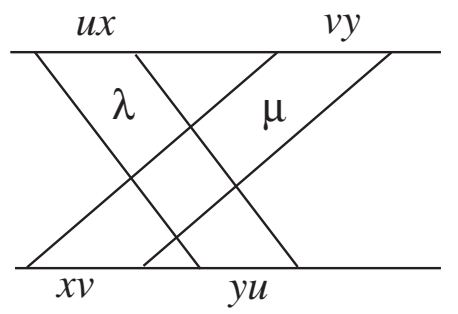

(ii)

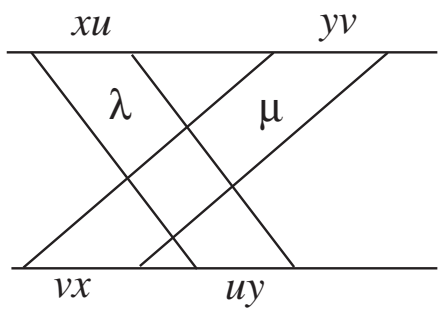

Consider case (i). Let $e$ be an edge of $G_{Q}$ in class $\nu$ or $\pi$, joining vertices $s$ and $t$ as shown:

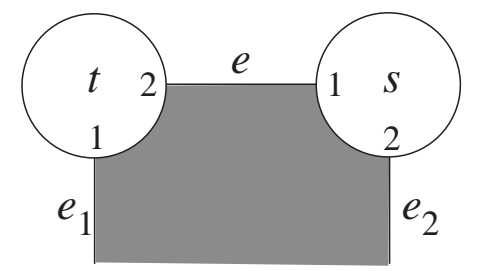

Figure 3.8.

Then, at vertex 1 of $G_{T}$, the label $s$ lies between $y$ and $u$, and hence at vertex 2 is forced to lie in class $\lambda$. Similarly at vertex $2, t$ lies between $u$ and $x$, and hence at vertex 1 lies in class $\lambda$. Thus $e_{1}$ and $e_{2}$ are both in class $\lambda$.

Case (ii) is completely analogous, with $e_{1}$ and $e_{2}$ now being forced to belong to class $\mu$. 
Lemma 3.6. $G_{Q}$ does not contain bad faces of the same color on distinct edge class pairs.

Proof. This would give rise to $X(\varepsilon, \varepsilon)$ corners for three distinct edges classes $\varepsilon$, contradicting Lemma 3.4.

Lemma 3.7. If $G_{Q}$ contains a good $X(\varepsilon, \delta)$ face then any binary $X$-face of $G_{Q}$ on an edge class pair distinct but not disjoint from $\{\varepsilon, \delta\}$ is bad.

Proof. Assume for contradiction that there is a good $X(\varepsilon, \delta)$ face and a good $X\left(\varepsilon, \delta^{\prime}\right)$ face. Applying Theorem 4.1 from section 4 to each of these faces expresses the $X$-side of $\widehat{T}$ as a Seifert fiber space over the disk with two exceptional fibers in two different ways; in particular the Seifert fibers from the two fibrations are not isotopic on $\widehat{T}$. But for such manifolds the Seifert fibration is unique.

We want to define a dual graph to $\Lambda$. To do this, it is convenient to regard $\Lambda$ as a graph in $S^{2}$, rather than $D^{2}$. However, by a face of $\Lambda$ we will still mean a face of $\Lambda$ as a graph in $D^{2}$; so in $S^{2}$, we now have the faces of $\Lambda$ together with an additional outside face. The dual graph $\Lambda^{*}$ of $\Lambda$ is the graph in $S^{2}$ defined in the standard way: choose a point in the interior of each face of $\Lambda$, and a point in the interior of the outside face; these are the vertices of $\Lambda^{*}$; and, for every edge $e$ of $\Lambda$, there is a dual edge $e^{*}$ of $\Lambda^{*}$, joining the vertices of $\Lambda^{*}$ corresponding to the faces of $\Lambda$ (or the outside face) on either side of $e$, and meeting $e$ transversely in a single point.

Let $\left\{E_{+}, E_{-}\right\}$be a partition of the set of edge classes $\{\lambda, \mu, \nu, \pi\}$ into two sets of two elements. Then we can orient the edges of $\Lambda^{*}$ according to the following rule:

an edge of $\Lambda^{*}$ dual to an edge of $\Lambda$ in an edge class in $E_{+}$(resp. $\left.E_{-}\right)$ is oriented from the $W$-side to the $B$-side (resp. from the $B$-side to the $W$-side).

Note. For an edge $e$ of $\Lambda$ in the boundary of the outside face, we regard the side of $e$ contained in the outside face as being locally colored with the color opposite to that of the face of $\Lambda$ that contains $e$.

We shall refer to such an orientation of the edges of $\Lambda^{*}$ as a dual orientation. 
Up to sign (i.e. interchanging $E_{+}$and $E_{-}$), there are exactly three dual orientations, $\omega, \omega^{\prime}$ and $\omega^{\prime \prime}$, shown in Figure 3.9.

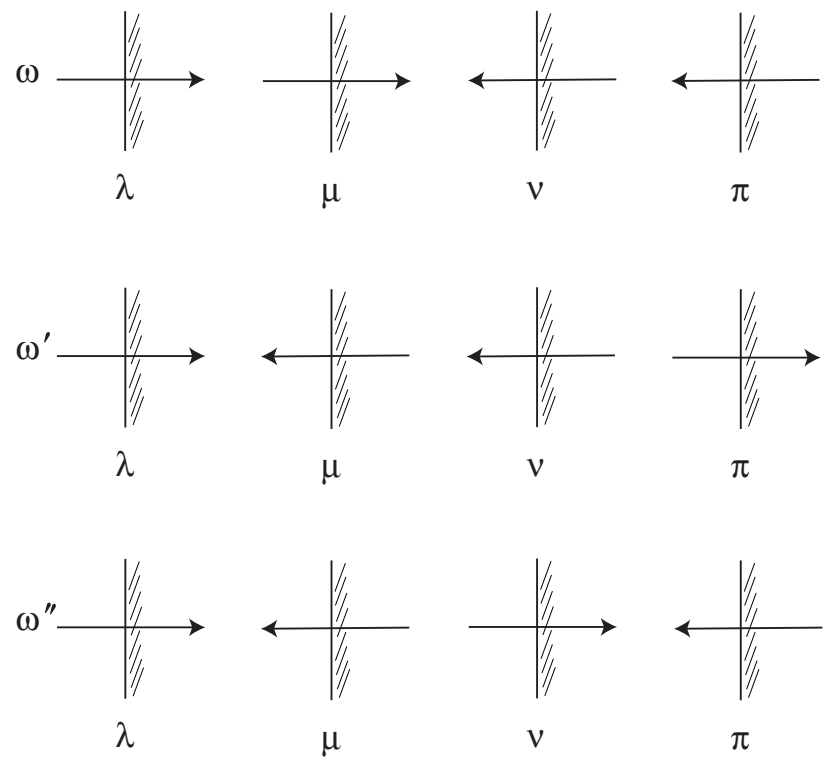

Figure 3.9.

Note that for $\omega$ and $\omega^{\prime}$, the two edges classes in $E_{ \pm}$are adjacent, while for $\omega^{\prime \prime}$ they are non-adjacent.

Consider some dual orientation on $\Lambda^{*}$. Recall the definition of the index of a vertex or face of a graph in $S^{2}$ with oriented edges from Section 2.3 of [12]. That is, the index of a vertex, $v$, is $1-s(v) / 2$, where $s(v)$ is the number of switches at $v$. In particular, a sink or source is a vertex of index 1 , and a cycle is a face of index 1. A cycle of $\Lambda^{*}$, with respect to the given dual orientation, is dual to a vertex $v$ of $\Lambda$. If $v$ is an exceptional vertex of $\Lambda$, i.e., a ghost edge of $\Lambda$ is incident to $v$, we will call the cycle exceptional; otherwise we will call it ordinary. We will use the following notation:

$s=$ the number of sinks and sources of $\Lambda^{*}$ at vertices dual to faces of $\Lambda$ (i.e. we exclude the outside face);

$c=$ the number of ordinary cycles of $\Lambda^{*}$;

$c_{e}=$ the number of exceptional cycles of $\Lambda^{*}$;

$d=$ the index of the outside face; 
$t=$ the number of cycles of $\Lambda^{*}$ of index -1 .

Lemma 3.8. (1) $s+c+c_{e}+d \geq 2+t$;

(2) if $d=1$ then $c_{e}=0$;

(3) if $c_{e}+d \geq 2$ then $d=0, c_{e}=2$, and the two exceptional cycles are oppositely oriented;

(4) if $s+c=0$ then $c_{e}=2$ and the two exceptional cycles are oppositely oriented.

Proof. (1) This is Lemma 2.3.1 of [12].

(2) A cycle dual to an exceptional vertex gives one dual edge oriented into the outside face, and another oriented outwards from it. Hence $d \leq 0$.

(3) Suppose $c_{e}+d \geq 2$. Since $c_{e} \leq 2$, (there are at most two exceptional vertices) we have $d=0$ or 1 . If $d=1$ then $c_{e}=0$ by (2), contradicting $c_{e}+d \geq 2$. Hence $d=0$ and $c_{e}=2$.

If the two exceptional cycles were coherently oriented, e.g. both clockwise, then clearly $d \leq-1$. $(3)$.

(4) By (1), $s+c=0$ implies $c_{e}+d \geq 2$, and the result now follows from

Lemma 3.9. Suppose that $\Lambda^{*}$ contains an ordinary cycle with respect to $\omega$. Then either

(1) every $B$-face of $\Lambda$ is a $(\lambda, \mu)$ or $(\nu, \pi)$ face; or

(2) every $W$-face of $\Lambda$ is a $(\lambda, \mu)$ or $(\nu, \pi)$ face.

Proof. By, if necessary, reflecting the graph $G_{Q}$, interchanging vertices 1 and 2 of $G_{T}$, or reflecting $G_{T}$ (this reverses the order of the edge classes at vertices 1 and 2), we may assume that $\Lambda^{*}$ has an ordinary cycle with respect to $\omega$, at a vertex $x$, say, of the form shown in Figure 3.10. (We are using Lemma 3.3 here.) Note that this may switch the $B$ and $W$ sides.

On $G_{T}$, let $x$ denote the two endpoints, one at vertex 1 and one at vertex 2, corresponding to the West and South edges of $\Lambda$ at $x$, and let $x^{\prime}$ denote those corresponding to the East and North edges. Then these four endpoints appear on $G_{T}$ as in Figure 3.11.

Let $e_{2}$ be an edge of $G_{Q}$ in class $\lambda$ or $\mu$, with label 2 at a vertex $y$, say. Then, at vertex 2 of $G_{T}$, the label $y$ occurs between $x$ and $x^{\prime}$ (from left to 


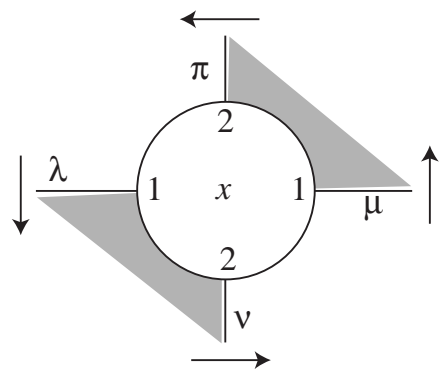

Figure 3.10 .

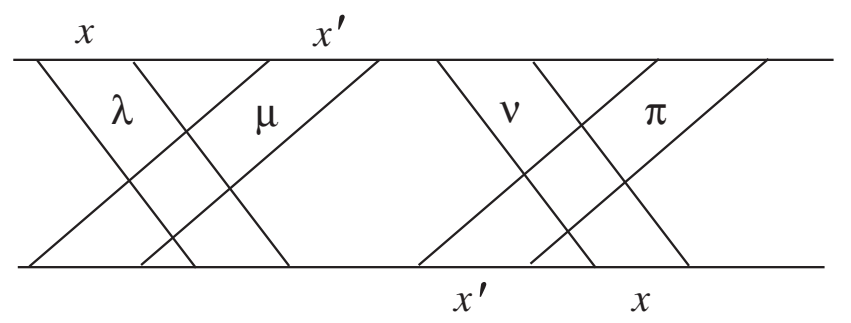

Figure 3.11.

right). By Lemma $3.2 y$ also appears between $x$ and $x^{\prime}$ at vertex 1 (from left to right). It follows that if the edge $e_{1}$ adjacent to $e_{2}$ on the $B$-side at $y$ is not a loop edge, then it belongs to class $\lambda$ or $\mu$. In particular, every $B$-face of $\Lambda$ containing an edge in class $\lambda$ or $\mu$ is a $(\lambda, \mu)$ face.

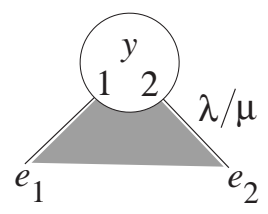

Figure 3.12.

A similar argument shows that every $B$-face of $\Lambda$ containing an edge in class $\nu$ or $\pi$ is a $(\nu, \pi)$ face.

Note. A statement completely analogous to Lemma 3.9 holds for the dual orientation $\omega^{\prime}$ (every face of some color in $\Lambda$ is a $(\pi, \lambda)$-face or a $(\mu, \nu)$-face).

A sink or source of $\omega, \omega^{\prime}$ in $\Lambda^{*}$ that does not correspond to the outside 
face of $\Lambda$ is a binary face of $\Lambda$. If this face is a bad binary face then we say the sink or source is bad. Note that we will not refer to a sink or source at the outside face as bad.

Lemma 3.10. If $\Lambda^{*}$ contains a bad sink or source with respect to $\omega$ or $\omega^{\prime}$, then $\Lambda^{*}$ does not contain an ordinary cycle with respect to any dual orientation.

Proof. Suppose, without loss of generality, that $\Lambda$ contains a bad $B(\lambda, \mu)$ face, corresponding to a sink or source of $\Lambda^{*}$ with respect to $\omega$. Then, by Lemma 3.5, without loss of generality, for any edge $e$ of $G_{Q}$ in class $\nu$ or $\pi$, the edges of $G_{Q}$ adjacent to $e$ on the $B$-side are in class $\lambda$.

Now let $v$ be a vertex of $\Lambda$ which corresponds to an ordinary cycle of $\Lambda^{*}$ with respect to any dual orientation. Then the four edges incident to $v$ are in distinct edge classes. But by the previous paragraph, each of the edges in classes $\nu$ and $\pi$ has adjacent to it on the $B$-side an edge in class $\lambda$. This is clearly impossible.

Lemma 3.11. Suppose that $\Lambda^{*}$ contains a sink or source with respect to $\omega$, and that all such sinks or sources are bad. Then $\Lambda^{*}$ does not contain two exceptional cycles with respect to $\omega^{\prime}$ or $\omega^{\prime \prime}$.

Proof. Without loss of generality, we may suppose that $\Lambda$ contains a bad $B(\lambda, \mu)$ face, and that $\varepsilon=\lambda$ in the conclusion of Lemma 3.5.

Recall that if $f$ is a bad $B(\lambda, \mu)$ face, $v_{\lambda}(f)$ denotes some vertex of $f$ at which $f$ has a $B(\lambda, \lambda)$ corner.

Claim. Let $f$ be a $B(\lambda, \mu)$ face of $\Lambda$. Then either $v_{\lambda}(f)$ is exceptional, or the face of $\Lambda^{*}$ dual to $v_{\lambda}(f)$ has index -1 with respect to $\omega$.

Proof. If $v_{\lambda}(f)$ is not exceptional, then the other two edges at $v_{\lambda}(f)$ must be in class $\mu$, by Lemma 3.5. (Note that neither can be in class $\lambda$ by Lemma 3.3.) Hence the corresponding dual face of $\Lambda^{*}$ has index -1 with respect to $\omega$.

By Lemma 3.6, the sinks and sources of $\Lambda^{*}$ with respect to $\omega$ correspond to either

(1) $B(\lambda, \mu)$ and $W(\lambda, \mu)$ faces of $\Lambda$; or

(2) $B(\lambda, \mu)$ and $W(\nu, \pi)$ faces of $\Lambda$. 
Let $\mathcal{B}(\lambda, \mu)$ denote the set of $B(\lambda, \mu)$ faces of $\Lambda, \mathcal{W}(\lambda, \mu)$ the set of $W(\lambda, \mu)$ faces of $\Lambda$, etc..

First consider case (1). If $\mathcal{W}(\lambda, \mu) \neq \emptyset$, then by Lemma 3.5 we have either

$\left(\mathrm{a}^{\prime}\right)$ the edges of $G_{Q}$ adjacent on the $W$-side to any edge of $G_{Q}$ in class $\nu$ or $\pi$ are in class $\lambda$; or

$\left(\mathrm{b}^{\prime}\right)$ these edges are in class $\mu$.

Suppose $\left(\mathrm{a}^{\prime}\right)$ holds. Then the Claim above is also true for $f \in \mathcal{W}(\lambda, \mu)$. Let $V$ denote the set of vertices of $\Lambda$, and define

$$
\varphi: \mathcal{B}(\lambda, \mu) \cup \mathcal{W}(\lambda, \mu) \rightarrow V
$$

by setting $\varphi=v_{\lambda}(f)$.

Clearly $\varphi$ is one-one (by Lemma 3.3).

Let $e_{\lambda}^{B}, e_{\lambda}^{W}$ be the number of exceptional vertices in $v_{\lambda}(\mathcal{B}(\lambda, \mu))$ and $v_{\lambda}(\mathcal{W}(\lambda, \mu))$ respectively. Then by the Claim,

$$
t \geq s-\left(e_{\lambda}^{B}+e_{\lambda}^{W}\right)
$$

Hence, by Lemma 3.8(1)

$$
s+c+c_{e}+d \geq 2+s-\left(e_{\lambda}^{B}+e_{\lambda}^{W}\right) .
$$

Also, $c=0$, by Lemma 3.10. Therefore

$$
c_{e}+e_{\lambda}^{B}+e_{\lambda}^{W} \geq 2-d .
$$

On the other hand, we clearly have

$$
c_{e}+e_{\lambda}^{B}+e_{\lambda}^{W} \leq 2 .
$$

(There are at most 2 exceptional vertices and any such cannot contribute to both $c_{e}$ and $e_{\lambda}^{B}$ or $e_{\lambda}^{W}$.) It follows that $d=0$ or 1 .

If $d=0$, then

$$
c_{e}+e_{\lambda}^{B}+e_{\lambda}^{W}=2 .
$$

Thus there are two exceptional vertices, and each either corresponds to a cycle of $\Lambda^{*}$ with respect to $\omega$, or has incident to it two edges in class $\lambda$. It follows that neither corresponds to an exceptional cycle with respect to $\omega^{\prime}$ or $\omega^{\prime \prime}$. 
If $d=1$, then $c_{e}=0$, by Lemma 3.8(2), hence $e_{\lambda}^{B}+e_{\lambda}^{W}=1$ or 2 . Thus at least one exceptional vertex has incident to it two edges in class $\lambda$, and so there cannot be two exceptional cycles with respect to $\omega^{\prime}$ or $\omega^{\prime \prime}$.

This completes the proof in case $\left(\mathrm{a}^{\prime}\right)$.

If $\left(b^{\prime}\right)$ holds, define

$$
\varphi: \mathcal{B}(\lambda, \mu) \cup \mathcal{W}(\lambda, \mu) \rightarrow V
$$

by $\varphi\left|\mathcal{B}(\lambda, \mu)=v_{\lambda}, \varphi\right| \mathcal{W}(\lambda, \mu)=v_{\mu}$. The argument is then completely analogous to case $\left(\mathrm{a}^{\prime}\right)$.

If $\mathcal{W}(\lambda, \mu)=\emptyset$, follow the argument of Case $\left(1 \mathrm{a}^{\prime}\right)$ above with $e_{\lambda}^{W}=0$.

Finally, consider case (2). Here, if $\mathcal{W}(\nu, \pi) \neq \emptyset$, we have either

$\left(\mathrm{a}^{\prime \prime}\right)$ the edges of $G_{Q}$ adjacent on the $W$-side to any edge of $G_{Q}$ in class $\lambda$ or $\mu$ are in class $\nu$; or

$\left(\mathrm{b}^{\prime \prime}\right)$ these edges are in class $\pi$.

Define $\varphi: \mathcal{B}(\lambda, \mu) \cup \mathcal{W}(\nu, \pi) \rightarrow V$ by $\varphi \mid \mathcal{B}(\lambda, \mu)=v_{\lambda}$, and $\varphi \mid \mathcal{W}(\nu, \pi)=v_{\nu}$ or $v_{\pi}$, according to whether $\left(\mathrm{a}^{\prime \prime}\right)$ or $\left(\mathrm{b}^{\prime \prime}\right)$ holds.

The argument is now exactly as in case (1).

Lemma 3.12. Suppose that $\Lambda^{*}$ contains a bad sink or source with respect to $\omega$.

(1) If all sinks and sources of $\Lambda^{*}$ with respect to $\omega^{\prime}$ are bad, then $\Lambda^{*}$ does not contain a sink or source with respect to $\omega^{\prime}$.

(2) If all sinks and sources of $\Lambda^{*}$ with respect to $\omega^{\prime \prime}$ are bad, then $\Lambda^{*}$ does not contain a sink or source with respect to $\omega^{\prime \prime}$.

Proof. Suppose that $\Lambda^{*}$ contains a bad sink or source with respect to $\omega$. Then, without loss of generality, $\Lambda$ contains a bad $B(\lambda, \mu)$ face. By Lemma 3.5, we then have either

(a) the edges of $G_{Q}$ adjacent on the B-side to any edge of $G_{Q}$ in class $\nu$ or $\pi$ are in class $\lambda$;

or

(b) these edges are in class $\mu$. 
(1) Suppose that $\Lambda^{*}$ also contains a bad sink or source with respect to $\omega^{\prime}$. By Lemma 3.6, the corresponding dual face of $\Lambda$ is a $W$-face; without loss of generality it is a $W(\pi, \lambda)$ face.

Suppose (b) holds. Since $\Lambda$ contains a bad $W(\pi, \lambda)$ face, there is a $W(\pi, \pi)$ corner at some vertex $v$ of $\Lambda$. Then (b) implies that the other two edges at $v$ are in class $\mu$. Thus $G_{Q}$ contains $W(\lambda, \lambda),(\pi, \pi)$ and $(\mu, \mu)$ corners, contradicting Lemma 3.4.

Therefore (a) holds.

Let $f$ be a face of $\Lambda$ corresponding to a sink or source of $\Lambda^{*}$ with respect to $\omega^{\prime}$. By Lemma 3.6, $f$ is a bad $W(\pi, \lambda)$ face. Recall that $v_{\pi}(f)$ denotes some vertex in the boundary of $f$ at which $f$ has a $W(\pi, \pi)$ corner. Then, by (a), the other two edges of $G_{Q}$ at $v_{\pi}(f)$ are in class $\lambda$. Hence the face of $\Lambda^{*}$ dual to $v_{\pi}(f)$ has index -1 with respect to $\omega^{\prime}$. Note also that $f \neq f^{\prime}$ implies $v_{\pi}(f) \neq v_{\pi}\left(f^{\prime}\right)$. Therefore, using $s^{\prime}$, etc. to denote the number of sinks and sources, etc. with respect to $\omega^{\prime}$, we have

$$
t^{\prime} \geq s^{\prime}
$$

Hence, by Lemma 3.8(1),

$$
s^{\prime}+c^{\prime}+c_{e}^{\prime}+d^{\prime} \geq 2+t^{\prime} \geq 2+s^{\prime} .
$$

Also, $c^{\prime}=0$ by Lemma 3.10. Hence

$$
c_{e}^{\prime}+d^{\prime} \geq 2 .
$$

It follows, by Lemma 3.8(3), that $c_{e}^{\prime}=2$. But this contradicts Lemma 3.11.

(2) Suppose that $\Lambda^{*}$ contains a bad sink or source with respect to $\omega^{\prime \prime}$. By Lemma 3.6 we may assume that any face of $\Lambda$ dual to a sink or source of $\Lambda^{*}$ with respect to $\omega^{\prime \prime}$ is a bad $W(\lambda, \nu)$ face. The rest of the argument is then exactly as in (1) above, with $\pi$ replaced by $\nu$.

Lemma 3.13. If $\Lambda^{*}$ contains two oppositely oriented exceptional cycles with respect to $\omega$, then $\Lambda^{*}$ does not contain a bad sink or source with respect to $\omega^{\prime \prime}$.

Proof. Suppose that $\Lambda^{*}$ contains two oppositely oriented exceptional cycles with respect to $\omega$. Since $T$ is separating there must be an exceptional endpoint of $\Lambda$ labelled 1 and another labelled 2. Let $x$ (resp. $y$ ) be the exceptional vertex whose exceptional endpoint has label 1 (resp. 2). 
By reflecting $G_{Q}$ if necessary, we may assume that the cycle at $x$ is clockwise. Also, by reflecting $G_{T}$ if necessary (i.e. reversing the cyclic order of the edge classes $\lambda, \mu, \nu, \pi$ at the vertices of $G_{T}$ ), we may assume that the edge of $\Lambda^{*}$ in the cycle at $x$ directed into the outside region is dual to an edge of $\Lambda$ in class $\mu$. The edge of $\Lambda$ with label 1 at $x$ is then in either class $\nu$ or $\pi$. We therefore have the two possibilities $C 1$ and $C 2$ shown in Figure 3.13.

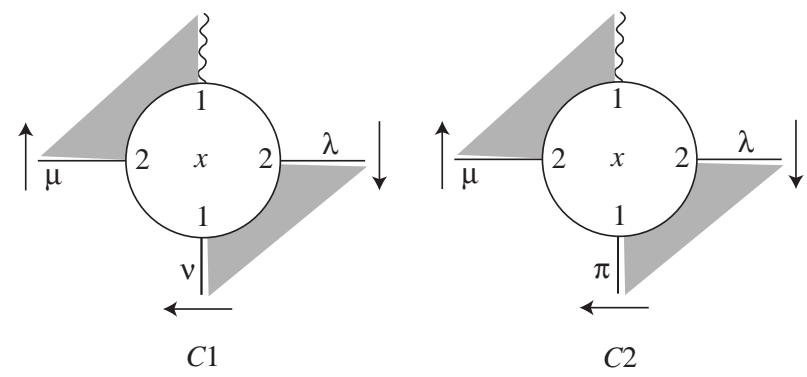

Figure 3.13 .

There are four possibilities for the anticlockwise cycle at $y$, shown in Figure 3.14.

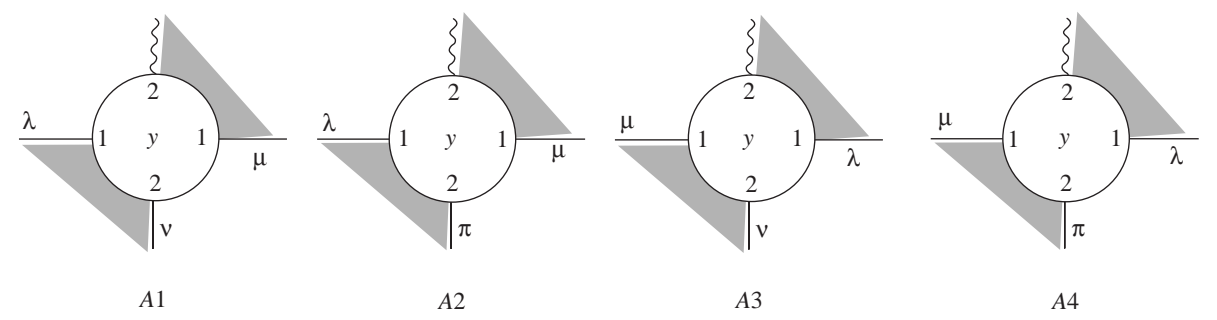

Figure 3.14.

So, a priori, we have eight cases to consider. However, there are further symmetries. Thus, interchanging the labels 1 and 2 interchanges $C 1$ and $A 3$, and $C 2$ and $A 4$, while interchanging labels 1 and 2 and reversing the order of the edge classes $\lambda, \mu, \nu, \pi$ (i.e. reflecting $G_{T}$ ) interchanges $C 1$ and $A 2$, and $C 2$ and $A 1$. It is therefore enough to consider the six possibilities $(C 1, A 1)$, $(C 1, A 2),(C 1, A 3),(C 1, A 4),(C 2, A 1)$, and $(C 2, A 4)$. We shall see below that three of these are actually impossible.

To analyze the edges incident to $x$ and $y$ as they lie on the graph $G_{T}$, note that at $x$ and $y$ there are two edges of $\Lambda$ that are separated by a $B$ corner. We will label the two corresponding endpoints at vertices 1 and 2 of 
$G_{T}$ by $x$ and $y$, respectively. The third edge of $\Lambda$ at $x$ (resp. $y$ ), with label 2 (resp. 1), will be labelled at vertex 2 (resp. 1) of $G_{T}$ by $x^{\prime}$ (resp. $y^{\prime}$ ).

First observe that cases $(C 1, A 3),(C 1, A 4)$, and $(C 2, A 4)$ are impossible. For example, consider $(C 1, A 3)$. Then the labels $x, x^{\prime}, y, y^{\prime}$ corresponding to the endpoints of the edges of $\Lambda$ at $x$ and $y$ appear at vertices 1 and 2 of $G_{T}$ as shown in Figure 3.15. Label the endpoints of the ghost edge at $x$ (resp. $y$ ) by $x^{\prime}$ (resp. $\left.y^{\prime}\right)$. Since $x^{\prime}$ must lie between $y^{\prime}$ and $y$ at vertex 1 , and $y^{\prime}$ must lie between $x^{\prime}$ and $x$ at vertex 2 , the orderings of the labels $x, x^{\prime}, y, y^{\prime}$ at vertices 1 and 2 violate Lemma 3.2. Similarly, one sees that cases $(C 1, A 4)$ and $(C 2, A 4)$ are also impossible.

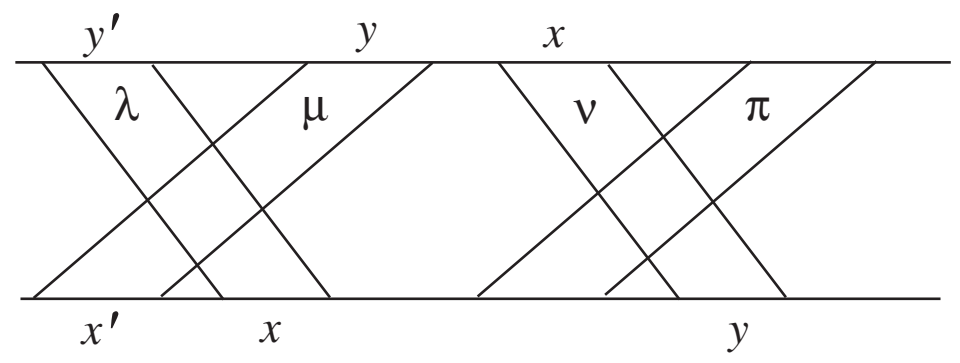

Figure 3.15.

Hence we are left with the cases $(C 1, A 1),(C 1, A 2)$ and $(C 2, A 1)$. The labels $x, x^{\prime}, y, y^{\prime}$ at vertices 1 and 2 of $G_{T}$ in these three cases are shown in Figure 3.16.

To facilitate the subsequent arguments we make the following convention. If $z$ is a vertex of $G_{Q}$ then $z$ appears twice as a label of vertex 1 of $G_{T}$ and twice as a label of vertex 2. On vertex 1 we call one of these labels $z$ and the other $z^{\prime}$. On vertex 2 we do the same. We will do this in such a way that the labels $z$ in vertices 1 and 2 (of $G_{T}$ ) correspond to endpoints on vertex $z$ (of $G_{Q}$ ) that cobound a $B$ corner of $z$. The labels $z^{\prime}$ on vertices 1 and 2 will also cobound a $B$ corner. The label pairs $z, z^{\prime}$ on vertices 1 and 2 will cobound $W$ corners at vertex $z$ (of $G_{Q}$ ). With this labelling scheme, Lemma 3.2 says that the anti-clockwise cyclic ordering of a set of labels on vertex 1 of $G_{T}$ will be the same as the clockwise ordering of those labels on vertex 2 respecting the primes. It also says that the anti-clockwise cyclic ordering of the labels on vertex 1 will be the same as the clockwise cyclic ordering of the labels on vertex 2 when primed labels on 1 are compared to unprimed labels on 2 and unprimed labels on 1 compared to primed on 2 . We also note that moving from left to right in a diagram like Figure 2.2 is 
anti-clockwise on vertex 1 and clockwise on vertex 2 .

Now suppose that $\Lambda^{*}$ contains a bad sink or source with respect to $\omega^{\prime \prime}$. This corresponds to a bad face of $\Lambda$ that is either a $B(\lambda, \nu), W(\lambda, \nu), B(\mu, \pi)$ or $W(\mu, \pi)$ face. We shall show that each of these leads to a contradiction.

Suppose, for example, that we are in case $(C 1, A 1)$ and that $\Lambda$ contains a bad $B(\lambda, \nu)$ face. Such a face contains a $B(\lambda, \lambda)$ corner and a $B(\nu, \nu)$ corner, at vertices $u$ and $v$, say. Consider the two corresponding occurrences of the label $u$ (resp. $v$ ) at vertices 1 and 2 of $G_{T}$. First note that, at vertex $1, v$ (in class $\nu$ ) must occur to the right of $x$, otherwise it would lie between $x^{\prime}$ and $x$ at vertex 1 , and hence between $x^{\prime}$ and $x$ at vertex 2 , contradicting the fact that $v$ is also in class $\nu$ at vertex 2. Therefore the labels $x, v, y$ occur in that order at vertex 1 , hence at vertex 2 , and so $v$ occurs to the left of $y$ (in class $\nu$ ) at vertex 2. Now consider the label $u$, which lies in class $\lambda$ at vertices 1 and 2 . If it lies to the right of $y$ at vertex 1 , then it lies between $y$ and $x^{\prime}$ (since $x^{\prime}$ is extremal wrt $\Lambda$, by Theorem 2.1), and hence cannot lie in class $\lambda$ at vertex 2 . On the other hand, if it lies to the left of $y$ at vertex 1 , then the labels $v, u, y$ occur in that order at vertex 1 , hence at vertex 2 by Lemma 3.2, contradicting the fact that $u$ is in class $\lambda$ at vertex 2 .

Next, suppose that we are in either case $(C 1, A 1)$ or $(C 1, A 2)$, and that $\Lambda$ contains a $W(\pi, \pi)$ corner, at a vertex $w$, say. Then the label $w$ at vertex 1 of $G_{T}$ appears between $x$ and $y$, and hence $w^{\prime}$ appears between $x^{\prime}$ and $y^{\prime}$ at vertex 2 by Lemma 3.2. But this contradicts the fact that $w^{\prime}$ must lie in class $\pi$ at vertex 2 . Hence, in particular, $\Lambda$ cannot contain a bad $W(\mu, \pi)$ face.

A similar argument shows that in case $(C 2, A 1)$ we cannot have a $B(\nu, \nu)$ corner, and hence $\Lambda$ cannot contain a bad $B(\lambda, \nu)$ face.

Similar arguments show that in case $(C 1, A 2), \Lambda$ cannot contain a $B(\nu, \nu)$ corner, and in case $(C 2, A 1), \Lambda$ cannot contain a $W(\pi, \pi)$ corner.

Finally, suppose we are in case $(C 1, A 1)$, and that $\Lambda$ contains a $B(\mu, \pi)$ corner, at a vertex $z$, say. Then the label $z$ at vertex 1 of $G_{T}$ is in class $\mu$, and so lies between $x^{\prime}$ and $x$ (since $x^{\prime}$ at 1 is extremal, by Theorem 2.1). Hence $z$ lies between $x^{\prime}$ and $x$ at vertex 2, contradicting the fact that $z$ is in class $\pi$ at vertex 2 . Essentially the same argument shows that $\Lambda$ cannot contain a $W(\lambda, \nu)$ corner. Thus in the presence of $(C 1, A 1)$ there is neither a bad $B(\mu, \pi)$ face nor $W(\lambda, \nu)$ face. Together these arguments show that in case $(C 1, A 1), \Lambda^{*}$ contains no bad sink or source with respect to $w^{\prime \prime}$.

Similar arguments show that in cases $(C 1, A 2)$ and $(C 2, A 1), \Lambda$ cannot contain a $B(\mu, \pi)$ corner, or a $W(\lambda, \nu)$ corner; consequently, no bad $B(\mu, \pi)$ on $W(\lambda, \nu)$ face. With the above arguments, this rules out a bad sink or source with respect to $\omega^{\prime \prime}$ in cases $(C 1, A 2)$ and $(C 2, A 1)$. 


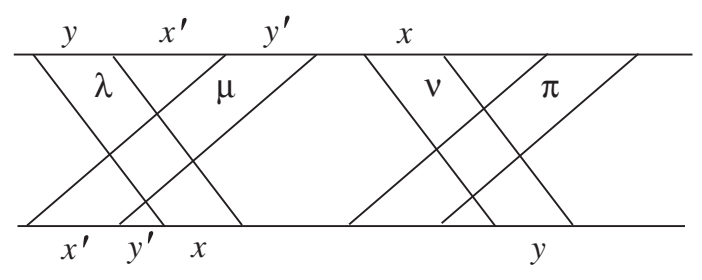

$(C 1, A 1)$

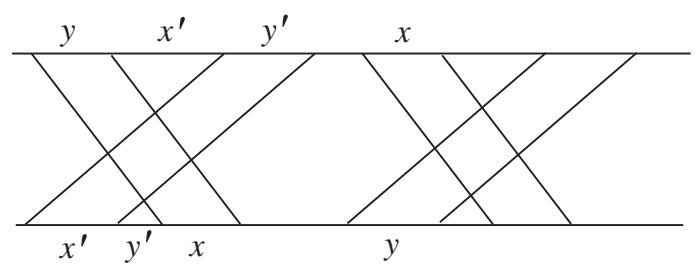

$(C 1, A 2)$

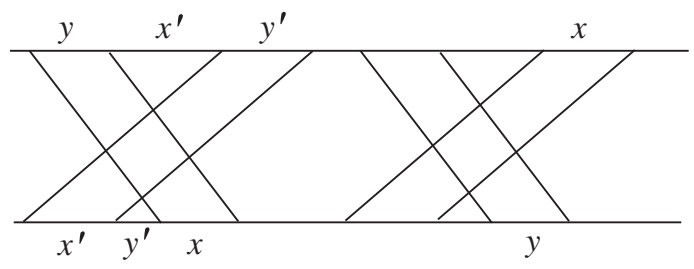

$(C 2, A 1)$

Figure 3.16.

Lemma 3.14. If $\Lambda^{*}$ contains two oppositely oriented exceptional cycles with respect to $\omega$, then $\Lambda^{*}$ does not contain an ordinary cycle with respect to $\omega^{\prime \prime}$.

Proof. As in the proof of Lemma 3.13, there are, up to symmetry, three possibilities for the two oppositely oriented exceptional cycles with respect to $\omega,(C 1, A 1),(C 1, A 2)$ and $(C 2, A 1)$. The corresponding labels as they appear on $G_{T}$ are illustrated in Figure 3.16.

Suppose $\Lambda^{*}$ contains an ordinary cycle with respect to $\omega^{\prime \prime}$, at a vertex $u$, say. There are four possibilities for such a cycle, shown in Figure 3.17.

To indicate the four endpoints at $u$ as they appear on $G_{T}$, we shall use $u$ to denote the two endpoints, one at vertex 1 and one at vertex 2 , corresponding to the West and South edges of $\Lambda$ at $u$, and $u^{\prime}$ to denote those corresponding to the East and North edges. 
The proof proceeds by showing that in all cases, the orderings of the labels $x, x^{\prime}, y, y^{\prime}, u, u^{\prime}$ at vertices 1 and 2 of $G_{T}$ are inconsistent. We do two representative cases; the other cases are analogous.

Suppose $(C 1, A 1)$ and $O 1$ hold. Then $u$ is in class $\lambda$ at vertex 1 and hence lies between $x$ and $x^{\prime}$ as you go from left to right (since $x^{\prime}$ at vertex 1 is extremal, by Theorem 2.1), and hence at vertex $2 u^{\prime}$ lies between $x^{\prime}$ and $x$ as you go from left to right. But this contradicts the fact that $u^{\prime}$ is in class $\pi$ at vertex 2 .

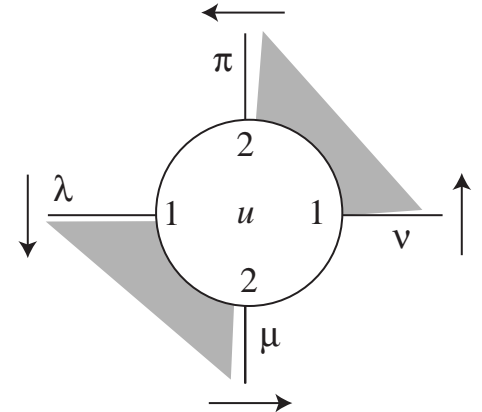

$O 1$

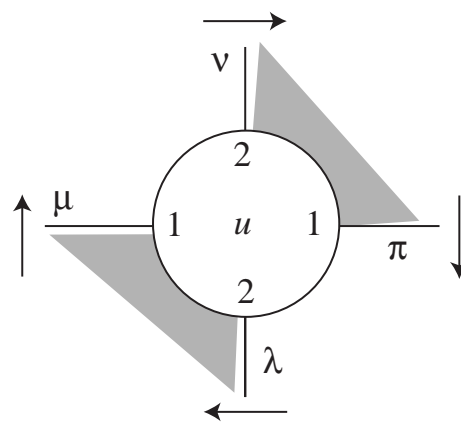

O3

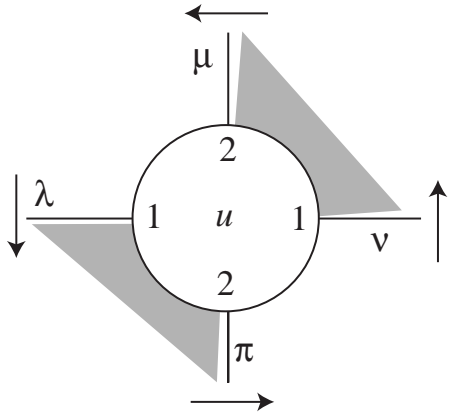

$\mathrm{O} 2$

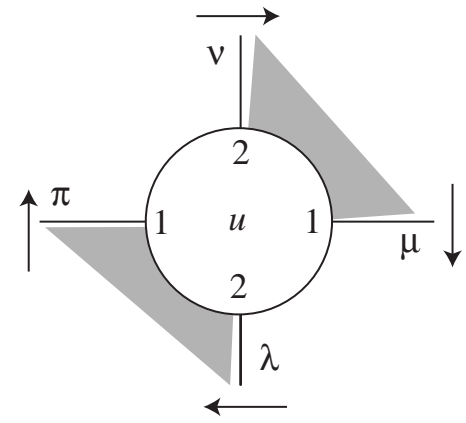

O4

Figure 3.17.

Suppose $(C 1, A 2)$ and $O 3$ hold. Then $u^{\prime}$ at vertex 1 is in class $\pi$, and hence lies between $x$ and $y$ (from left to right). Therefore $u$ at vertex 2 lies between $x^{\prime}$ and $y^{\prime}$ (from left to right). But since $y^{\prime}$ at vertex 2 is extremal, by Theorem 2.1, this contradicts the fact that $u$ is in class $\lambda$ at vertex 2 .

Similar arguments apply to all the other cases. 
Proof of Theorem 3.1. By Lemma 8.2 of [15], $\Lambda$ contains a face of length 2 or $3, g$, which we may assume is a $B$-face. Note that $g$ is necessarily a good binary face.

In the argument below, the number of sinks and sources of $\Lambda^{*}$, etc., with respect to $\omega, \omega^{\prime}$ and $\omega^{\prime \prime}$, will be denoted by $s, s^{\prime}, s^{\prime \prime}$, etc. .

There are two cases

(I) The edge classes of $g$ are adjacent.

(II) The edge classes of $g$ are non-adjacent.

(I) We may assume without loss of generality that $g$ is a $B(\mu, \nu)$ face.

We will consider the two dual orientations $\omega$ and $\omega^{\prime \prime}$. By Lemma 3.7, neither $\omega$ nor $\omega^{\prime \prime}$ has a sink or source corresponding to a good $B$-face. If $\omega$ or $\omega^{\prime \prime}$ has a sink or source corresponding to a good $W$-face $f$, then $f$ has exactly one edge class in common with $g$, and Theorem 3.1 is proved. So we suppose that any face of $\Lambda$ corresponding to a sink or source of $\Lambda^{*}$ with respect to $\omega$ or $\omega^{\prime \prime}$ is bad, and show that this leads to a contradiction.

We distinguish two subcases.

Subcase (A). $\Lambda^{*}$ contains two oppositely oriented exceptional cycles with respect to $\omega$.

Then, by Lemma 3.13, $s^{\prime \prime}=0$, and by Lemma 3.14, $c^{\prime \prime}=0$. Hence by Lemma $3.8(4), c_{e}^{\prime \prime}=2$. But since no exceptional vertex can be dual to a cycle of $\Lambda^{*}$ with respect to both $\omega$ and $\omega^{\prime \prime}$, this contradicts our hypothesis.

Subcase (B). $\Lambda^{*}$ does not contain two oppositely oriented exceptional cycles with respect to $\omega$.

Then, by Lemma 3.8(4), $s+c>0$.

First suppose $c>0$. Then either (1) or (2) of Lemma 3.9 holds. Since $g$ is a $B(\mu, \nu)$ face, (1) is impossible. On the other hand, (2) implies that every $W$-face is a (bad) sink or source with respect to $\omega$. Since $\Lambda$ definitely contains a $W$-face, this contradicts Lemma 3.10.

Hence $s>0$. Therefore we have $c^{\prime \prime}=0$, by Lemma 3.10, and $s^{\prime \prime}=0$, by Lemma $3.12(2)$. Hence $c_{e}^{\prime \prime}=2$ by Lemma 3.8(4). But this contradicts Lemma 3.11.

(II) We may assume without loss of generality that $g$ is a $B(\lambda, \nu)$ face.

We will consider the dual orientations $\omega$ and $\omega^{\prime}$. If either $\omega$ or $\omega^{\prime}$ has a sink or source corresponding to a good face $f$, then $f$ is a $W$-face, by Lemma 3.7, and shares exactly one edge class with $g$. So we suppose that any face of $\Lambda$ corresponding to a sink or source of $\Lambda^{*}$ with respect to $\omega$ or $\omega^{\prime}$ is bad, and show that this leads to a contradiction. 
As in subcase (B) of case (I) above, Lemmas 3.9 and 3.10 imply that $c=0$. Similarly (using Lemma 3.9 with $\omega$ replaced by $\omega^{\prime}$ ), $c^{\prime}=0$.

By Lemma 3.12(1), either $s=0$ or $s^{\prime}=0$; without loss of generality, assume $s^{\prime}=0$. Then, by Lemma 3.8(4), we have $c_{e}^{\prime}=2$. Hence, by Lemma 3.11, $s=0$ also. By Lemma 3.8(4) again, $c_{e}=2$. Since no exceptional vertex (with only one ghost label) can be dual to a cycle of $\Lambda^{*}$ with respect to both $\omega$ and $\omega^{\prime}$, this is a contradiction.

\section{Using good binary faces.}

In this section we show that the existence of a good face of $\Lambda$ implies that the side of $\widehat{T}$ in $K(p / 2)$ in which the face lies is a Seifert fiber space over the disk with two exceptional fibers.

Theorem 4.1. Assume that $\Lambda$ contains a good $X(\varepsilon, \delta)$ face of length $n$. Then the $X$ side of $\widehat{T}$ in $K(p / 2)$ is a Seifert fiber space over the disk with exactly two exceptional fibers, one of which has order $n$. Moreover the Seifert fiber of this fibration is the curve on $\widehat{T}$ formed by $\varepsilon \cup \delta$.

Let $f$ be an $\varepsilon$-good $(\varepsilon, \delta)$ face. Consider the edges of $G_{T}$ corresponding to the edges in $\partial f$. The endpoints of these edges at vertex $j$ of $G_{T}, j=1,2$, give a set of labels, corresponding to the vertices of $\partial f$. If we read these labels anticlockwise around vertex 1, then the same labels appear in clockwise order around vertex 2 . For convenience, number these labels $0,1, \ldots, n-1$ in anticlockwise order around vertex 1.

Let $\left[a_{0}, a_{1}\right]$ be the (cyclic) interval of labels corresponding to the endpoints at vertex 1 of the edges of $\partial f$ that are in class $\delta$. Similarly, let $\left[b_{0}, b_{1}\right]$ be the interval of labels corresponding to the endpoints at vertex 2 of the edges that are in class $\varepsilon$. See Figure 4.1.

Lemma 4.2. Either $b_{0}=a_{0}$ or $b_{1}=a_{1}$.

Proof. Since $f$ is $\varepsilon$-good, as we read around $\partial f$, each edge in class $\varepsilon$ is immediately followed by an edge in class $\delta$. Therefore $\left[b_{0}, b_{1}\right] \subset\left[a_{0}, a_{1}\right]$.

Suppose $b_{0} \neq a_{0}$ and $b_{1} \neq a_{1}$. Then $\left[b_{0}-1, b_{1}+1\right] \subset\left[a_{0}, a_{1}\right]$.

Now let $n_{\delta}$ be the total number of edges of $G_{T}$ in class $\delta$, and let $p$ be the total number of labels of endpoints of edges of $G_{T}$ (with multiplicity) in the interval $\left[b_{0}-1, b_{1}+1\right]$. Then, looking at vertex 2 we see that

$$
p+n_{\delta}>2 q \text {. }
$$




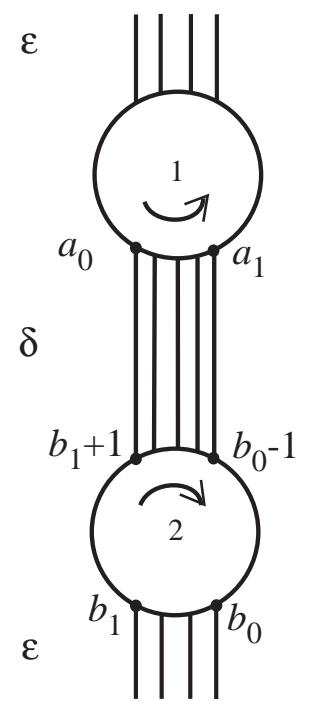

Figure 4.1.

On the other hand, since $\left[b_{0}-1, b_{1}+1\right] \subset\left[a_{0}, a_{1}\right]$, by considering vertex 1 we have

$$
p \leq n_{\delta} .
$$

Hence $n_{\delta}>q$. The construction of [11] then gives the contradiction that $K$ is a cable knot.

We restate Lemma 4.2 as follows. Let $f$ be a good $(\varepsilon, \delta)$-face of $\Lambda$. There are vertices $x, y$ of $f$ in $\Lambda$, representing corners of $\partial f$, such that the edges of $\partial f$ on $G_{T}$ are, up to homeomorphism, as in Figure 4.2. The interval $[x, y]$ on vertex $i$ of $G_{T}, i=1,2$, that contains no edges of $\partial f$ in its interior, is called the external interval of $f$ on vertex $i$ and is denoted $I_{f}^{i}$. (If $f$ is a bigon there are two such intervals.)

Lemma 4.3. Let $X$ be a genus 2 handlebody, and let $F \subset \partial X$ be a oncepunctured torus such that inclusion induces an isomorphism $\pi_{1}(F) \rightarrow \pi_{1}(X)$. Let $\gamma$ be a non-separating simple closed curve in $\partial X-F$. Then the manifold obtained by attaching a 2-handle to $X$ along $\gamma$ is a solid torus.

Proof. Let $Y$ be the result of attaching a 2-handle to $X$ along $\gamma$. Then $\partial Y$ is a torus containing $F$. Since $\pi_{1}(F) \rightarrow \pi_{1}(X)$ is onto, $\pi_{1}(\partial Y) \rightarrow \pi_{1}(Y)$ is onto. Therefore, by the Loop Theorem and Dehn's Lemma, $Y \cong S^{1} \times D^{2} \# M$, 


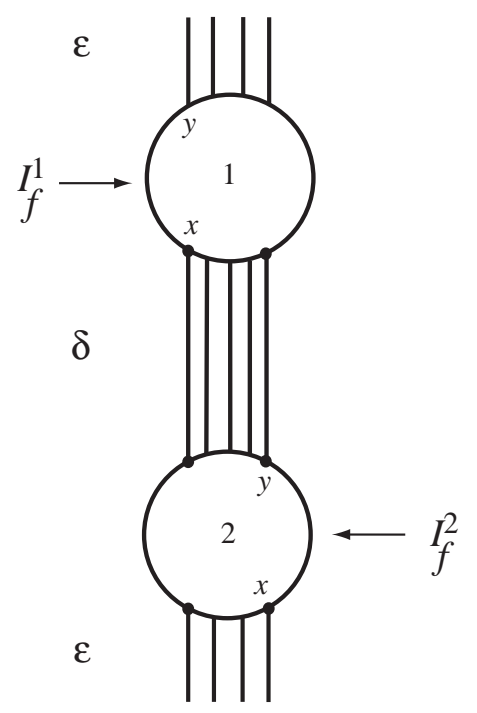

Figure 4.2.

where $M$ is a homotopy 3 -sphere. Also, $M$ has Heegaard genus at most 1 by [18]. Hence $M \cong S^{3}$ and $Y \cong S^{1} \times D^{2}$.

Let $K(p / 2)=M_{B} \cup_{\widehat{T}} M_{W}$, where $M_{B}$ and $M_{W}$ are the $B$ and $W$ sides of $\widehat{T}$, respectively.

Without loss of generality assume that the face in the hypothesis of Theorem 4.1 is a good $B(\varepsilon, \delta)$ face $f$ of length $n$. Note that $T$ is incompressible in $E(K)$, thus we may assume that $f$ lies entirely in $M_{B}$. Let $V$ be the solid torus in the Dehn surgery $K(p / 2)=E(K) \cup V$. Let $H=V \cap M_{B}$. Let $A$ be an annulus on $\widehat{T}$ such that $\partial f \subset A \cup H$ (think of $f$ now as a properly embedded disk in the $B$ side of $T$ in $E(K))$. Note that the circuit $\varepsilon \cup \delta$ in $G_{T}$ gives a core curve for $A$ in $\widehat{T}$.

Lemma 4.4. Let $W$ be a regular neighborhood of $A \cup H \cup f$ in $M_{B}$. Then $W$ is a solid torus, in which the core of $A$ is homotopic to $n$ times the core of $W$.

Proof. Identifying $A$ with $A \times\{1\}$ in $A \times I, W$ is homeomorphic to the manifold obtained by attaching a 2-handle to the genus 2 handlebody $A \times$ $I \cup H$ along the curve $\partial f$. Under this homeomorphism, $A \subset \partial W$ corresponds to $A \times\{0\}$. 
Let $I_{f}^{j}$ be the external interval associated with $f$ at vertex $j$, and let $x^{j} \in \operatorname{Int} I_{f}^{j}, j=1,2$. There is an arc $\xi \subset \partial H$ running from $x^{1}$ to $x^{2}$, disjoint from $\partial f$. There are also disjoint arcs $\gamma^{1}$ and $\gamma^{2}$ in $\overline{A-H}$, which are disjoint from $\partial f$, running from $x^{1}$ and $x^{2}$ respectively to (distinct components of) $\partial A \times\{1\}$. Let $b$ be a regular neighborhood of $\gamma^{1} \cup \xi \cup \gamma^{2}$ in $\overline{A-H} \cup \partial H$. Finally, let $F=A \times\{0\} \cup \partial A \times I \cup b$. Then $F$ is a once-punctured torus, and clearly $\pi_{1}(F) \rightarrow \pi_{1}(A \times I \cup H)$ is onto. Since $\partial f \cap F=\emptyset, W$ is a solid torus by Lemma 4.3.

On the other hand, note that if we attach a 2-handle to $W$ along $A$, we get a punctured lens space whose fundamental group has order $n$. This implies that the core of $A$ is homotopic to $n$ times the core of $W$.

Remark. Lemma 4.4 implies that if $f$ is $\varepsilon$-good and not a bigon, then there are more $\delta$-edges in $\partial f$ than $\varepsilon$-edges. Otherwise $\partial f$ would alternate in $\varepsilon$ and $\delta$-edges and $H_{1}(W)$ would have non-trivial torsion.

Proof of Theorem 4.1. Let $\widehat{T}^{\prime}=(\widehat{T}-\operatorname{Int} A) \cup(\partial W-\operatorname{Int} A)$. Then $\widehat{T}^{\prime} \subset$ $K(p / 2)$ is a 2 -torus which misses $K_{p / 2}$. That is, $\widehat{T}^{\prime} \subset E(K)$. Now $\widehat{T}^{\prime}$ is not isotopic to $\partial E(K)$, since the side of $\widehat{T}^{\prime}$ containing $\partial E(K)$ contains the essential torus $\widehat{T}$. By hypothesis then, $\widehat{T}^{\prime}$ must be compressible in $E(K)$, hence in $K(p / 2)$. But one side of $\widehat{T}^{\prime}$ in $K(p / 2)$ is $W \cup_{A} M_{W}$. Thus it must be that $\widehat{T}^{\prime}$ compresses on its other side, $W^{\prime}=\overline{M_{B}-W}$. The irreducibility of $E(K)$ implies that $W^{\prime}$ is a solid torus. Thus if $A^{\prime}$ is the annulus $\overline{\partial W-A}$, we have written $M_{B}$ as the union of two solid tori along an annulus $A^{\prime}$ : $M_{B}=W \cup_{A^{\prime}} W^{\prime}$. Since $\widehat{T}$ does not compress in $M_{B}, M_{B}$ is a Seifert fiber space with exactly two exceptional fibers. Since the core of $A^{\prime}$ is isotopic in $\partial W$ to the core of $A$, one of these exceptional fibers has order $n=\operatorname{length}(f)$.

\section{A link surgery description of $K$.}

In this section, the subscript $i$ will always denote 1 or 2 . As given by Theorem 3.1, let $f_{i}$ be a good $X_{i}\left(\varepsilon, \delta_{i}\right)$ face of $\Lambda$, where $\left\{X_{1}, X_{2}\right\}=\{B, W\}$. Denote by $I_{i}^{j}$ the external interval $I_{f_{i}}^{j}$ of $f_{i}$ on vertex $j$ of $G_{T}, j=1,2$. (See the definition after Lemma 4.2.) In particular, let $I_{i}$ stand for either $I_{i}^{1}$ or $I_{i}^{2}$.

Proposition 5.1. There exists $i \in\{1,2\}$ such that at the vertices of $G_{T}$ the edge class $\delta_{3-i}$ has endpoints in the external interval $I_{i}$. See Figure 5.1. 

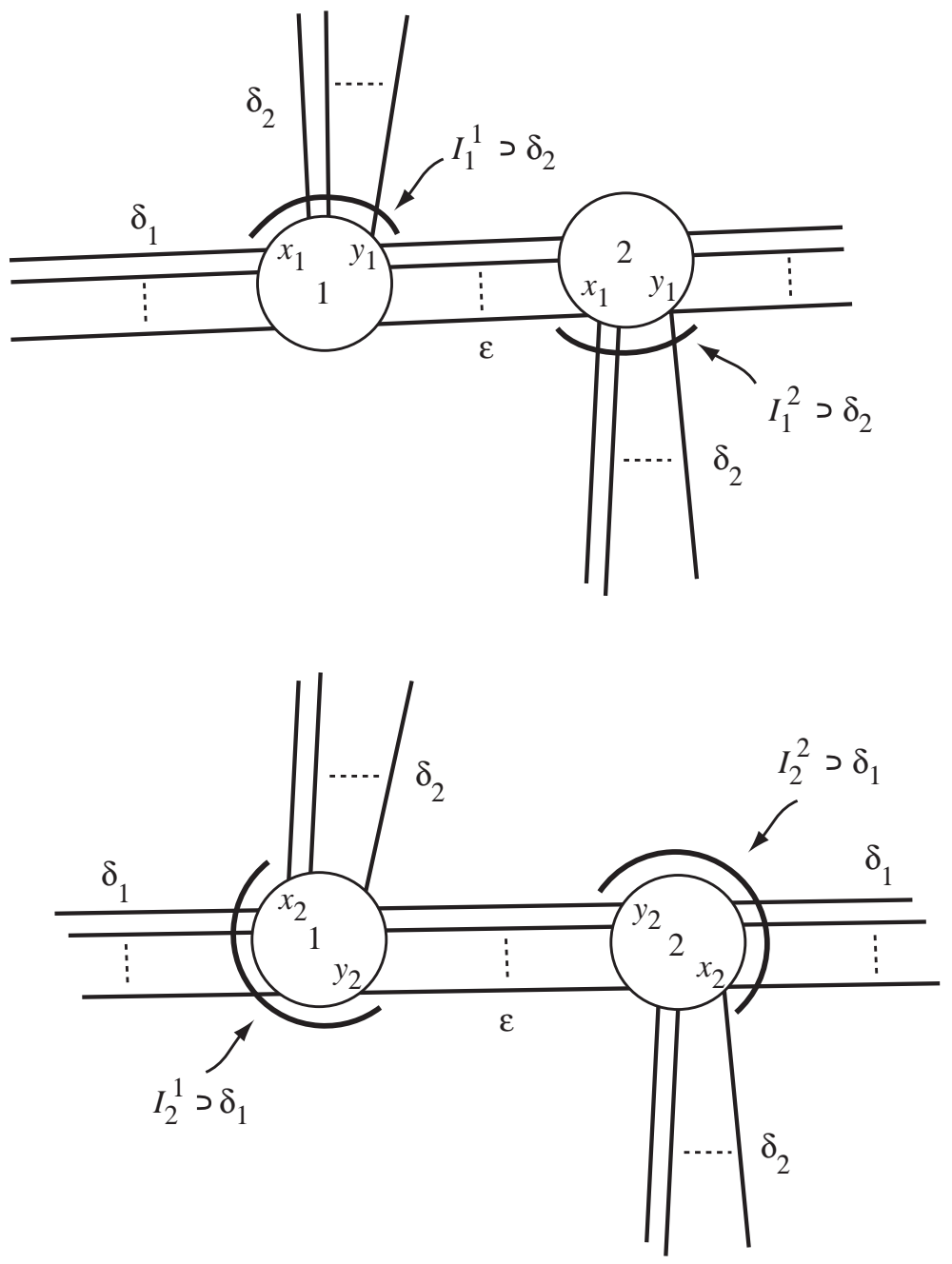

Figure 5.1.

If some $f_{i}$ is a bigon then the proposition is clear. So we assume not. Then $\left\{\varepsilon, \delta_{i}\right\}=\left\{\varphi_{i}, \psi_{i}\right\}$, where there are more edges of $f_{i}$ in class $\varphi_{i}$ than in class $\psi_{i}$ (see the Remark after Lemma 4.4). We assume also, for contradiction, that the edge class $\delta_{3-i}$ is not contained in the interval $I_{i}$ at the vertices of $G_{T}, i=1,2$.

Lemma 5.2. For any edge e of $G_{Q}$ in class $\delta_{3-i}$, the edges of $G_{Q}$ adjacent to $e$ on the $X_{i}$-side are in class $\varphi_{i}$. See Figure 5.2. 


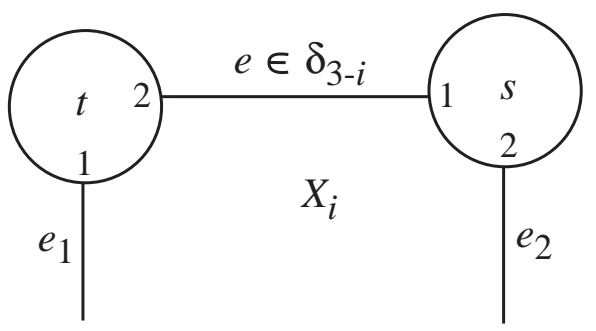

Figure 5.2.

Proof. We take $i=1$ and $X_{1}=B$ without loss of generality.

Let $p, q$ be the numbers of edges of $f_{1}$ in classes $\varphi_{1}, \psi_{1}$ respectively. Thus $p>q$. As in the proof of Lemma 4.2, consider the edges of $f_{1}$ as they lie on $G_{T}$, and number their endpoints at the vertices of $G_{T} 0,1, \ldots, n-1$, in anticlockwise (resp. clockwise) order around vertex 1 (resp. vertex 2). We may choose the numbering so that $I_{1}$ is $[0,1]$. See Figure 5.3.

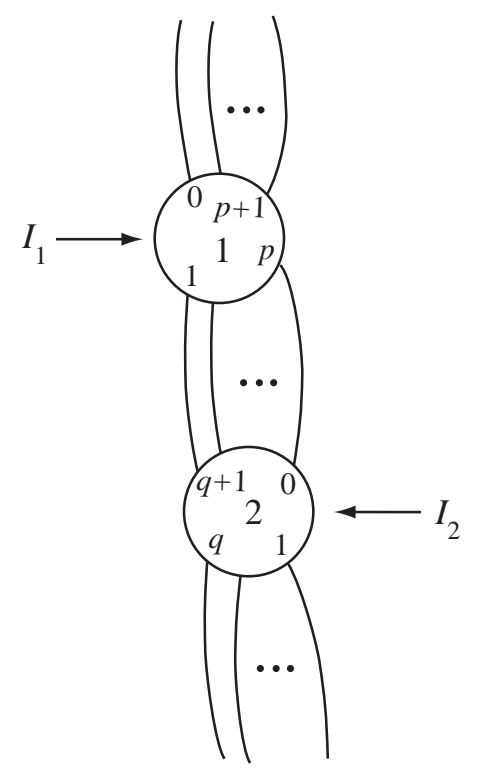

Figure 5.3.

Let $e$ be an edge of $G_{Q}$ in class $\delta_{2}$; as in Figure 5.2. Then $e$ appears in $G_{T}$ as in Figure 5.4.

Then by hypothesis, the label $s$ appears in the interval $(p, p+1)$ at vertex 1 . Since $p>q$, the edges of $f_{1}$ with endpoints $p, p+1$ at vertex 2 lie in the 


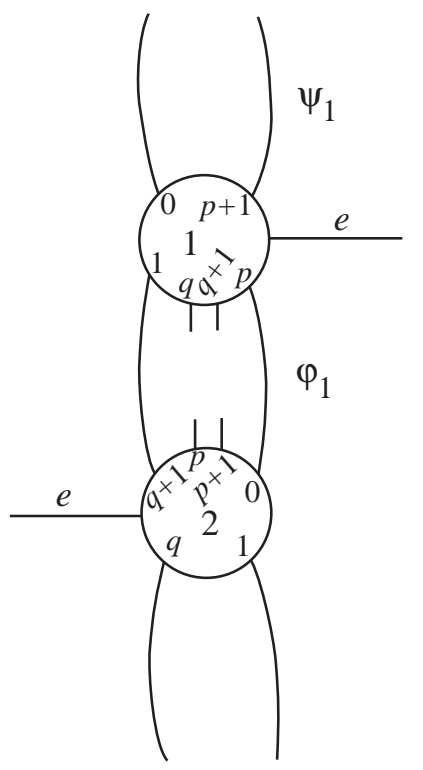

Figure 5.4.

class $\varphi_{1}$; see Figure 5.4. Since the endpoint of the edge $e_{2}$ at vertex 2 lies between $p$ and $p+1, e_{2}$ is in the class $\varphi_{1}$. Similarly, the label $t$ appears in $(q, q+1)$ at vertex 2 , and a completely analogous argument shows that the edge $e_{1}$ is also in the class $\varphi_{1}$.

Lemma 5.3. $\varphi_{i}=\delta_{i}, i=1,2$ (i.e., $f_{i}$ is good w.r.t. $\varepsilon$ ).

Proof. We show that $\varphi_{1}=\delta_{1}$; the proof that $\varphi_{2}=\delta_{2}$ is completely analogous.

Assume that $\varphi_{1}=\varepsilon$. Let $x$ be a vertex of $G_{Q}$ at which a $\left(\delta_{2}, \varepsilon\right)$ corner of the face $f_{2}$ is incident. Then, by Lemma 5.2 , we would have two edges in class $\varepsilon$ incident to $x$ at the same label, contradicting Lemma 3.3. See Figure 5.5.

Proof of Proposition 5.1. Let $x$ be a vertex of $G_{Q}$ at which an edge $e$ in class $\delta_{1}$ is incident. Then by two applications of Lemmas 5.2 and 5.3, we see that the edge incident to $x$ at the same label as $e$ is also in class $\delta_{1}$, contradicting Lemma 3.3. See Figure 5.6. 


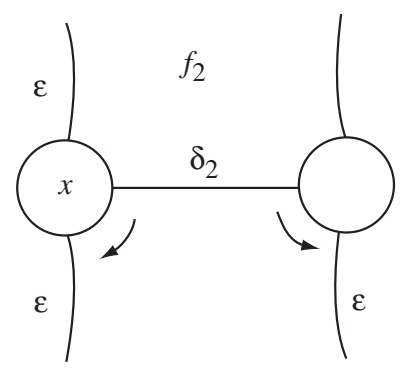

Figure 5.5.

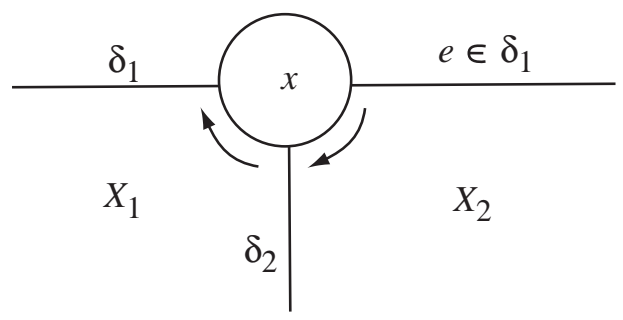

Figure 5.6.

Up to homeomorphism and relabelling, Proposition 5.1 allows us to assume that the edges of $f_{1}, f_{2}$ on $G_{T}$ are as in Figure 5.7 with the endpoints of edge class $\delta_{2}$ contained in $I_{1}$. Let $B_{\varepsilon}, B_{1}, B_{2}$ be bands of $G_{T}$ containing the edges in classes $\varepsilon, \delta_{1}, \delta_{2}$ respectively. Let $\left\{v_{1}, v_{2}\right\}$ be the vertices of $G_{T}$. Let $A_{i}$ be an annular neighborhood of $v_{1} \cup v_{2} \cup B_{\varepsilon} \cup B_{i}$ in $\widehat{T}$, such that each component of $\partial A_{1}$ meets each component of $\partial A_{2}$ in a single point; see Figure 5.8.

Let $A_{i}^{\prime}=\overline{\widehat{T}-A_{i}}$. Recall that $M=M_{1} \cup_{\widehat{T}} M_{2}$, and let $H_{i}=V \cap M_{i}$. Let $W_{i}=\operatorname{nhd}_{M_{i}}\left(A_{i} \cup H_{i} \cup f_{i}\right)$. Thus $\partial W_{i}=A_{i} \cup C_{i}$, where $C_{i}$ is an annulus with $\partial C_{i}=\partial A_{i}$. Note that $H_{i} \cap \partial W_{i}=v_{1} \cup v_{2}$. By Lemma 4.2, $W_{i}$ is a solid torus. Since $E(K)$ is atoroidal, $\overline{M_{i}-W_{i}}=W_{i}^{\prime}$ is also a solid torus.

Recall that the boundary components of the surface $Q \subset E(K)$ have slope $\mu$, the meridian of $K$. Let $h_{i}:\left(D^{2} \times I ; D^{2} \times\{0\}, D^{2} \times\{1\}\right) \rightarrow\left(H_{i} ; v_{1}, v_{2}\right)$ be a homeomorphism such that each (arc) component of $\partial Q \cap H_{i}$ is of the form $h_{i}(x \times I)$ for some $x \in \partial D^{2}$. For any $y \in \partial D^{2}$, we shall say that the arc $h_{i}(y \times I) \subset \partial H_{i}$ determines the $\mu$-framing on $H_{i}$. See Figure 5.9.

Recall that $I_{i}^{1}$ is the external interval of $f_{i}$ around $\partial v_{1}$. Pick a point $x_{i}^{1} \in \operatorname{Int} I_{i}^{1}$. Then $I_{i}^{2}=h_{i}\left(h_{i}^{-1}\left(I_{i}^{1}\right) \times\{1\}\right)$ is the corresponding external interval around $\partial v_{2}$, with $x_{i}^{2}=h_{i}\left(h_{i}^{-1}\left(x_{i}^{1}\right) \times\{1\}\right) \in \operatorname{Int} I_{i}^{2}$. Let $\xi_{i} \subset \partial H_{i}$ be 


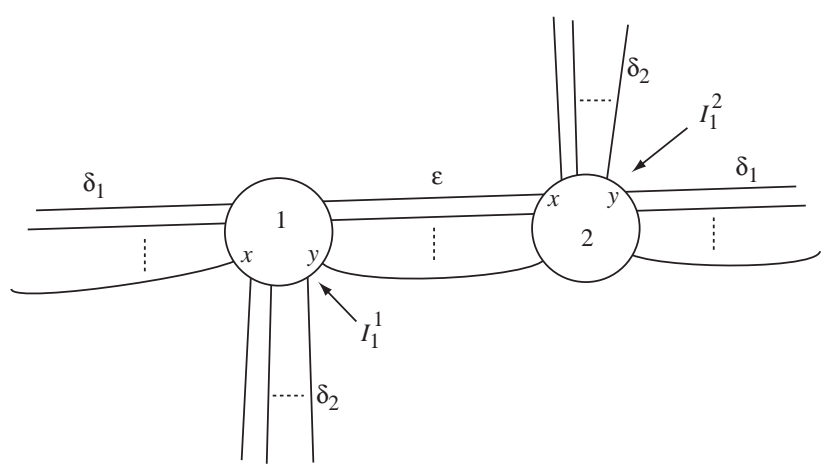

Figure 5.7.

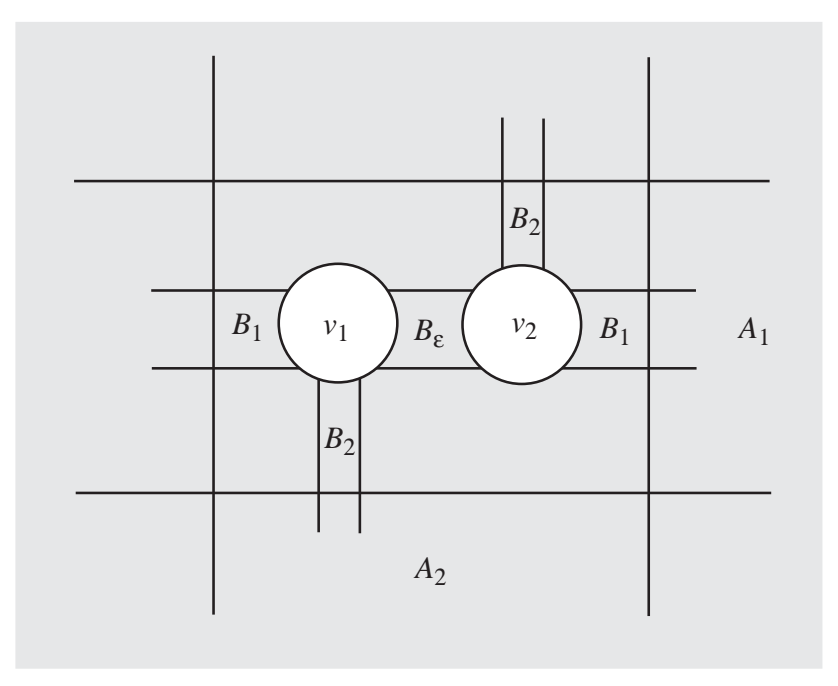

Figure 5.8.

the $\operatorname{arc} h_{i}\left(h_{i}^{-1}\left(x_{i}^{1}\right) \times I\right)$; thus $\partial \xi_{i}=\left\{x_{i}^{1}, x_{i}^{2}\right\}$ and $\xi_{i}$ determines the $\mu$-framing on $H_{i}$. Note that $\xi_{i} \cap \partial f_{i}=\emptyset$. Let $\gamma_{i}^{1}$ and $\gamma_{i}^{2}$ be arcs in $\overline{A_{1} \cap A_{2}-\left(v_{1} \cup v_{2}\right)}$ running from $x_{i}^{1}$ and $x_{i}^{2}$ respectively to $\partial A_{i}$, and disjoint from $\partial f_{i}$. Note that $\gamma_{i}^{1}$ and $\gamma_{i}^{2}$ go to different components of $\partial A_{i}$.

Let $B_{i}^{j}=\left(B_{i} \cap \partial v_{j}\right), j=1,2$. We are assuming $B_{2}^{j} \subset I_{1}^{j}, j=1,2$, (Proposition 5.1) so $\gamma_{1}^{1}$ and $\gamma_{1}^{2}$ are as shown in Figure 5.10. For $\gamma_{2}^{j}$ there are two cases:

Case I. $B_{1}^{j} \cap I_{2}^{j}=\emptyset, j=1,2$.

Case II. $B_{1}^{j} \subset I_{2}^{j}, j=1,2$. 


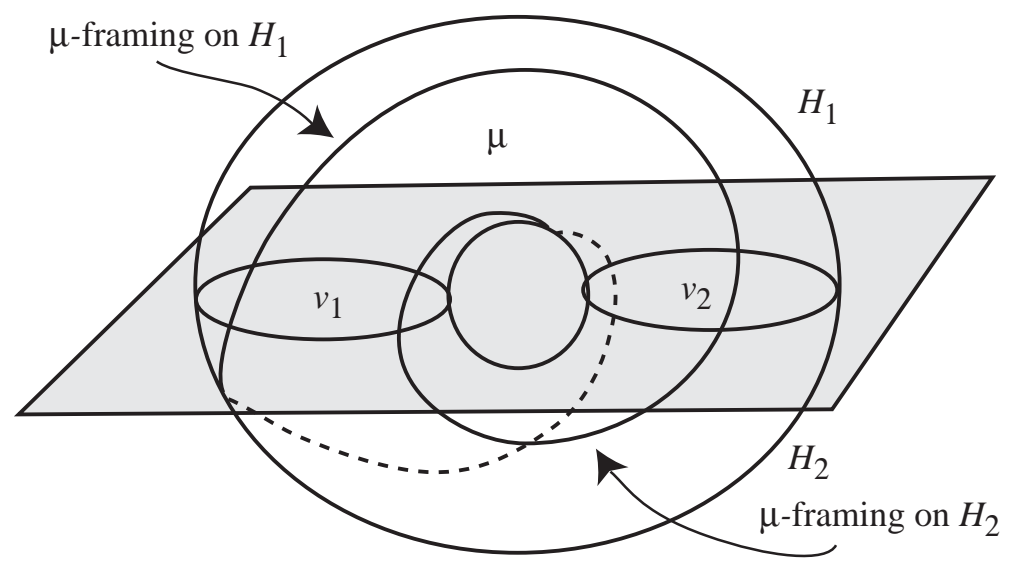

Figure 5.9.

In these cases, $\gamma_{2}^{1}$ and $\gamma_{2}^{2}$ are illustrated in Figures 5.11 and 5.12 respectively.

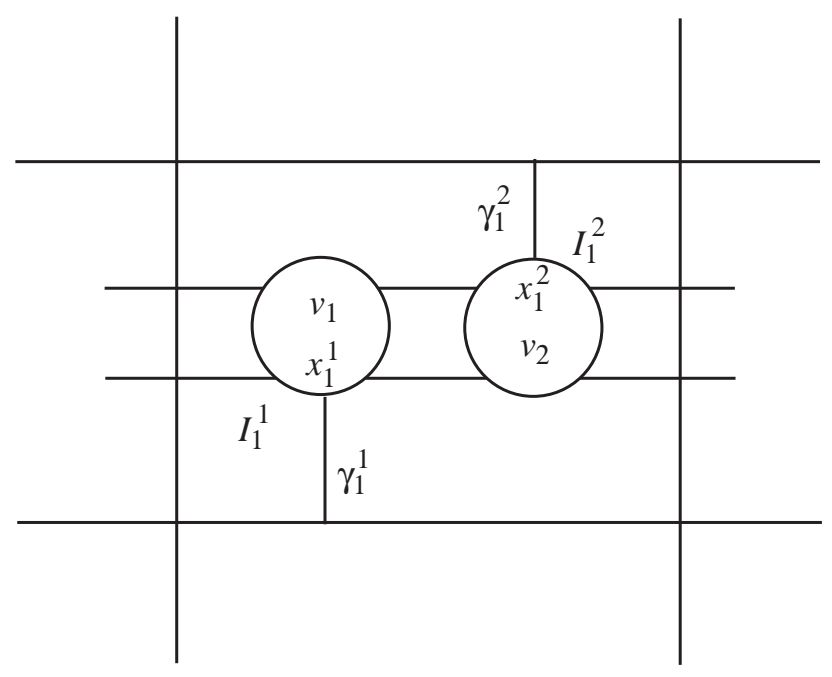

Figure 5.10.

Since $\left(\xi_{i} \cup \gamma_{i}^{1} \cup \gamma_{i}^{2}\right) \cap \partial f_{i}=\emptyset$, by definition of $W_{i}$ there is a disk (rectangle) $R_{i} \cong \xi_{i} \times I$ properly embedded in $\overline{W_{i}-H_{i}}$, with $\xi_{i} \times\{0\}=\xi_{i}, x_{i}^{j} \times I=\gamma_{i}^{j}$, $j=1,2$, and $\xi_{i} \times\{1\} \subset C_{i}$. See Figure 5.13 for the case $i=1$; the case $i=2$ is similar. 


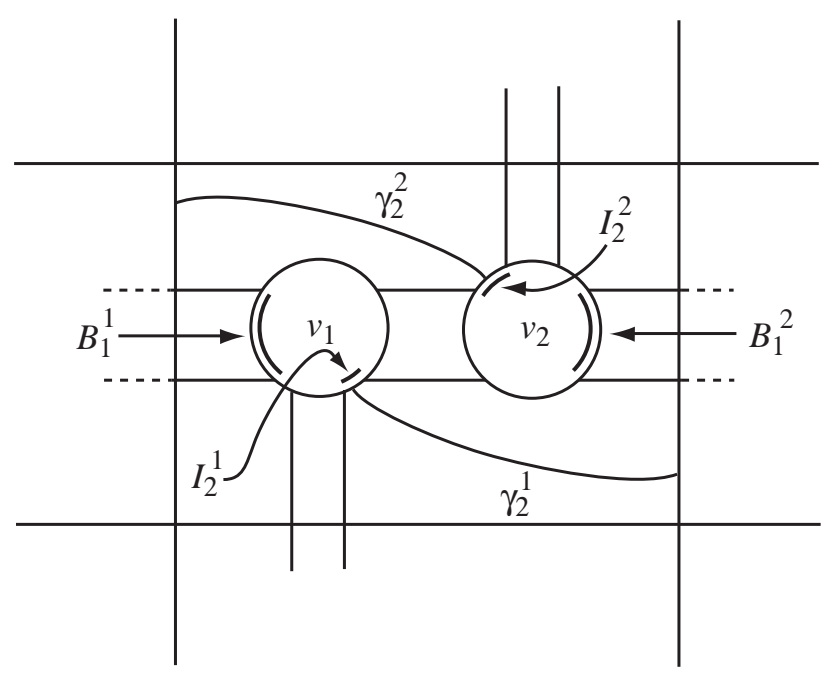

Figure 5.11.

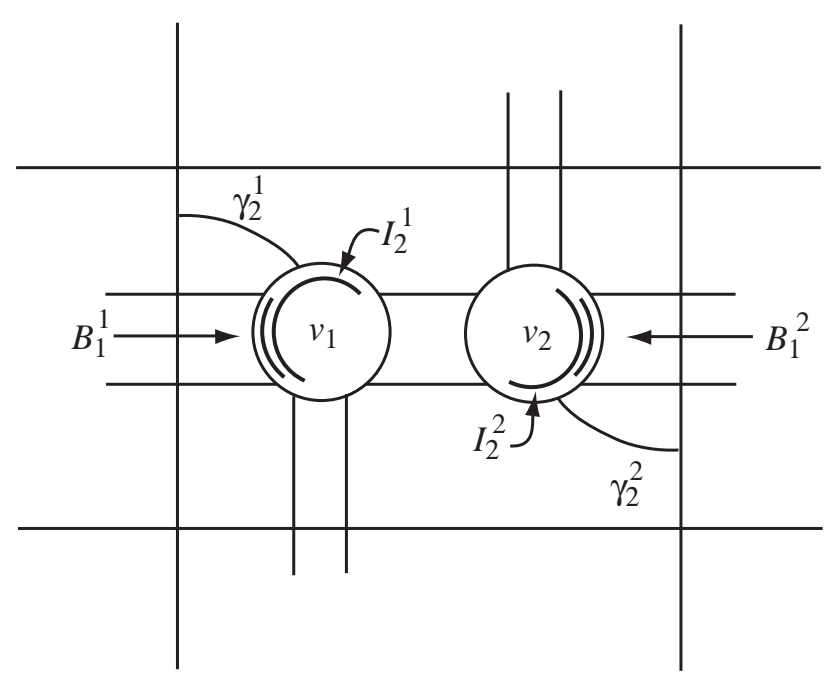

Figure 5.12.

We regard $\widehat{T} \cup C_{1} \cup C_{2} \cup R_{1} \cup R_{2} \cup V$ as a subset of $S^{3}$, in the following way. First, identify $\widehat{T}$ with a standardly embedded torus in $S^{3}$, separating $S^{3}$ into two solid tori $U_{1}$ and $U_{2}$, where the components of $\partial A_{1}$ are longitudes of $U_{1}$ and meridians of $U_{2}$, and vice versa for the components of $\partial A_{2}$. Under this identification, we take $v_{1}, v_{2}, B_{1}, B_{2}$ and $B_{\varepsilon}$ to the standard configuration shown in Figure 5.8. Then we identify $C_{i}$ with the obvious annulus in $U_{i}$, 


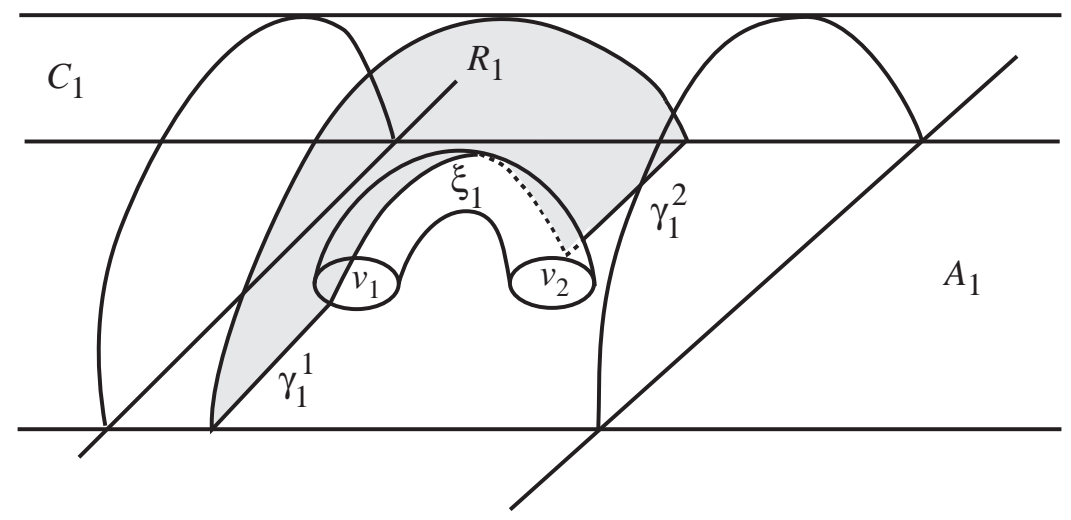

Figure 5.13.

separating $U_{i}$ into two solid tori $V_{i}$ and $V_{i}^{\prime}$, where $\partial V_{i}=A_{i} \cup C_{i}$, and $\partial V_{i}^{\prime}=$ $A_{i}^{\prime} \cup C_{i}$. See Figure 5.14.

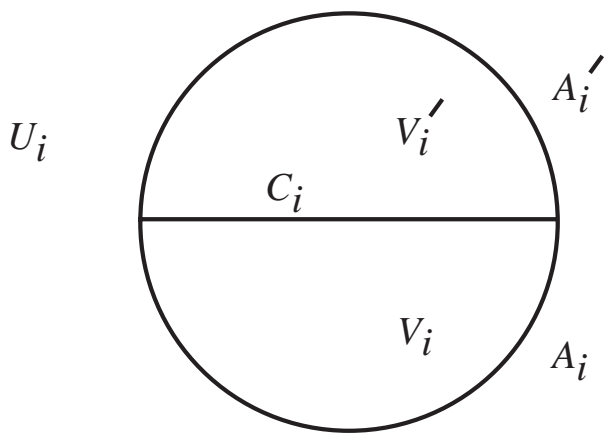

Figure 5.14.

Let $H_{i} \subset V_{i}$ be as shown in Figure 5.15.

Also embed $R_{i} \subset V_{i}$, as in Figure 5.13.

Let $K_{i}^{\prime}$ be a core of $V_{i}^{\prime}$, and let $K_{i}$ be a core of $V_{i}$ disjoint from $H_{i} \cup R_{i}$. See Figures 5.16 and 5.17 .

Note that $N\left(\widehat{T} \cup C_{1} \cup C_{2}\right)$ is the exterior of the link $L_{0}=K_{1} \cup K_{1}^{\prime} \cup K_{2} \cup K_{2}^{\prime}$; see Figure 5.18.

Since $W_{i}^{\prime}$ is a solid torus, we can regard $W_{i}^{\prime}$ as being obtained from $V_{i}^{\prime}$ by some Dehn surgery on $K_{i}^{\prime}$. Similarly, since $\overline{W_{i}-\operatorname{nhd}\left(R_{i} \cup H_{i}\right)}$ is a solid torus, we can regard $\left(W_{i} ; H_{i}, R_{i}\right)$ as being obtained from $\left(V_{i} ; H_{i}, R_{i}\right)$ by some Dehn surgery on $K_{i}$. Finally, let $K_{0}$ be the core of $V=H_{1} \cup H_{2} \subset S^{3}$. Then we have $\left(M, K_{\tau}\right)$ is obtained from $\left(S^{3}, K_{0}\right)$ by some Dehn surgery on $L_{0}$. 


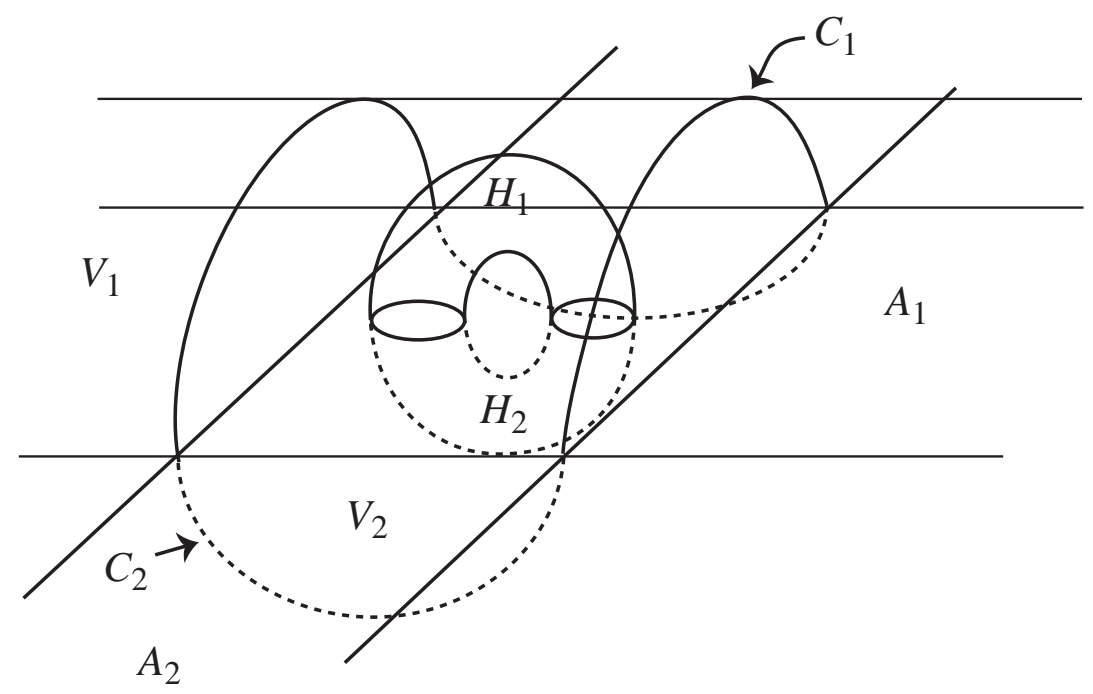

Figure 5.15.

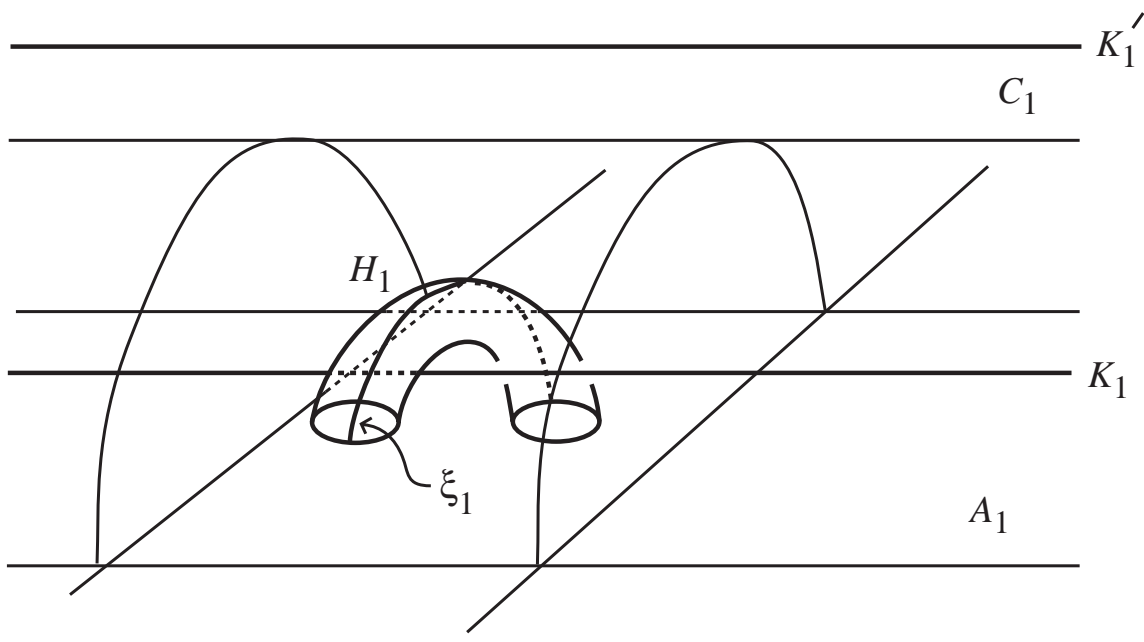

Figure 5.16.

That is, the exterior of $K$ is obtained from the exterior of $K_{0}$ in $S^{3}$ by a Dehn surgery on $L_{0}$.

Take the usual meridian-longitude basis for slopes on $\partial V$; i.e., the 0 framing of $K_{0}$ in $S^{3}$. Note that the meridian of $K_{0}$ corresponds to the slope $\tau$ on $\partial V$. Recall that the slope $\mu$ on $\partial V$ is the meridian of $K$. 


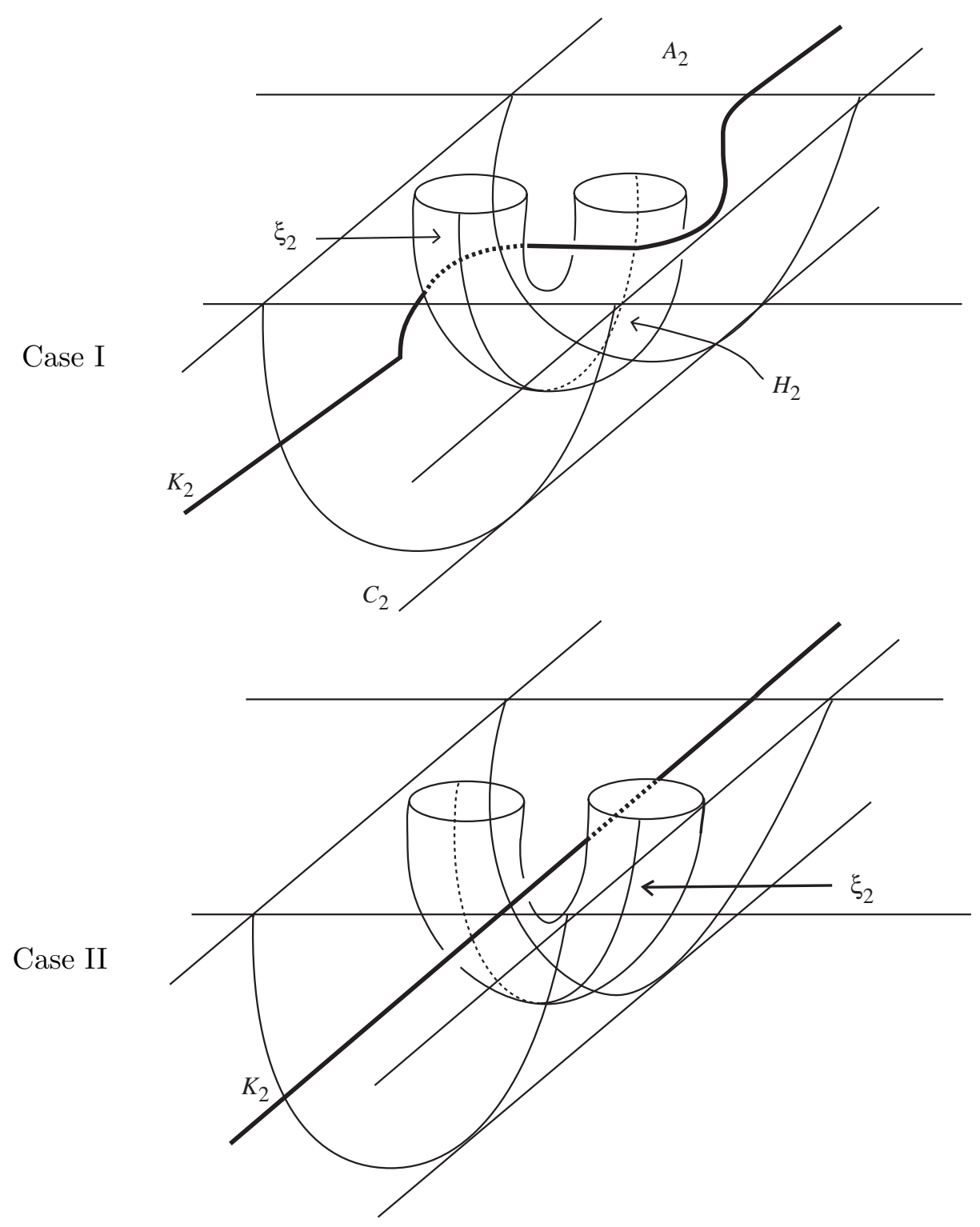

Figure 5.17.

Lemma 5.4. The exterior of $K$ is obtained from the exterior of $K_{0}$ in $S^{3}$ by Dehn surgery on $L_{0}$. Let $\mu$ be the meridian of $K$. With respect to the framing of $K_{0}$ described above, $\mu=1 / 2$ in Case $I$ and $-1 / 2$ in Case II. 


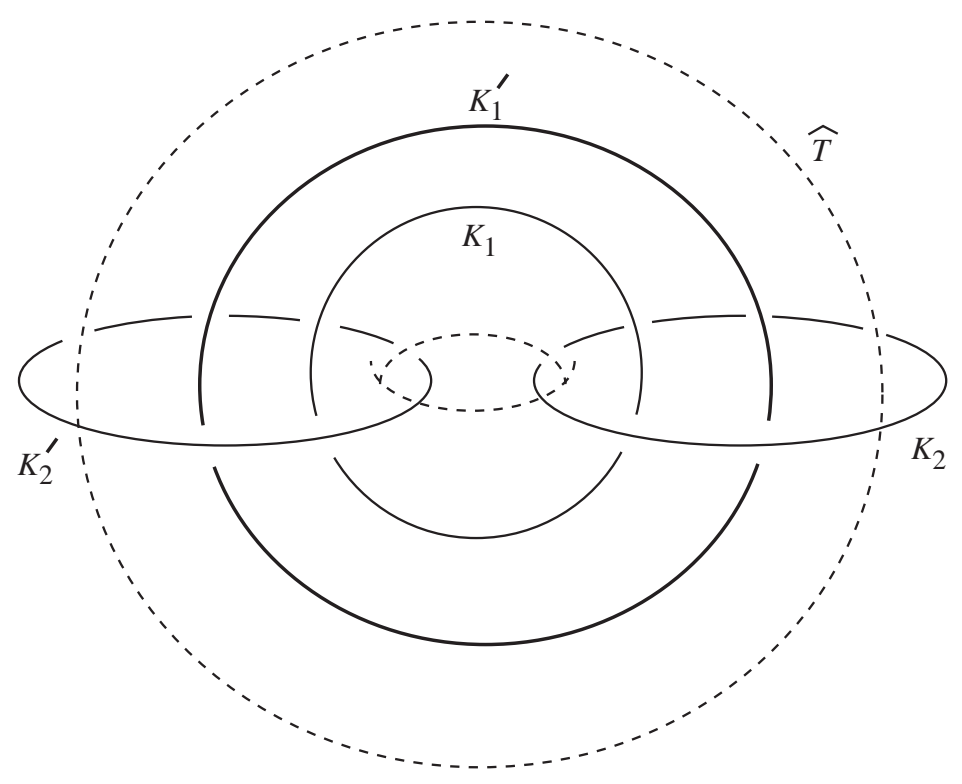

Figure 5.18.

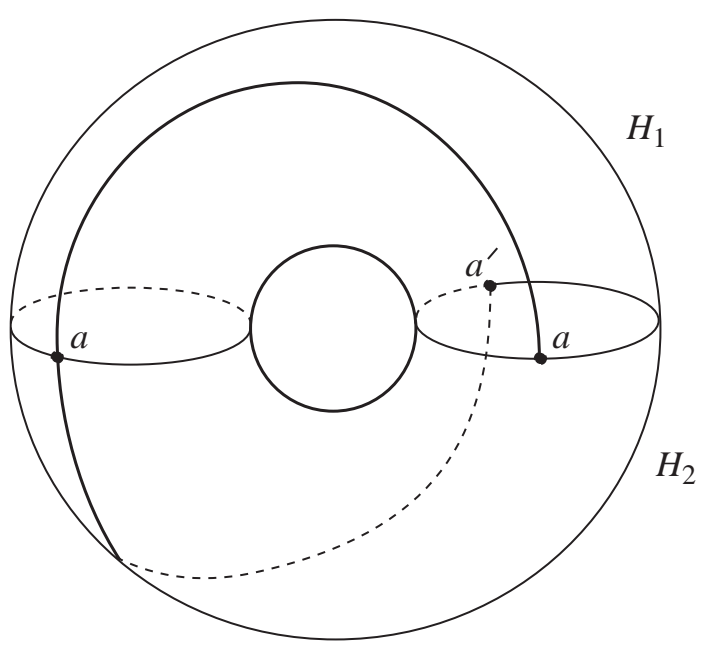

Figure 5.19.

First we adopt the following convention regarding labels around $\partial v_{1}$ and $\partial v_{2}$. Recall that each label $1,2, \ldots, q$ occurs twice around each of $\partial v_{1}$ and $\partial v_{2}$. Calling the two occurrences of some label $a$ and $a^{\prime}\left(a^{\prime \prime}=a\right)$, if $a$ is an occurrence of a label on $\partial v_{1}$ then we will denote by $a$ the point on 
$\partial v_{2}$ corresponding to $a$ with respect to the $\mu$-framing on $H_{1}$, and by $a^{\prime}$ the point on $\partial v_{2}$ corresponding to $a$ with respect to the $\mu$-framing on $H_{2}$. See Figure 5.19.

Let the labels of the endpoints of the external interval $I_{i}$ be $a_{i}$ and $b_{i}$, where $a_{i} \in B_{i}$ and $b_{i} \in B_{\varepsilon}$ at vertex $v_{1}$. For the next lemma, recall that labels are read anticlockwise around $v_{1}$ and clockwise around $v_{2}$.

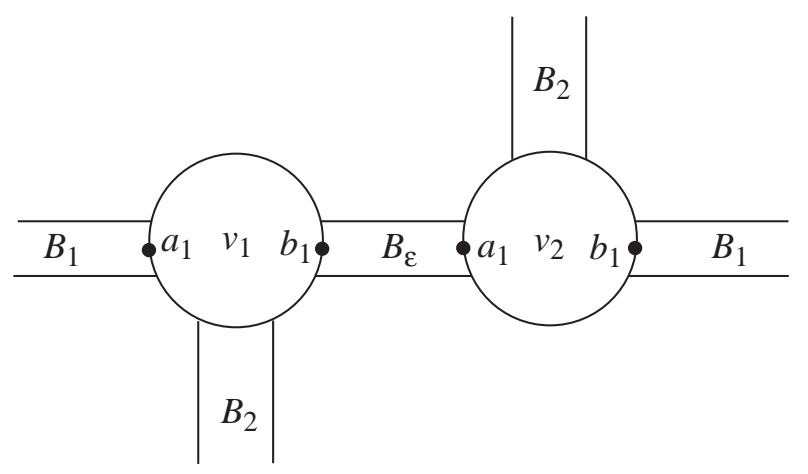

Figure 5.20.

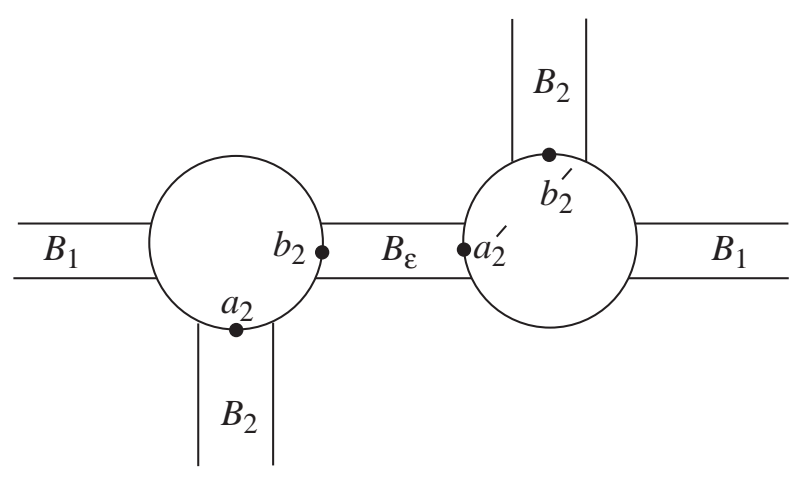

Figure 5.21.

Lemma 5.5. The labels $a_{1}, b_{2}, b_{2}^{\prime}$ occur in the order $a_{1} b_{2}^{\prime} b_{2}$.

Proof. By looking at vertex $v_{2}$, we see that we have order $a_{1} b_{2}^{\prime} b_{1}$. See Figures 5.20 and 5.21. Therefore, if we had order $a_{1} b_{2} b_{2}^{\prime}$, then we would have order $a_{1} b_{2} b_{2}^{\prime} b_{1}$. But at vertex $v_{1}, b_{1}$ and $b_{2}$ are in $B_{\varepsilon}$. Hence we would have 
$b_{2}^{\prime}$ in $B_{\varepsilon}$ at vertex $v_{1}$, giving $q+1$ parallel edges in $B_{\varepsilon}$. But this gives the contradiction that $K$ is cabled, using [11].
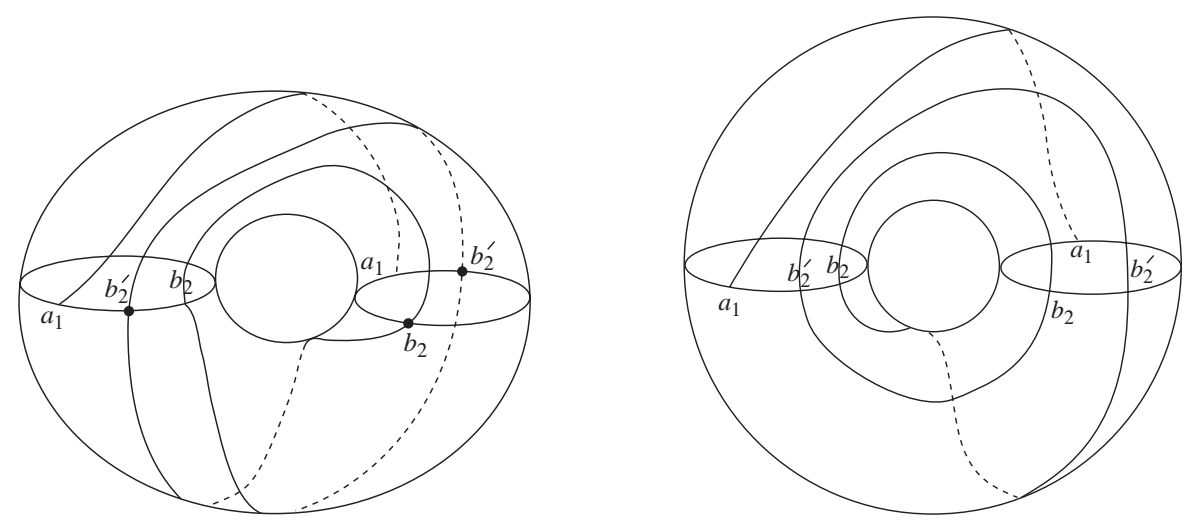

Figure 5.22.

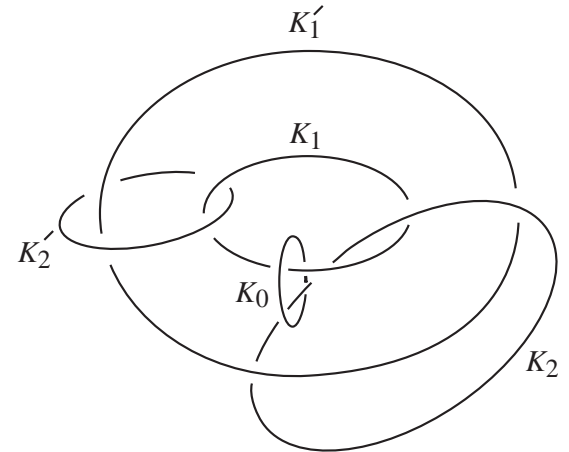

Case I

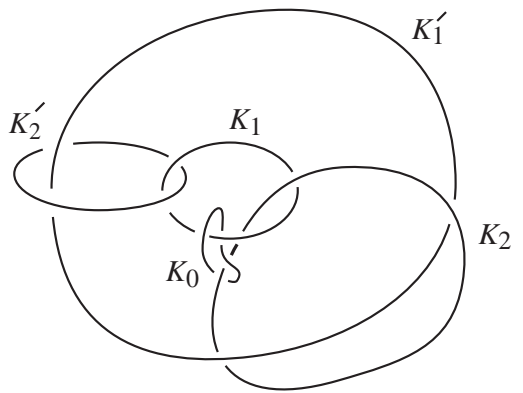

Case II

Figure 5.23.

Proof of Lemma 5.4. Consider the arc in $\partial H_{1}$ given by the $\mu$-framing on $H_{1}$ (see the arc $\xi_{1}$ in Figure 5.16) with endpoint $a_{1}$ at $v_{1}$, and the arc in $\partial H_{2}$ given by the $\mu$-framing on $\mathrm{H}_{2}$ (see the arc $\xi_{2}$ in Figure 5.17) with endpoint $b_{2}$ at $v_{1}$. See Figure 5.22. Using Lemma 5.5, we see that the meridian $\mu$ corresponding to the label $b_{2}$ is $1 / 2$ in Case I and $-1 / 2$ in Case II. See Figure 5.22.

(Lemma 5.4)

Let $L$ be the link $K_{1} \cup K_{1}^{\prime} \cup K_{2} \cup K_{2}^{\prime} \cup K_{0}$; see Figure 5.23. 
One can check that $L$ in Case II is the reflection of $L$ in Case I (in which $K_{i} \mapsto K_{i}, 0 \leq i \leq 2$, and $K_{i}^{\prime} \mapsto K_{i}^{\prime}, i=1,2$.). Note that under this reflection the (1/2)-framing on $K_{0}$ goes to the $(-1 / 2)$-framing (and the longitude to the longitude). As we are interested in identifying the exterior of $K$, we will assume from now on without loss of generality that we are in Case I.

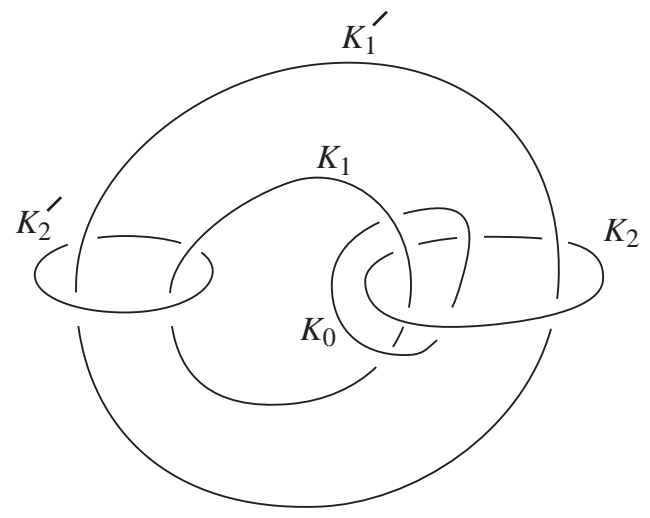

Figure 5.24: The link $L$

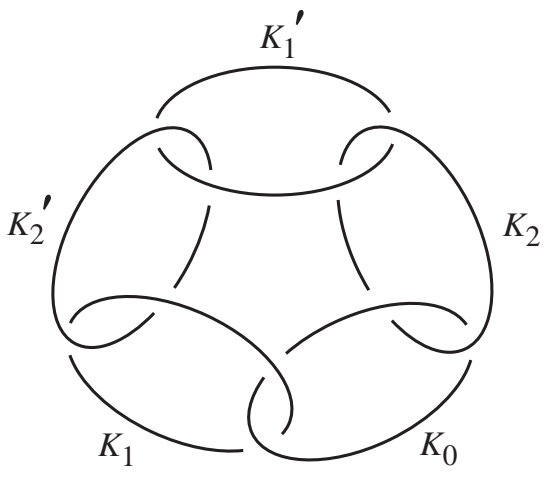

Figure 5.25: Minimally twisted 5-chain

Let $L(\alpha, \beta, \gamma, \delta, \varepsilon)$ be the Dehn filling on the exterior of $L$ where $\alpha, \beta, \gamma, \delta, \varepsilon$ denote the filling slopes on $K_{1}, K_{1}^{\prime}, K_{2}, K_{2}^{\prime}, K_{0}$ respectively. When one of the filling slopes is an asterisk $(*)$, then no filling is done on the corresponding component.

Proposition 5.6. Let $K$ be a hyperbolic knot in the 3 -sphere that admits a non-integral surgery containing an incompressible torus. Then $E(K)=$ 
$L(\alpha, \beta, \gamma, \delta, *)$ where $L$ is as in Figure 5.24. Furthermore $S^{3}=L\left(\alpha, \beta, \gamma, \delta, \frac{1}{2}\right)$, and the non-integral surgery is $L\left(\alpha, \beta, \gamma, \delta, \frac{1}{0}\right)$. Finally, $\Delta\left(\chi, \frac{0}{1}\right) \geq 2$ for $\chi \in\{\alpha, \beta, \gamma, \delta\}$. Conversely, any $\alpha, \beta, \gamma, \delta$ such that $L\left(\alpha, \beta, \gamma, \delta, \frac{1}{2}\right)=S^{3}$ and such that $\Delta\left(\chi, \frac{0}{1}\right) \geq 2$ for each $\chi \in\{\alpha, \beta, \gamma, \delta\}$ gives rise to a knot $K$ in $S^{3}$ admitting a non-integral toroidal surgery.

Proof. Given the above discussion, to prove the first implication we need only show that $\Delta\left(\chi, \frac{0}{1}\right) \geq 2$. This follows immediately from the observation that $L\left(\alpha, \beta, \gamma, \delta, \frac{1}{0}\right)=M_{1} \cup_{\widehat{T}} M_{2}$ where $M_{1}\left(M_{2}\right)$ is obtained from $D^{2} \times S^{1}$ by Dehn surgery along two circle fibers $K_{1}, K_{1}^{\prime}\left(K_{2}, K_{2}^{\prime}\right)$. The circle fibration of the exterior of $K_{1} \cup K_{1}^{\prime}\left(K_{2} \cup K_{2}^{\prime}\right)$ gives rise to a longitudinal framing of $K_{1}, K_{1}^{\prime}$ $\left(K_{2}, K_{2}^{\prime}\right)$. Thus $\widehat{T}$ will be incompressible in $M_{1}\left(M_{2}\right)$ only if $\Delta\left(\alpha, \frac{0}{1}\right) \geq 2$ and $\Delta\left(\beta, \frac{0}{1}\right) \geq 2\left(\Delta\left(\gamma, \frac{0}{1}\right) \geq 2\right.$ and $\Delta\left(\delta, \frac{0}{1}\right) \geq 2$, respectively). The converse follows similarly.

Remark. By twisting once along the disk bounded by $K_{0}$ one sees that $L$ has the same exterior as the minimally twisted 5-chain, $L^{\prime}$, pictured in Figure 5.25. Thus Proposition 5.6 says that $K$ can be written as a Dehn surgery on four components of the minimally twisted 5 -chain. In this picture the $S^{3}$ surgery is $1 / 1$ on $K_{0}$ and the toroidal surgery is $-1 / 1$.

\section{The tangle covered by $K$.}

Proposition 5.6 says that $K$ is obtained by Dehn surgery on $L$. Both $K$ and $L$ are strongly invertible and the quotients of their exteriors under these involutions give rise to a pair of tangles. The fact that the exterior of $K$ is obtained by Dehn filling the exterior of $L$, means that the tangle covered by $K$ is a tangle filling of the tangle covered by $L$. By analyzing tangle fillings we prove (Theorem 1.1) that the tangle covered by $K$ must be one of the tangles $B(\ell, m, n, p)$ of [6], and, consequently that $K$ is one of the knots $k(\ell, m, n, p)$ described by Eudave-Muñoz in [6]. We begin with a general discussion of tangles.

In our conventions on rational tangles we follow [6] and [7]. There is a 1-1 correspondence between rational tangles and $\mathbb{Q} \cup\{\infty\}$. Denote by $R(p / q)$ the rational tangle determined by $\frac{p}{q} \in \mathbb{Q} \cup\{\infty\}$. See Figure 6.1. $p / q$ is called the slope of $R(p / q)$.

The distance between two tangle slopes, $\Delta\left(\frac{p_{1}}{q_{1}}, \frac{p_{2}}{q_{2}}\right)$, is defined to be $\mid p_{1} q_{2}-$ $p_{2} q_{1} \mid$. 

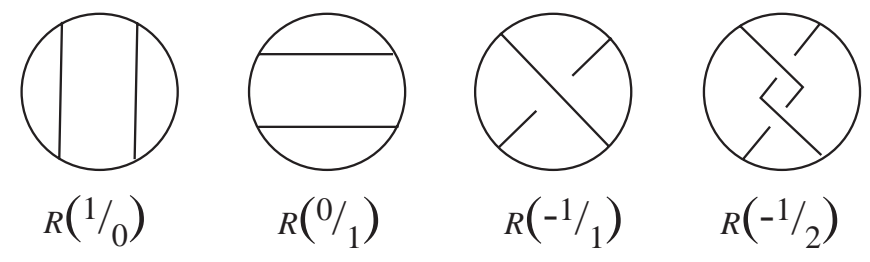

Figure 6.1.

Remark. A rational tangle can be represented as a pair of arcs on the boundary of the tangle ball. Let $t_{1}$ be one of these arcs from $R\left(p_{1} / q_{1}\right)$ and $t_{2}$ be one from $R\left(p_{2} / p_{2}\right)$ represented on the same tangle sphere. Isotop $t_{1}, t_{2}$ (rel endpoints) so that they intersect minimally. Let $e$ be the number of endpoints shared by $t_{1}, t_{2}$ and let $p$ be the number of interior points of intersection. Then $\Delta\left(\frac{p_{1}}{q_{1}}, \frac{p_{2}}{q_{2}}\right)=e+2 p$. To check the representation of a tangle as a rational number, up to sign, it is useful to remember that $|p|=\Delta\left(\frac{p}{q}, \frac{0}{1}\right)$, $|q|=\Delta\left(\frac{p}{q}, \frac{1}{0}\right)$.

It is convenient to generalize the usual notion of a tangle, as follows. A tangle $\mathcal{T}$ is a pair $(X, A)$, where $X$ is $S^{3}$ minus the interiors of $n$ disjoint 3 -balls, and $A$ is a properly embedded 1-manifold in $X$, meeting each component of $\partial X$ in exactly four points. The term rational tangle will retain its usual meaning. Given $\alpha_{i} \in \mathbb{Q} \cup\{1 / 0\}, 1 \leq i \leq n, \mathcal{T}\left(\alpha_{1}, \alpha_{2} \ldots, \alpha_{n}\right)$ will denote the link in $S^{3}$ obtained by inserting the rational tangle $R\left(\alpha_{i}\right)$ into the $i$ th puncture of $\mathcal{T}$. In this paper we consider the tangles of Figures 6.2 and 6.3. The tangle shown in Figure 6.2 will be referred to as $\mathcal{Q}$ hereafter. A filling of $\mathcal{Q}, \mathcal{Q}(\alpha, \beta, \gamma, \delta)$, refers to filling punctures $A, B, C, D$ with rational tangles of slope $\alpha, \beta, \gamma, \delta$ (resp.). The tangle of Figure 6.3 is called the pentangle and will be referred to as $\mathcal{P}$ and, similarly, a filling thereof as $\mathcal{P}(\alpha, \beta, \gamma, \delta, \varepsilon)$. Note that $\mathcal{Q}(\alpha, \beta, \gamma, \delta)=\mathcal{P}\left(\alpha, \beta, \gamma, \delta, \frac{2}{1}\right)$.

Often we will insert rational tangles into only a subset of the punctures of $\mathcal{T}$, giving a new tangle. We denote this, $\mathcal{T}\left(r_{1}, r_{2}, \ldots, *, \ldots\right)$, where an asterisk indicates that no tangle is inserted in that puncture. For example, the tangle $\mathcal{Q}(\alpha, *, \gamma, *)$ is that obtained from $\mathcal{Q}$ by replacing punctures $A, C$ with tangles $R(\alpha), R(\gamma)$ (resp.) and leaving punctures $B, D$ alone.

The tangle $\mathcal{Q}$ admits a symmetry by rotation in the horizontal axis, $r_{h}$, which interchanges $A, C$ and $B, D$. It also admits the symmetry by rotation in the vertical axis, $r_{v}$, which interchanges $A, B$ and $C, D$. We will refer to these symmetries by their action on $(A, B, C, D)$ :

$$
r_{h}=(C, D, A, B), \quad r_{v}=(B, A, D, C), \quad r_{h} r_{v}=(D, C, B, A)
$$




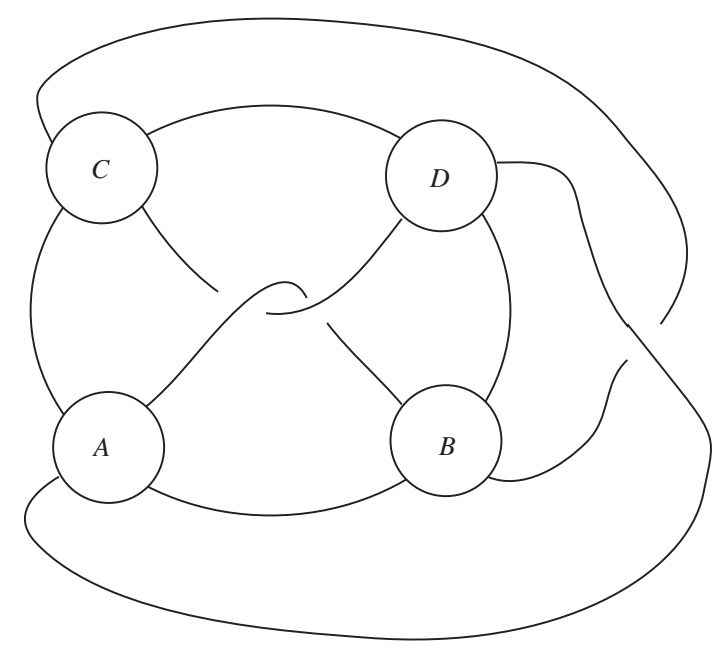

Figure 6.2: The tangle $\mathcal{Q}$

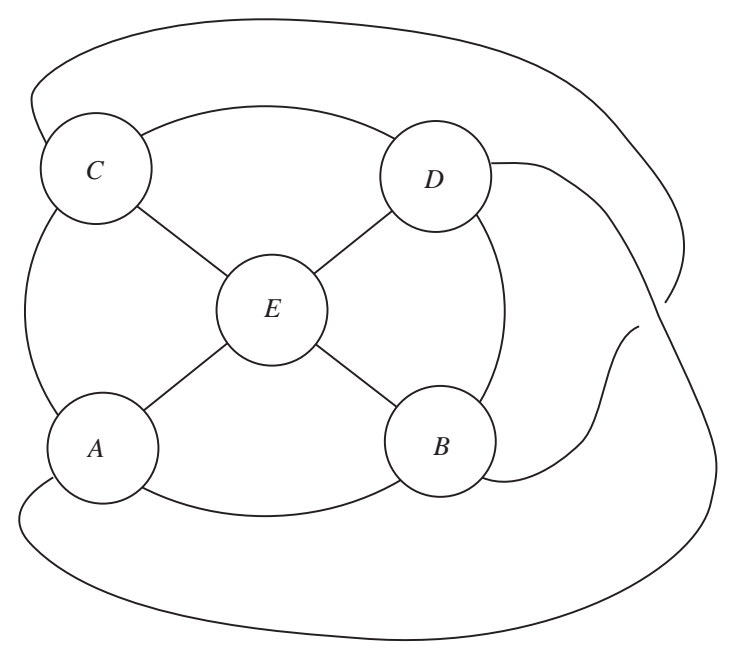

Figure 6.3: The pentangle $\mathcal{P}$

Note that these symmetries preserve the coordinates on the tangle balls $A, B, C, D$ (i.e., $\frac{0}{1} \rightarrow \frac{0}{1}, \frac{1}{0} \rightarrow \frac{1}{0}$ ). Also we can take any boundary component of $\mathcal{Q}$ to any other by one of these symmetries.

The next two sections will provide support for the proof of the following.

Proposition 6.1. Assume $\mathcal{Q}(\alpha, \beta, \gamma, \delta)$ is the unknot. If $\Delta\left(\chi, \frac{1}{0}\right) \geq 2$ for each $\chi \in\{\alpha, \beta, \gamma, \delta\}$, then $\frac{1}{2} \in\{\alpha, \beta, \gamma, \delta\}$. 
Proof of Proposition 6.1. We assume that $\alpha, \beta, \gamma, \delta$ are tangle slopes as in the hypothesis of Proposition 6.1. By Corollary 8.5, we assume that $\Delta\left(\chi, \frac{1}{0}\right) \geq 3$ for $\chi \in\{\alpha, \beta, \gamma, \delta\}$.

Note that if $r$ is a slope, then the conditions $\Delta\left(r, \frac{0}{1}\right) \leq 1, \Delta\left(r, \frac{1}{1}\right) \leq 1$, and $\Delta\left(r, \frac{1}{0}\right) \geq 3$ cannot all three hold. Thus applying Proposition 8.6 under the symmetries $(A, B, C, D),(B, A, D, C),(C, D, A, B)$, and $(D, C, B, A)$, one of the following must hold:

a) $\Delta\left(\chi, \frac{0}{1}\right)=1$ for $\chi \in\{\alpha, \beta, \gamma, \delta\}$;

b) $\Delta\left(\chi, \frac{1}{1}\right)=1$ for $\chi \in\{\alpha, \beta, \gamma, \delta\}$;

c) $\Delta\left(\chi, \frac{0}{1}\right)=1$ for $\chi \in\{\alpha, \beta\}$ and $\Delta\left(\chi, \frac{1}{1}\right)=1$ for $\chi \in\{\gamma, \delta\}$;

d) $\Delta\left(\chi, \frac{0}{1}\right)=1$ for $\chi \in\{\gamma, \delta\}$ and $\Delta\left(\chi, \frac{1}{1}\right)=1$ for $\chi \in\{\alpha, \beta\}$.

Note that $\chi \neq \frac{0}{1}, \frac{1}{1}$ for $\chi \in\{\alpha, \beta, \gamma, \delta\}$ since $\Delta\left(\chi, \frac{1}{0}\right) \geq 3$.

Theorems 8.7 and 8.8 of Section 8 prove Proposition 6.1 in cases (a) and (b). Theorem 8.9 and the corresponding statement under the symmetry $(C, D, A, B)$, prove the Proposition in cases (c), (d).

The symmetries $r_{v}, r_{h}$, and $r_{v} r_{h}$ described above are also symmetries of $\mathcal{P}$ leaving $E$ invariant.

Proposition 6.2. Let $\alpha, \beta, \gamma, \delta$ be tangle slopes such that $\mathcal{P}\left(\alpha, \beta, \gamma, \delta, \frac{0}{1}\right)$ is the union of prime tangles and $\mathcal{P}\left(\alpha, \beta, \gamma, \delta, \frac{2}{1}\right)$ is the unknot. Then $\delta=1 / 2$, up to symmetry by $r_{h}, r_{v}$, or $r_{h} r_{v}$. Furthermore, $\mathcal{P}(\alpha, \beta, \gamma, \delta, *)$ is one of the tangles $B(\ell, m, n, p)$ of [6].

Proof. Recall that $\mathcal{P}\left(\alpha, \beta, \gamma, \delta, \frac{2}{1}\right)=\mathcal{Q}(\alpha, \beta, \gamma, \delta)$. The fact that $\mathcal{P}\left(\alpha, \beta, \gamma, \delta, \frac{0}{1}\right)$ is a union of prime tangles implies that $\Delta\left(\chi, \frac{1}{0}\right) \geq 2$ for each $\chi \in\{\alpha, \beta, \gamma, \delta\}$ (see for example the arguments of section 5 in [7]). Thus by Proposition 6.1 , up to symmetry, $\delta=1 / 2$. Now $\mathcal{P}\left(\alpha, \beta, \gamma, \frac{1}{2}, *\right)$ is the tangle $\mathcal{B}(A, B, C)$ discussed in section 5 of [7], up to change in tangle labels and tangle coordinates (see Figure 6.4). ${ }^{1}$ Furthermore under this correspondence $\mathcal{B}(A, B, C)+R\left(\frac{1}{2}\right)$ is $\mathcal{P}\left(\alpha, \beta, \gamma, \frac{1}{2}, \frac{0}{1}\right)$ and $\mathcal{B}(A, B, C)+R(\mu)$ is $\mathcal{P}\left(\alpha, \beta, \gamma, \frac{1}{2}, \frac{2}{1}\right)$. This is exactly the context of section 5 of [7] in which it is shown that such $\mathcal{B}(A, B, C)$ are exactly those tangles $B(\ell, m, n, p)$ of $[6]$.

\footnotetext{
${ }^{1}$ Figures 9 and $10(\mathrm{~b})$ of [7] should be reflected; thus $\mathcal{B}(A, B, C)$ is actually the mirror image of the tangle shown in Figure 9(a). We are grateful to Mario EudaveMuñoz for discussions on this point.
} 

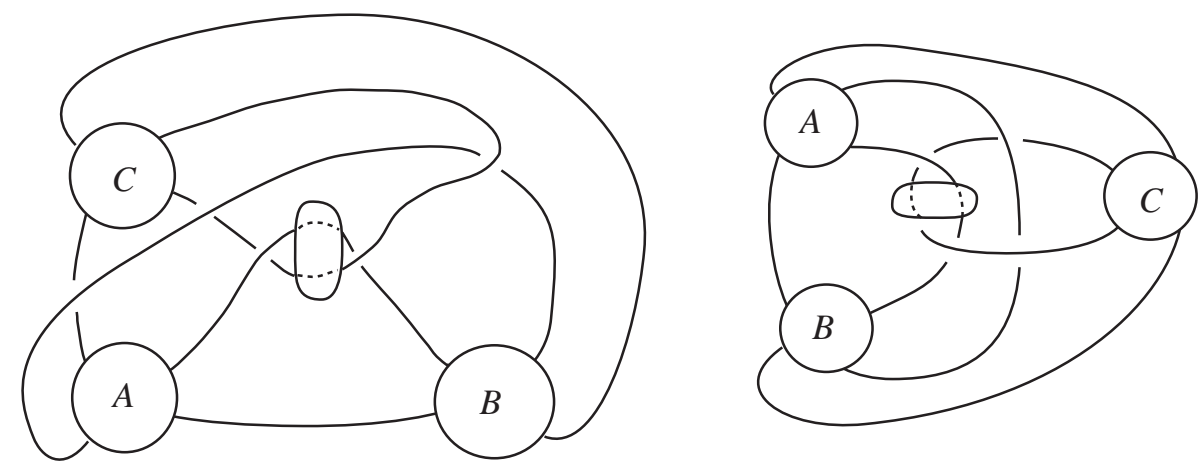

Figure 6.4: $\mathcal{P}\left(\alpha, \beta, \gamma, \frac{1}{2}, *\right)=\mathcal{B}(A, B, C)$

Let $\mathcal{T}\left(\alpha_{1}, \ldots, \alpha_{n}\right)$ be a filling of the tangle $\mathcal{T}$. The double branched cover of $S^{3}$ along the link $\mathcal{T}\left(\alpha_{1}, \ldots, \alpha_{n}\right)$ is a closed 3-manifold $\tilde{\mathcal{T}}\left(\alpha_{1}, \ldots, \alpha_{n}\right)$. In such a cover each tangle ball $R\left(\alpha_{i}\right)$ lifts to a solid torus, $\tilde{R}\left(\alpha_{i}\right)$. In the same way, the double branched cover $\tilde{\mathcal{T}}$ of $\mathcal{T}$ is a 3 -manifold with torus boundary components. That is, $\tilde{\mathcal{T}}$ is the exterior of a link in $\tilde{\mathcal{T}}\left(\alpha_{1}, \ldots, \alpha_{n}\right)$. In particular when $\mathcal{T}\left(\alpha_{1}, \ldots, \alpha_{n}\right)$ is the unknot, $\tilde{\mathcal{T}}\left(\alpha_{1}, \ldots, \alpha_{n}\right)$ is $S^{3}$, and $\tilde{\mathcal{T}}$ is the exterior of an $n$-component link in $S^{3}$. In any case, the double branched cover of any filling of $\mathcal{T}, \mathcal{T}\left(\alpha_{1}, \ldots, \alpha_{n}\right)$, is obtained from $\tilde{\mathcal{T}}$ by Dehn filling. In particular, if we lift the tangle coordinates from $\partial \mathcal{T}$ to $\partial \tilde{\mathcal{T}}, \tilde{\mathcal{T}}\left(\alpha_{1}, \ldots, \alpha_{n}\right)$ becomes the Dehn filling on $\tilde{\mathcal{T}}$ with filling co-ordinates $\alpha_{1}, \ldots, \alpha_{n}$, so there is no ambiguity in the notation $\tilde{\mathcal{T}}\left(\alpha_{1}, \ldots, \alpha_{n}\right)$. With this convention note that distance between tangle slopes on a boundary component of $\mathcal{T}$ is the same as the distance between the corresponding filling slopes on $\partial \tilde{\mathcal{T}}$.

By Proposition 6.2, the tangle $\mathcal{P}(\alpha, \beta, \gamma, \delta, *)$ is the same as the tangle $B(\ell, m, n, p)$ of [6]. The double branched cover of $B(\ell, m, n, p)$ is the exterior of the knot $k(\ell, m, n, p)$ of [6]. That is, $\tilde{\mathcal{P}}(\alpha, \beta, \gamma, \delta, *)$ is the exterior of some $k(\ell, m, n, p)$. Eudave-Muñoz shows that each $k(\ell, m, n, p)$ is a hyperbolic knot admitting a half-integral surgery with an incompressible torus. The following converse is the main result of the paper, which we restate here for the reader's convenience.

Theorem 1.1. Let $K$ be a hyperbolic knot in the 3-sphere that admits a non-integral surgery containing an incompressible torus. Then $K$ is one of the Eudave-Muñoz knots $k(\ell, m, n, p)$, and the surgery is the corresponding half-integral surgery.

Proof. Assume that $K$ is a knot in the 3-sphere admitting a non-integral 
surgery, $K(\tau)=M$, containing an incompressible torus. By Proposition 5.6, $E(K)=L(\alpha, \beta, \gamma, \delta, *)$. Figure 6.5 shows that $L$ is strongly invertible and depicts the quotient along with its fixed set. That quotient of $\left(S^{3}, L\right)$ under this strong inversion is $\mathcal{P}\left(\frac{0}{1}, \frac{1}{1}, \frac{0}{1}, \frac{1}{1}, \frac{0}{1}\right)$, where invariant regular neighborhoods of $L$ become tangle balls in the quotient. That is, the quotient of $E(L)$ under the involution is the pentangle $\mathcal{P}$. As indicated by the dashed line framings of the tangle balls in $\mathcal{P}$ of Figure 6.5, the longitude framings of $\partial E(L)$ pass to the $\frac{1}{0}$ framings of the tangle balls. As indicated by the solid lines (of the unknot) the meridians of $\partial E(L)$ pass to either the $\frac{0}{1}$ or $\frac{1}{1}$ framings of the quotient tangle. In particular, the (meridian, longitude)-framing of $K_{0}$ passes to the $\left(\frac{0}{1}, \frac{1}{0}\right)$-framing of the corresponding tangle sphere of $\mathcal{P}$. Thus the fact that $S^{3}=L\left(\alpha, \beta, \gamma, \delta, \frac{1}{2}\right)$ becomes the fact that the unknot is $\mathcal{P}\left(\alpha^{\prime}, \beta^{\prime}, \gamma^{\prime}, \delta^{\prime}, \frac{2}{1}\right)$. The condition that $\Delta\left(\chi, \frac{0}{1}\right) \geq 2$ for $\chi \in\{\alpha, \beta, \gamma, \delta\}$, becomes the condition that $\Delta\left(\chi, \frac{1}{0}\right) \geq 2$ for $\chi \in\left\{\alpha^{\prime}, \beta^{\prime}, \gamma^{\prime}, \delta^{\prime}\right\}$. This latter condition is equivalent to $\mathcal{P}\left(\alpha^{\prime}, \beta^{\prime}, \gamma^{\prime}, \delta^{\prime}, \frac{0}{1}\right)$ being the union of prime tangles. By Proposition 6.2, $\mathcal{P}\left(\alpha^{\prime}, \beta^{\prime}, \gamma^{\prime}, \delta^{\prime}, *\right)=B(\ell, m, n, p)$ for some $\ell, m, n, p$.

Thus $E(K)=L(\alpha, \beta, \gamma, \delta, *)$ is the double branched cover of $B(\ell, m, n, p)$. That is, $K=k(\ell, m, n, p)$ and the toroidal surgery is the corresponding halfintegral surgery by $[6]$.
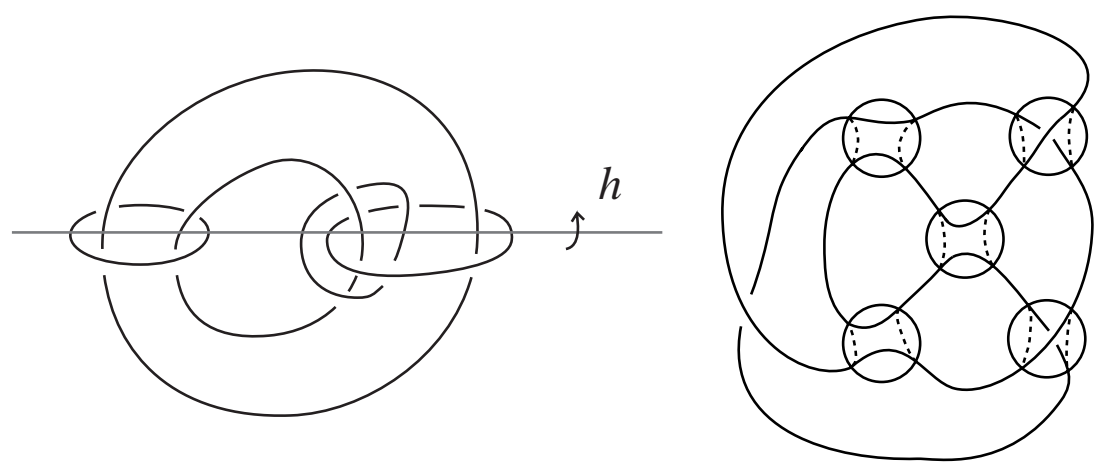

Figure 6.5.

\section{Tangle insertions in $\mathcal{Q}$ and their double branched covers.}

Sections 7 and 8 are devoted to lemmas supporting the proof of Proposition 6.1. In particular, let $\mathcal{Q}$ be the tangle of Proposition 6.1, and $\mathcal{Q}(\alpha, \beta, \gamma, \delta)$ the given tangle filling. In this section we do a series of computations describing the double branched covers of certain tangle fillings of $\mathcal{Q}$. 
These, combined with certain Dehn filling theorems, will lead in Section 8 to restrictions on the tangle slopes $\alpha, \beta, \gamma, \delta$.

Let $F$ be a surface, possibly with boundary. Let $e_{1}, e_{2}, \ldots, e_{n}$ be nonnegative integers. Define $F\left(e_{1}, e_{2}, \ldots, e_{n}\right)$ to be any manifold obtained by doing Dehn surgery on $n$ circle fibers of $F \times S^{1}$ so that the surgery slope on the $i^{t h}$ circle fiber has distance $e_{i}$ from its meridian. If $e_{i}>0$ for each $i$, then any $F\left(e_{1}, \ldots, e_{n}\right)$ is a Seifert fiber space over $F$ with exceptional fibers of orders those $e_{i}$ 's that are $>1$. If some $e_{i}=0$, then any $F\left(e_{1}, e_{2}, \ldots, e_{n}\right)$ is either a lens space, or $S^{3}$, or a solid torus, or is reducible.

In this paper $F$ will be either $A^{2}$, an annulus, $D^{2}$, a disk, or $S^{2}$, a 2sphere.

A manifold of the form $A^{2}(q)$ with $q \geq 2$ is called a cable space.

Recall that $\tilde{\mathcal{Q}}(\alpha, \beta, \gamma, \delta)$ denotes the double branched cover of $S^{3}$ along $\mathcal{Q}(\alpha, \beta, \gamma, \delta)$.

Lemma 7.1. $\tilde{\mathcal{Q}}\left(\alpha, \beta, \gamma, \frac{0}{1}\right)=S^{2}(a, b, c)$ where $a=\Delta\left(\alpha, \frac{1}{0}\right), b=\Delta\left(\beta, \frac{1}{2}\right)$, $c=\Delta\left(\gamma, \frac{1}{1}\right)$.

Proof. Figure 7.1 shows that $\mathcal{Q}\left(\alpha, \beta, \gamma, \frac{0}{1}\right)$ is a Montesinos link of length 3 . Thus the double branched cover $\tilde{\mathcal{Q}}\left(\alpha, \beta, \gamma, \frac{0}{1}\right)$ of $\mathcal{Q}\left(\alpha, \beta, \gamma, \frac{0}{1}\right)$ will be $S^{2}(a, b, c)$. In Figure 7.1 one sees that the slope of the Seifert fiber on $A$ corresponds to the tangle slope $1 / 0$, on $B$ to the tangle slope $1 / 2$, and on $C$ to the tangle slope $1 / 1$. Thus $a=\Delta\left(\alpha, \frac{1}{0}\right), b=\Delta\left(\beta, \frac{1}{2}\right)$, and $c=\Delta\left(\gamma, \frac{1}{1}\right)$.
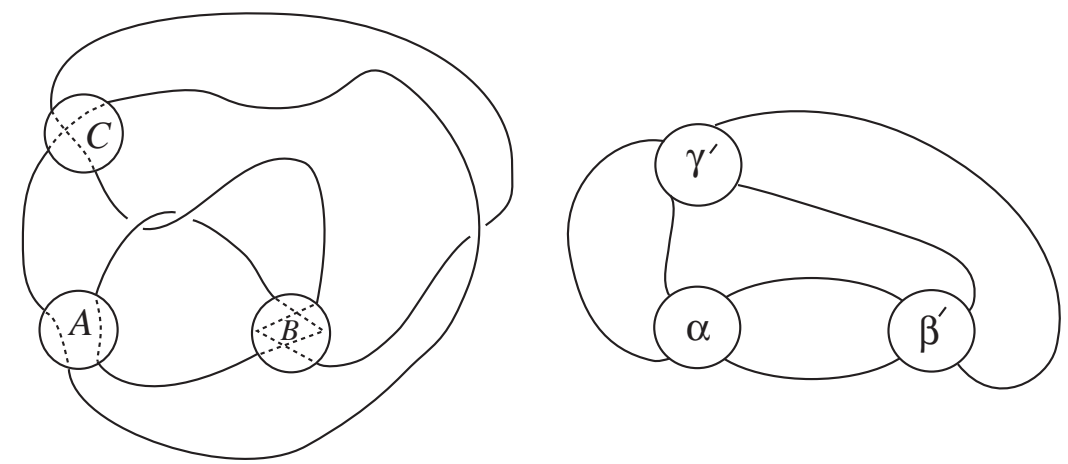

Figure 7.1.

Lemma 7.2. $\tilde{\mathcal{Q}}\left(\alpha, \beta, \gamma, \frac{1}{1}\right)=S^{2}(a, b, c)$ where $a=\Delta\left(\alpha, \frac{1}{2}\right), b=\Delta\left(\beta, \frac{1}{0}\right)$, $c=\Delta\left(\gamma, \frac{0}{1}\right)$. 
Proof. Similar to the proof of Lemma 7.1.

Lemma 7.3. $\tilde{\mathcal{Q}}\left(\alpha, \beta, \gamma, \frac{1}{0}\right)=X_{1} \bigcup_{T} X_{2}$ where $X_{1}=D^{2}(a, b), \quad X_{2}=$ $D^{2}(2, c)$, and $T$ is a 2-torus. Furthermore $a=\Delta\left(\alpha, \frac{0}{1}\right), b=\Delta\left(\beta, \frac{1}{1}\right)$, $c=\Delta\left(\gamma, \frac{1}{0}\right)$. Finally the given Seifert fibers of $X_{1}$ and $X_{2}$ intersect once on $T$.

Proof. Figure 7.2 decomposes $\mathcal{Q}\left(\alpha, \beta, \gamma, \frac{1}{0}\right)$ along a tangle sphere $P$. Each side of $P$ is then further decomposed as a tangle sum by the disks $Q_{1}$ and $Q_{2}$. Thus $\tilde{\mathcal{Q}}\left(\alpha, \beta, \gamma, \frac{1}{0}\right)$ decomposes along a 2 -torus $T$ into two Seifert fiber spaces $D^{2}(a, b), D^{2}(2, c)$ where the Seifert fibers intersect once on $T$. The slope of the Seifert fiber on $A$ is $0 / 1$, on $B$ is $1 / 1$, and on $C$ is $1 / 0$ (pictured in the top half of Figure 7.2). Thus $a=\Delta\left(\alpha, \frac{0}{1}\right), b=\Delta\left(\beta, \frac{1}{1}\right)$, and $c=\Delta\left(\gamma, \frac{1}{0}\right)$.

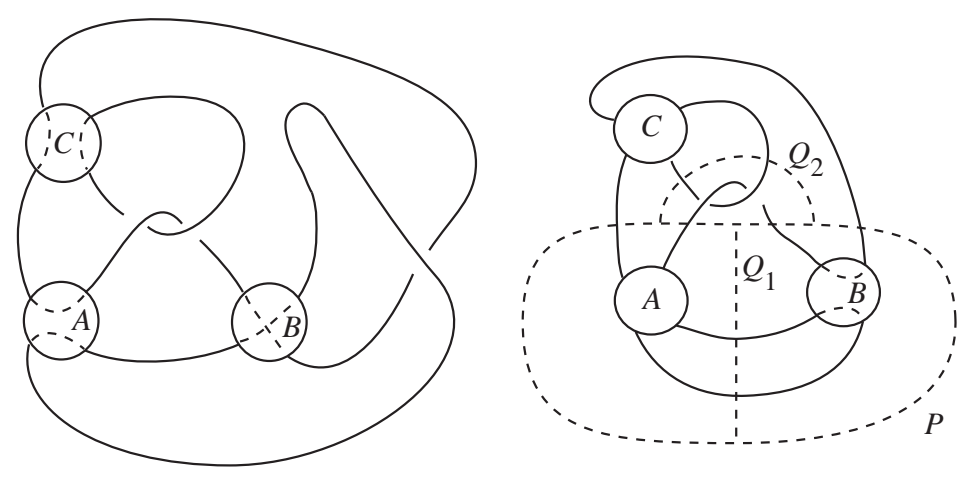

Figure 7.2.

Lemma 7.4. $\tilde{\mathcal{Q}}\left(\alpha, \beta, \gamma, \frac{1}{2}\right)=X_{1} \bigcup_{T} X_{2}$ where $X_{1}=D^{2}(a, b), X_{2}=D^{2}(2, c)$ and $T$ is a 2-torus. Furthermore $a=\Delta\left(\alpha, \frac{1}{1}\right), b=\Delta\left(\beta, \frac{0}{1}\right), c=\Delta\left(\gamma, \frac{1}{2}\right)$. Finally the given Seifert fibers of $X_{1}$ and $X_{2}$ intersect once on $T$.

Proof. Follow the proof of Lemma 7.3 using Figure 7.3.

Lemma 7.5. $\tilde{\mathcal{Q}}\left(\frac{0}{1}, \beta, \gamma, *\right)=D^{2}(b, c)$ where $b=\Delta\left(\beta, \frac{1}{1}\right), c=\Delta\left(\gamma, \frac{1}{2}\right)$.

Proof. Figure 7.4 writes the tangle $\mathcal{Q}\left(\frac{0}{1}, \beta, \gamma, *\right)$ as a tangle sum of $B$ and 

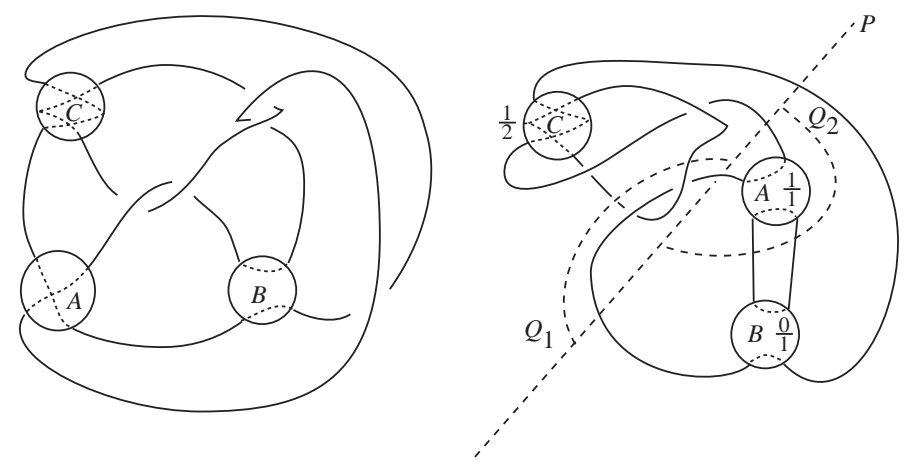

Figure 7.3.

$C$ along the disk $Q$. Thus $\tilde{\mathcal{Q}}\left(\frac{0}{1}, \beta, \gamma, *\right)=D^{2}(b, c)$. The Seifert fiber on $B$ corresponds to the tangle slope $1 / 1$, and on $C$ corresponds to $1 / 2$.
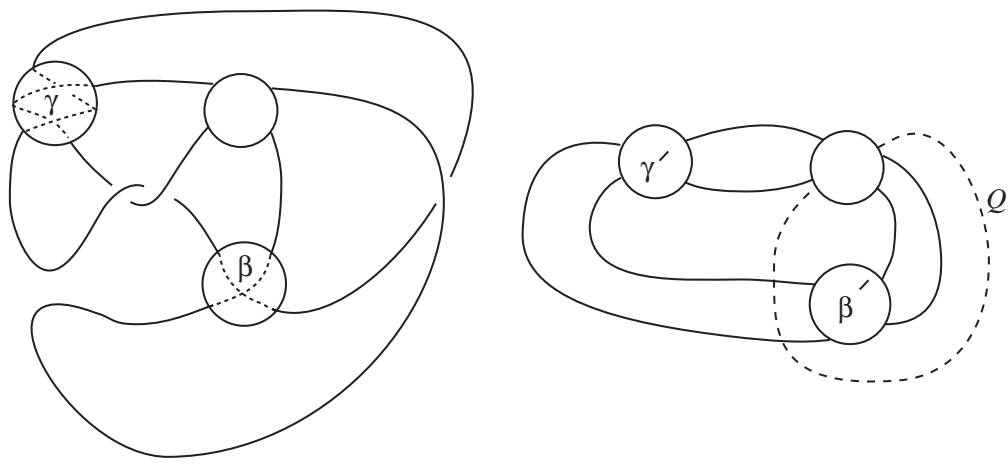

Figure 7.4.

Lemma 7.6. $\tilde{\mathcal{Q}}\left(\frac{1}{1}, \beta, \gamma, *\right)=D^{2}(b, c)$ where $b=\Delta\left(\beta, \frac{0}{1}\right), c=\Delta\left(\gamma, \frac{1}{0}\right)$.

Proof. Similar to the proof of Lemma 7.5.

Lemma 7.7. $\tilde{\mathcal{Q}}\left(\frac{1}{0}, \beta, \gamma, *\right)=X_{1} \bigcup_{T} X_{2}$ where $X_{1}=D^{2}(2, b), X_{2}=A^{2}(c)$ and $b=\Delta\left(\beta, \frac{1}{0}\right), c=\Delta\left(\gamma, \frac{1}{1}\right)$.

Proof. Figure 7.5 decomposes the tangle $\mathcal{Q}\left(\frac{1}{0}, \beta, \gamma, *\right)$ along the tangle sphere $P$. Each side of $P$ is then further decomposed as a tangle sum by the disks 
$Q_{1}$ and $Q_{2}$. Thus $\tilde{\mathcal{Q}}\left(\frac{1}{0}, \beta, \gamma, *\right)$ decomposes along a 2-torus into $D^{2}(2, b)$ and $A^{2}(c)$. Figure 7.5 also identifies the Seifert fibers on $B$ and $C$, giving $b=\Delta\left(\beta, \frac{1}{0}\right), c=\Delta\left(\gamma, \frac{1}{1}\right)$.
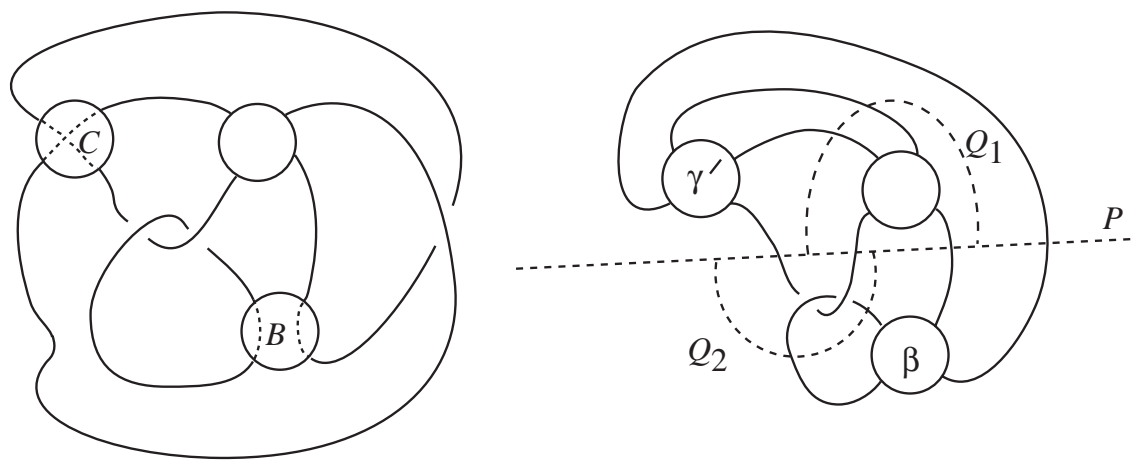

Figure 7.5.

Lemma 7.8. $\tilde{\mathcal{Q}}\left(\frac{1}{2}, \beta, \gamma, *\right)=X_{1} \bigcup_{T} X_{2}$ where $X_{1}=D^{2}(2, b), X_{2}=A^{2}(c)$ and $b=\Delta\left(\beta, \frac{1}{2}\right), c=\Delta\left(\gamma, \frac{0}{1}\right)$. Furthermore the Seifert fibers of $X_{1}$ and $X_{2}$ intersect once on $T$.

Proof. Follow the proof of Lemma 7.7, using Figure 7.6.
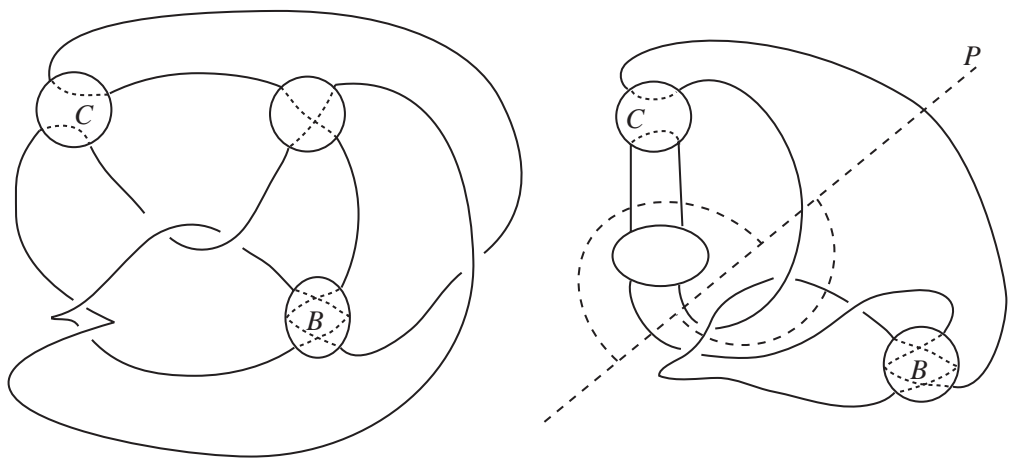

Figure 7.6.

Lemma 7.9. $\tilde{\mathcal{Q}}\left(\alpha, \frac{0}{1}, \gamma, *\right)=D^{2}(a, c)$ where $a=\Delta\left(\alpha, \frac{1}{1}\right), c=\Delta\left(\gamma, \frac{1}{0}\right)$. 
Proof. Follow the proof of Lemma 7.5, using Figure 7.7.

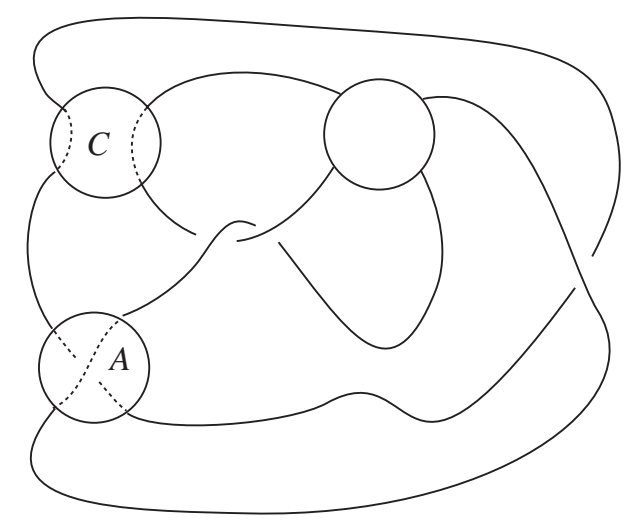

Figure 7.7.

Lemma 7.10. $\tilde{\mathcal{Q}}\left(\alpha, \frac{1}{1}, \gamma, *\right)=D^{2}(a, c)$ where $a=\Delta\left(\alpha, \frac{0}{1}\right), c=\Delta\left(\gamma, \frac{1}{2}\right)$.

Proof. Similar to Lemma 7.9.

Lemma 7.11. $\tilde{\mathcal{Q}}\left(\alpha, \frac{1}{0}, \gamma, *\right)=X_{1} \bigcup_{T} X_{2}$ where $X_{1}=D^{2}(2, a), X_{2}=A^{2}(c)$ and $T$ is the common 2-torus boundary of $X_{1}, X_{2}$. Furthermore $a=\Delta\left(\alpha, \frac{1}{0}\right)$, $c=\Delta\left(\gamma, \frac{0}{1}\right)$.

Proof. Follow the proof of Lemma 7.7 using Figure 7.8.

Lemma 7.12. $\tilde{\mathcal{Q}}\left(\alpha, \frac{1}{2}, \gamma, *\right)=X_{1} \bigcup_{T} X_{2}$ where $X_{1}=D^{2}(2, a), X_{2}=A^{2}(c)$ and $T$ is the common 2-torus boundary of $X_{1}, X_{2}$. Furthermore $a=\Delta\left(\alpha, \frac{1}{2}\right)$, $c=\Delta\left(\gamma, \frac{1}{1}\right)$. The Seifert fibers of $X_{1}$ and $X_{2}$ intersect once on $T$.

Proof. Same as Lemma 7.3, using Figure 7.9.

Lemma 7.13. $\tilde{\mathcal{Q}}\left(\alpha, \beta, \frac{0}{1}, \frac{1}{2}\right)$ is a lens space, $S^{3}$, or $S^{2} \times S^{1}$. 


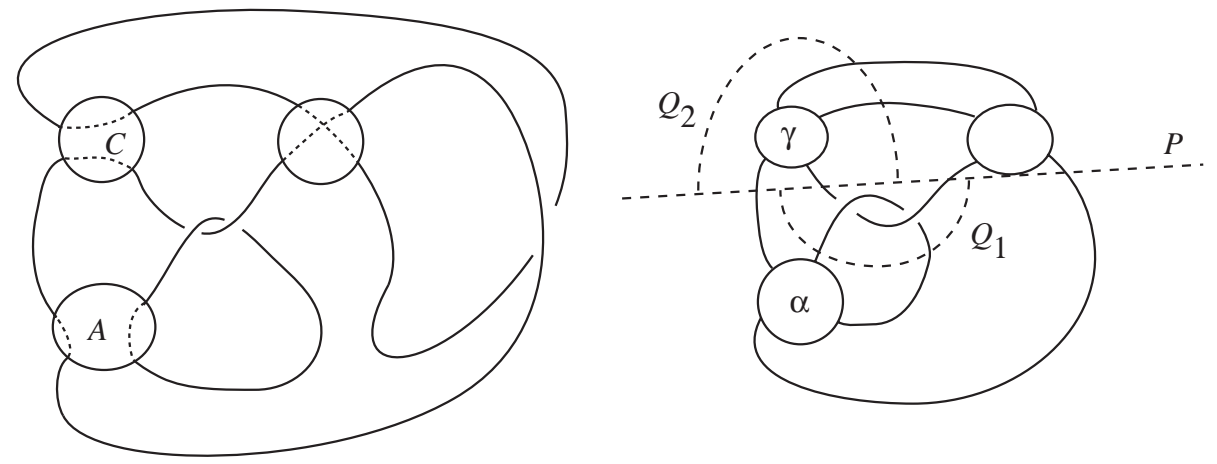

Figure 7.8.
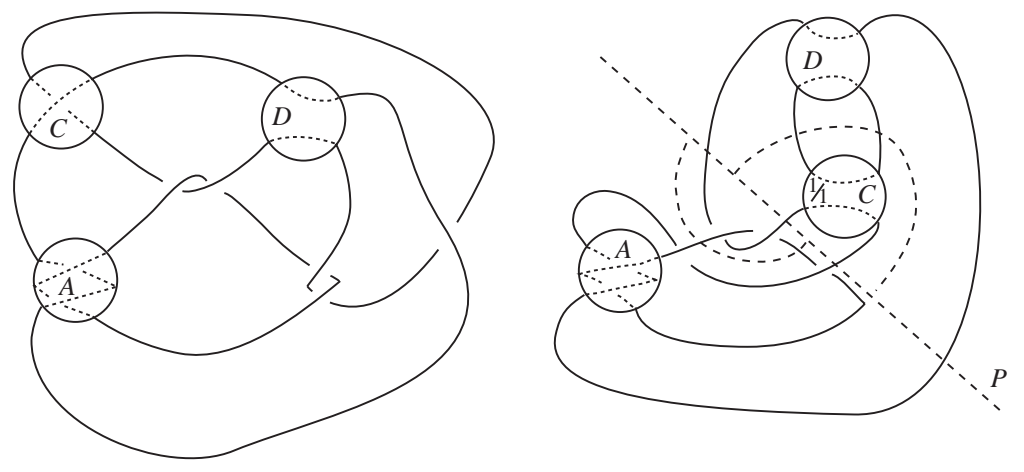

Figure 7.9.

Proof. Figure 7.10 shows that $\mathcal{Q}\left(\alpha, \beta, \frac{0}{1}, \frac{1}{2}\right)$ is the union of two rational tangles.

Lemma 7.14. $\tilde{\mathcal{Q}}\left(\alpha, \beta, \frac{1}{1}, \frac{1}{2}\right)$ is a lens space, $S^{3}$, or $S^{2} \times S^{1}$.

Proof. Similar to Lemma 7.13.

\section{Applications of Dehn filling restrictions.}

In this section we prove a series of lemmas leading to the proof of Proposition 6.1 in Section 6. Thus we assume we are in the context of Proposition 6.1. In particular, $\mathcal{Q}(\alpha, \beta, \gamma, \delta)$ is the unknot.

Recall that $\tilde{\mathcal{Q}}$ is the double branched cover of $\mathcal{Q}$. Since $\mathcal{Q}(\alpha, \beta, \gamma, \delta)$ is 


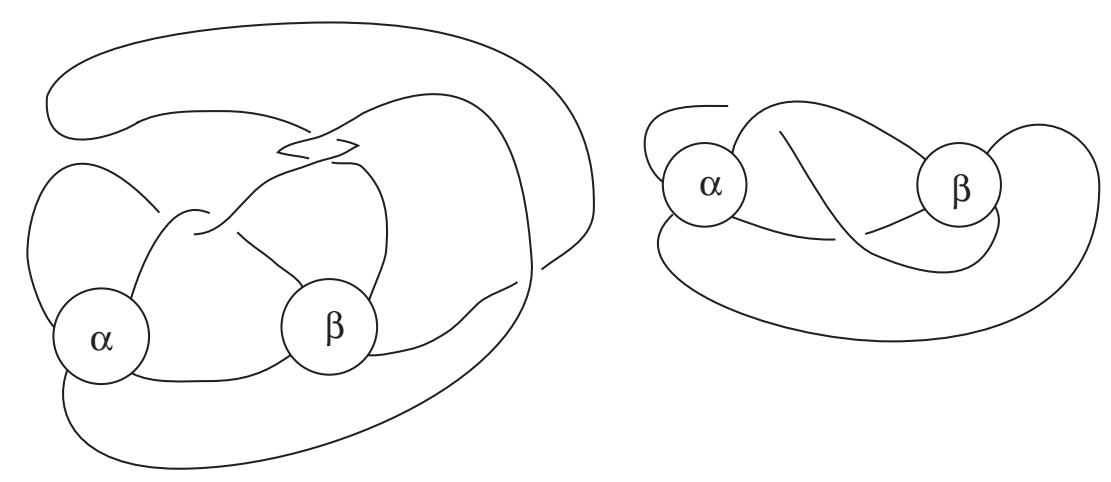

Figure 7.10 .

the unknot, $\tilde{\mathcal{Q}}(\alpha, \beta, \gamma, \delta)$ is the 3 -sphere. Let $K_{A}, K_{B}, K_{C}, K_{D}$ be the knots in $\tilde{\mathcal{Q}}(\alpha, \beta, \gamma, \delta)=S^{3}$ corresponding to the fillings $\alpha, \beta, \gamma, \delta$ (resp.) of $\tilde{\mathcal{Q}}$ (i.e., they are the cores of the solid tori $\tilde{R}(\alpha), \tilde{R}(\beta), \tilde{R}(\gamma), \tilde{R}(\delta))$.

In Section 7, we described certain Dehn fillings of $\tilde{\mathcal{Q}}$. Here we will combine those computations with theorems about Dehn fillings to place restrictions on $\alpha, \beta, \gamma, \delta$.

First we put together some results describing restrictions on the slopes of certain Dehn fillings of a 3-manifold.

Lemma 8.1. Let $M$ be an irreducible 3-manifold with $\partial M$ a torus. Let $\alpha, \beta$ be slopes on $\partial M$ such that $M(\alpha)$ is reducible and $M(\beta)$ is either reducible, a lens space, or $S^{3}$. Then $\Delta(\alpha, \beta) \leq 1$.

Proof. If $M(\beta)$ is reducible, this follows from [14]. If $M(\beta)$ is a lens space or $S^{3}$, then [3] shows that either $\Delta(\alpha, \beta) \leq 1$ or $M=D^{2}(a, b)$ with $a, b \geq 2$. We assume the latter. Since $M(\alpha)$ is reducible, $\alpha$ must be the Seifert fiber of $M$ ([10, Corollary 7.4]). Since $M(\beta)$ is a lens space (or $\left.S^{3}\right), \Delta(\alpha, \beta) \leq 1$ ([10, Corollary 7.4]).

Lemma 8.2. Let $M$ be a 3-manifold, with $\partial M$ consisting of two tori, and let $\alpha, \beta$ be slopes on one component of $\partial M$ such that $M(\alpha)=S^{1} \times D^{2}$, $M(\beta)=D^{2}(m, n)$ with $m, n \geq 1$. Then either

(1) $\Delta(\alpha, \beta) \leq 1$ or

(2) $M$ is the exterior of a $(p, q)$-cable, $q \geq 2$, of a knot $K_{0}$ in $M(\alpha)=$ $S^{1} \times D^{2}$. Let $\rho$ be the slope of the cabling annulus on $K_{0}$. Then either 
(2a) $K_{0}$ is isotopic to the core of $M(\alpha)$ and $M=A^{2}(q) . M(\chi)$ is boundary compressible iff $\Delta(\chi, \rho) \leq 1$ and $M(\chi)$ is reducible iff $\chi=\rho$.

(2b) $K_{0}$ is not isotopic to the core of $M(\alpha)$. In this case $M(\chi)$ contains an embedded essential torus if $\Delta(\chi, \rho) \geq 2$, and $M(\rho)$ is reducible.

(3) $M=T^{2} \times I$. In this case $M(\chi)=S^{1} \times D^{2}$ for any slope $\chi$.

Remark. In case (2b), if $M(\chi)$ is atoroidal and irreducible then $\Delta(\chi, \rho)=1$. In particular, if there are three slopes $\chi$ with pairwise distance 1 (e.g., $\left.\frac{0}{1}, \frac{1}{1}, \frac{1}{0}\right)$, and such that $M(\chi)=D^{2}(m, n)$ with $m, n \geq 1$, then $(2 \mathrm{~b})$ is impossible.

Proof. Let $\partial_{1} M$ denote the component of $\partial M$ on which the slopes $\alpha, \beta$ are defined, and let $\partial_{2} M$ be the remaining component. Let $M(r, s)$ denote the $r$-filling of $\partial_{1} M$ and $s$-filling of $\partial_{2} M$. Then there are infinitely many different slopes $r_{i}, i \in \mathbb{Z}$, such that $M\left(\beta, r_{i}\right)=S^{2}(m, n)$ and $\Delta\left(r_{i}, r_{j}\right)=|i-j|$. At the same time, for each $i, M\left(\alpha, r_{i}\right)=S^{2}(a, b)$ for some $a, b$.

Assume $\Delta(\alpha, \beta) \geq 2$. Then, for each $i$, the above observations coupled with [4, Cyclic Surgery Theorem] or with Lemma 8.1 above imply that $M\left(*, r_{i}\right)$ is either reducible or a Seifert fiber space. By the Gromov-Thurston $2 \pi$-Theorem [2], the interior of $M$ cannot be hyperbolic of finite volume. Thus either

(i) $M$ contains an essential 2-sphere; or

(ii) $M$ contains an essential 2-torus; or

(iii) $M=A^{2}(q)$ for some $q \geq 1$.

Case (iii) corresponds to (2a), using [10, Lemma 7.2] or to case (3). Since $M(\alpha), M(\beta)$ are irreducible, [12] implies that (i) cannot hold. Thus we are left to consider case (ii). Let $T$ be an essential torus in $M$. Since $T$ compresses in $M(\alpha), M(\beta)$, and $\Delta(\alpha, \beta) \geq 2$, [4, Theorem 2.4.4] implies that $T$ cobounds with $\partial_{1} M$ a cable space. That is, $M=N_{1} \bigcup_{T} N_{2}$ where $\partial_{i} M \subset \partial N_{i}, i=1,2$, and $N_{1}=A^{2}(q)$ for some $q \geq 2$. [10, Lemma 7.3] shows that $M$ is the exterior of a $(p, q)$-cable of a knot $K_{0}$ in $M(\alpha)$ and that $(2 \mathrm{~b})$ holds.

Lemma 8.3. If $K_{D}$ is an unknot or torus knot then either $\Delta\left(\gamma, \frac{1}{2}\right) \leq 1$ or one of $\alpha, \beta$ is $1 / 2$. 
Proof. Assume that $K_{D}$ is an unknot or torus knot, $\Delta\left(\gamma, \frac{1}{2}\right) \geq 2$, and $\alpha \neq$ $1 / 2 \neq \beta$. No surgery on $K_{D}$ contains a separating incompressible torus. Since $\Delta\left(\gamma, \frac{1}{0}\right) \geq 2$, Lemma 7.3 implies

$$
\Delta\left(\alpha, \frac{0}{1}\right) \leq 1 \text { or } \Delta\left(\beta, \frac{1}{1}\right) \leq 1 .
$$

Since $\Delta\left(\gamma, \frac{1}{2}\right) \geq 2$, Lemma 7.4 says that

$$
\Delta\left(\alpha, \frac{1}{1}\right) \leq 1 \text { or } \Delta\left(\beta, \frac{0}{1}\right) \leq 1 .
$$

Note that if $\Delta\left(r, \frac{0}{1}\right) \leq 1, \Delta\left(r, \frac{1}{1}\right) \leq 1$, then $r \in\left\{\frac{0}{1}, \frac{1}{1}, \frac{1}{2}, \frac{1}{0}\right\}$. Since $\Delta\left(\alpha, \frac{1}{0}\right)$, $\Delta\left(\beta, \frac{1}{0}\right) \geq 2,(8.1)$ and (8.2) give two possibilities:

Case $(\mathrm{A}): \quad \Delta\left(\alpha, \frac{0}{1}\right)=1$ and $\Delta\left(\beta, \frac{0}{1}\right)=1$.

Case $(\mathrm{B}): \quad \Delta\left(\alpha, \frac{1}{1}\right)=1$ and $\Delta\left(\beta, \frac{1}{1}\right)=1$.

Case (A): By Lemma 7.6, $\tilde{\mathcal{Q}}\left(\frac{1}{1}, \beta, \gamma, *\right)=S^{1} \times D^{2}$. By assumption $\tilde{\mathcal{Q}}(\alpha, \beta, \gamma, *)=D^{2}(m, n)$ for some $m, n \geq 1$. If $\Delta\left(\frac{1}{1}, \alpha\right) \leq 1$, then $\alpha=1 / 2$. So we assume $\Delta\left(\alpha, \frac{1}{1}\right) \geq 2$. By Lemma $8.2, M=\tilde{\mathcal{Q}}(*, \beta, \gamma, *)$ is as in Lemma $8.2(2 \mathrm{a})$ or $(2 \mathrm{~b})$. (Lemma 7.7 rules out (3).)

Assume we are as in Lemma $8.2(2 \mathrm{~b})$. Recall that $\rho$ is the slope of the cabling annulus in $M$. By Lemma $8.2(2 \mathrm{~b}), \Delta\left(\frac{1}{1}, \rho\right)=1$. Lemma 7.5 says that $\tilde{\mathcal{Q}}\left(\frac{0}{1}, \beta, \gamma, *\right)=D^{2}(b, c)$ with $b, c \geq 1$. Thus $\Delta\left(\frac{0}{1}, \rho\right)=1$. Together these say that $\rho \in\left\{\frac{1}{2}, \frac{1}{0}\right\}$. Now $\tilde{\mathcal{Q}}(\rho, \beta, \gamma, *)$ is reducible. But Lemma 7.7 says that $\tilde{\mathcal{Q}}\left(\frac{1}{0}, \beta, \gamma, *\right)$ is irreducible $\left(\Delta\left(\beta, \frac{1}{0}\right), \Delta\left(\gamma, \frac{1}{0}\right) \geq 2\right)$. Lemma 7.8 says that $\tilde{\mathcal{Q}}\left(\frac{1}{2}, \beta, \gamma, *\right)$ is irreducible. (Since $\beta \neq \frac{1}{2}, \gamma \neq \frac{0}{1}$, if $X_{1} \bigcup_{T} X_{2}$ is reducible, then $X_{1}=D^{2}(2,1)$ and the meridian disk of $X_{1}$ is attached to the Seifert fiber of $X_{2}$. But the Seifert fiber of $X_{2}$ intersects the Seifert fiber of $X_{1}$ once by Lemma 7.8. The boundary of the meridian disk in $D^{2}(2,1)$ has intersection number two with its Seifert fiber on $\partial D^{2}(2,1)$.) This contradicts the assumption that $M$ is as in Lemma $8.2(2 \mathrm{~b})$.

Thus $\tilde{\mathcal{Q}}(*, \beta, \gamma, *)=A^{2}(q)$ for $q \geq 2$. So $\tilde{\mathcal{Q}}\left(\frac{1}{0}, \beta, \gamma, *\right)$ is atoroidal. By Lemma $7.7\left(\Delta\left(\beta, \frac{1}{0}\right) \geq 2\right), \Delta\left(\gamma, \frac{1}{1}\right) \leq 1$.

By following a similar argument using Lemmas 7.9-7.12 (in place of 7.57.8) with $M=\tilde{\mathcal{Q}}(\alpha, *, \gamma, *)$, we conclude that $\Delta\left(\gamma, \frac{0}{1}\right) \leq 1$. (I.e., Lemma 7.10 says $\tilde{\mathcal{Q}}\left(\alpha, \frac{1}{1}, \gamma, *\right)=S^{1} \times D^{2}$. Then Lemma 8.2 says $M=\tilde{\mathcal{Q}}(\alpha, *, \gamma, *)$ is as in Lemma $8.2(2 \mathrm{a})$ or $(2 \mathrm{~b})$.)

Since $\Delta\left(\gamma, \frac{1}{0}\right) \geq 2$ and $\Delta\left(\gamma, \frac{1}{2}\right) \geq 2$, this is a contradiction. $\square$ (Case (A))

Case (B): The argument follows the same sequence as in Case (A). Lemma 7.5 implies that $\tilde{\mathcal{Q}}\left(\frac{0}{1}, \beta, \gamma, *\right)=S^{1} \times D^{2}$. Since $\Delta\left(\alpha, \frac{0}{1}\right) \geq 2$, Lemma 8.2 implies that $M=\tilde{\mathcal{Q}}(*, \beta, \gamma, *)$ is as in Lemma $8.2(2 \mathrm{a})$ or $(2 \mathrm{~b})$. 
We rule out Lemma $8.2(2 \mathrm{~b})$ as follows. The above says that $\Delta\left(\frac{0}{1}, \rho\right)=1$. Lemma 7.6 says that $\Delta\left(\frac{1}{1}, \rho\right)=1$. Thus $\rho \in\left\{\frac{1}{2}, \frac{1}{0}\right\}$. However, Lemmas 7.7 and 7.8 imply that both $\tilde{\mathcal{Q}}\left(\frac{1}{0}, \beta, \gamma, *\right)$ and $\tilde{\mathcal{Q}}\left(\frac{1}{2}, \beta, \gamma, *\right)$ are irreducible. This contradicts the fact that $\tilde{\mathcal{Q}}(\rho, \beta, \gamma, *)$ is reducible.

So $\tilde{\mathcal{Q}}(*, \beta, \gamma, *)=A^{2}(q)$ for $q \geq 2($ Lemma $8.2(2 \mathrm{a}))$. Thus $\tilde{\mathcal{Q}}\left(\frac{1}{0}, \beta, \gamma, *\right)$ is atoroidal. Lemma 7.7 then says $\Delta\left(\gamma, \frac{1}{1}\right) \leq 1$.

Arguing in the same way using Lemmas 7.9-7.12 in place of 7.5-7.8 (and $M=\tilde{\mathcal{Q}}(\alpha, *, \gamma, *))$ shows that $\Delta\left(\gamma, \frac{0}{1}\right) \leq 1$. Thus, as in Case $(\mathrm{A})$, we get a contradiction.

$\square$ (Case (B))

Proposition 8.4. If $\Delta\left(\gamma, \frac{1}{0}\right)=2$ then one of $\alpha, \beta$, or $\gamma$ is $1 / 2$.

Proof. Assume, for contradiction, that $\Delta\left(\gamma, \frac{1}{0}\right)=2$ and neither $\alpha, \beta$, nor $\gamma$ is $1 / 2$.

By Lemma $7.3, \tilde{\mathcal{Q}}\left(\alpha, \beta, \gamma, \frac{1}{0}\right)$ contains $D^{2}(2,2)$, hence a Klein bottle. Since $\Delta\left(\delta, \frac{1}{0}\right) \geq 2, K_{D}$ must be the unknot, a torus knot, or a cable knot [22, Theorem 1.1]. Since $\Delta\left(\gamma, \frac{1}{0}\right)=2, \Delta\left(\gamma, \frac{1}{2}\right)$ is even. Hence $\Delta\left(\gamma, \frac{1}{2}\right) \geq 2$. But then, by Lemma 8.3, we may assume that $K_{D}$ is a non-trivial cable on a knot $K_{0}$ in $S^{3}$. Let $\rho$ be the slope of the cabling annulus on $K_{D}$. Lemmas 7.1 and 7.2 say that $\tilde{\mathcal{Q}}\left(\alpha, \beta, \gamma, \frac{0}{1}\right)$ and $\tilde{\mathcal{Q}}\left(\alpha, \beta, \gamma, \frac{1}{1}\right)$ are irreducible and contain no separating incompressible tori. Thus by [10, Lemma 7.2] $\Delta\left(\rho, \frac{0}{1}\right)=\Delta\left(\rho, \frac{1}{1}\right)=1$. Thus $\rho \in\left\{\frac{1}{0}, \frac{1}{2}\right\}$. By [10, Lemma 7.2] $\tilde{\mathcal{Q}}(\alpha, \beta, \gamma, \rho)$ will be reducible. But this is contradicted by Claims 1 and 2 below.

Claim 1. $\tilde{\mathcal{Q}}\left(\alpha, \beta, \gamma, \frac{1}{2}\right)$ is irreducible.

Proof. Since $\Delta\left(\chi, \frac{1}{0}\right) \geq 2$ for $\chi \in\{\alpha, \beta\}$, and $\Delta\left(\gamma, \frac{1}{2}\right) \geq 2$, Lemma 7.4 implies that if $\tilde{\mathcal{Q}}\left(\alpha, \beta, \gamma, \frac{1}{2}\right)$ is reducible, it must be the filling of $D^{2}(2, c)$ along its fiber slope. Thus $H_{1}\left(\tilde{\mathcal{Q}}\left(\alpha, \beta, \gamma, \frac{1}{2}\right)\right)=\mathbb{Z} / 2 \mathbb{Z} \oplus \mathbb{Z} / c \mathbb{Z}$. But $c=\Delta\left(\gamma, \frac{1}{2}\right)$ is even, hence $H_{1}\left(\tilde{\mathcal{Q}}\left(\alpha, \beta, \gamma, \frac{1}{2}\right)\right)$ is not generated by a single element. This contradicts that $\tilde{\mathcal{Q}}\left(\alpha, \beta, \gamma, \frac{1}{2}\right)$ is a Dehn surgery on a knot in $S^{3}$. $\square$ (Claim 1)

Claim 2. $\tilde{\mathcal{Q}}\left(\alpha, \beta, \gamma, \frac{1}{0}\right)$ is irreducible.

Proof. As in Claim 1, since $\alpha \neq \frac{0}{1}, \beta \neq \frac{1}{1}$, and $\Delta\left(\gamma, \frac{1}{0}\right)=2$, Lemma 7.3 implies that the only way $\tilde{\mathcal{Q}}\left(\alpha, \beta, \gamma, \frac{1}{0}\right)$ is reducible is if it is the filling of $D^{2}(2,2)$ along its Seifert fiber. But the homology of the resulting connected sum is not generated by a single element. This contradicts the fact that $\tilde{\mathcal{Q}}\left(\alpha, \beta, \gamma, \frac{1}{0}\right)$ is a Dehn surgery on a knot in $S^{3}$. 
Corollary 8.5. Assume $\mathcal{Q}(\alpha, \beta, \gamma, \delta)$ is as in Proposition 6.1. If $\Delta\left(\chi, \frac{1}{0}\right)=2$ for some $\chi \in\{\alpha, \beta, \gamma, \delta\}$ then $\frac{1}{2} \in\{\alpha, \beta, \gamma, \delta\}$.

Proof. Apply a symmetry taking $\chi$ to $\gamma$, then Proposition 8.4.

Proposition 8.6. Let $\mathcal{Q}(\alpha, \beta, \gamma, \delta)$ be as in Proposition 6.1. If $\Delta\left(\delta, \frac{1}{0}\right) \geq 3$ and $\Delta\left(\gamma, \frac{1}{0}\right) \geq 2$ then either $\gamma=\frac{1}{2}$, or $\Delta\left(\alpha, \frac{0}{1}\right) \leq 1$, or $\Delta\left(\beta, \frac{1}{1}\right) \leq 1$.

Proof. We assume that $\Delta\left(\alpha, \frac{0}{1}\right)>1$ and $\Delta\left(\beta, \frac{1}{1}\right)>1$, then show that $\gamma=$ $1 / 2$.

By Lemma 7.3 and the distance assumptions, $\tilde{\mathcal{Q}}\left(\alpha, \beta, \gamma, \frac{1}{0}\right)=X_{1} \bigcup_{T} X_{2}$ where $X_{i}=D^{2}\left(r_{i}, s_{i}\right)$ for some $r_{i}, s_{i} \geq 2$ and $i=1,2$. In particular, $\tilde{\mathcal{Q}}\left(\alpha, \beta, \gamma, \frac{1}{0}\right)$ is irreducible and $T$ is incompressible. Furthermore, $T$ is the unique incompressible torus in $\tilde{\mathcal{Q}}\left(\alpha, \beta, \gamma, \frac{1}{0}\right)$ up to isotopy. Since $\Delta\left(\delta, \frac{1}{0}\right) \geq 3$, [15, Theorem 1.1] shows that $K_{D}$ (the core of the attached solid torus corresponding to the tangle $D)$ is a satellite of a knot $K$ in $S^{3}=\tilde{\mathcal{Q}}(\alpha, \beta, \gamma, \delta)$. Taking an "outermost" companion we may assume that $K$ is either

(1) hyperbolic; or

(2) a torus knot.

Let $V$ be a solid torus neighborhood of $K$ containing $K_{D}$.

Assume that $\partial V$ compresses in $V\left(K_{D}, \frac{1}{0}\right)$. Since $\Delta\left(\delta, \frac{1}{0}\right) \geq 3,[4$, Theorem 2.4.4] says that $K_{D}$ is the $(p, q)$-cable of the core of $V$ where $q \geq 2$ is the winding number of $K_{D}$ in $V$. Then [10, Lemma 7.2 and Corollary 7.3] show that $K_{D}\left(\frac{1}{0}\right)=K(r)$ with $\Delta\left(r, \mu_{K}\right)=q^{2} \Delta\left(\frac{1}{0}, \delta\right)>2$ where $\mu_{K}$ is the meridian of $K$. Since $K(r)=K_{D}\left(\frac{1}{0}\right)=\tilde{\mathcal{Q}}\left(\alpha, \beta, \gamma, \frac{1}{0}\right)$ contains an essential torus, $K$ cannot be a torus knot (Case (2)). On the other hand $K$ cannot be hyperbolic (Case (1)) by [15, Theorem 1.1]. This is a contradiction.

Thus we assume that $\partial V$ is incompressible in $\tilde{\mathcal{Q}}\left(\alpha, \beta, \gamma, \frac{1}{0}\right)$. Then $\partial V$ is isotopic to the essential torus $T$, implying that the exterior of $K$ must be $D^{2}\left(r_{i}, s_{i}\right)$ (i.e. we are in Case $(2)$ - that $K$ is a torus knot) and $V\left(K_{D}, \frac{1}{0}\right)$ must be $D^{2}\left(r_{j}, s_{j}\right)$ where $\{i, j\}=\{1,2\}$. Let $M$ be the exterior of $K_{D}$ in $V=V\left(K_{D}, \delta\right)$. Since $V\left(K_{D}, \delta\right)=S^{1} \times D^{2}$ and $V\left(K_{D}, \frac{1}{0}\right)=D^{2}\left(r_{i}, s_{i}\right)$, and since $\Delta\left(\delta, \frac{1}{0}\right) \geq 3$, Lemma 8.2 says that $K_{D}$ is the $(p, q)$-cable of $K_{0} \subset V \subset S^{3}$ where $q \geq 2$ is the winding number of $K_{D}$ in a neighborhood of $K_{0}$. Let $\rho$ be the slope of the cabling annulus on $K_{D}$. Since $K_{0}$ is non-trivial in $S^{3}$ ( $K_{D}$ is not a torus knot), [10, Corollary 7.3$]$ says that $K_{D}(\rho)$ contains an essential 2-sphere and that $K_{D}(\chi)$ contains an essential torus if $\Delta(\chi, \rho) \geq 2$. 
However Lemmas 7.1 and 7.2, along with the distance assumptions, say that $K_{D}\left(\frac{0}{1}\right), K_{D}\left(\frac{1}{1}\right)$ contain no essential 2-spheres or (embedded) tori. Thus $\Delta\left(\rho, \frac{0}{1}\right)=\Delta\left(\rho, \frac{1}{1}\right)=1$. Together, these imply that $\rho=\frac{1}{0}$ or $\frac{1}{2}$. Since $K_{D}(\delta)$ is the 3 -sphere, $\Delta(\rho, \delta)=1$. Hence $\rho \neq \frac{1}{0}$. Thus $\rho=\frac{1}{2}$ and $K_{D}\left(\frac{1}{2}\right)=$ $\tilde{\mathcal{Q}}\left(\alpha, \beta, \gamma, \frac{1}{2}\right)$ is reducible. By Lemma $7.13, \tilde{\mathcal{Q}}\left(\alpha, \beta, \frac{0}{1}, \frac{1}{2}\right)$ is a lens space. (Here we use the term "lens space" to include $S^{3}$ and $S^{2} \times S^{1}$.) By Lemma 7.14, $\tilde{\mathcal{Q}}\left(\alpha, \beta, \frac{1}{1}, \frac{1}{2}\right)$ is a lens space. By [12], $\tilde{\mathcal{Q}}\left(\alpha, \beta, *, \frac{1}{2}\right)$ is irreducible (otherwise one of $\tilde{\mathcal{Q}}\left(\alpha, \beta, \frac{0}{1}, \frac{1}{2}\right), \tilde{\mathcal{Q}}\left(\alpha, \beta, \frac{1}{1}, \frac{1}{2}\right)$ contains a separating essential 2-sphere). Thus Lemma 8.1 says that $\Delta\left(\gamma, \frac{0}{1}\right) \leq 1$ and $\Delta\left(\gamma, \frac{1}{1}\right) \leq 1$. Since $\Delta\left(\gamma, \frac{1}{0}\right) \geq 2$ by assumption, $\gamma=\frac{1}{2}$.

For convenience, in Theorems 8.7-8.9 we will use the term "lens space" to include $S^{3}$ and $S^{2} \times S^{1}$.

Theorem 8.7. If $\mathcal{Q}(\alpha, \beta, \gamma, \delta)$ is the unknot then we cannot have $\Delta\left(\chi, \frac{1}{0}\right) \geq$ 3 and $\Delta\left(\chi, \frac{0}{1}\right)=1$ for every $\chi \in\{\alpha, \beta, \gamma, \delta\}$.

Proof. Assume $\Delta\left(\chi, \frac{1}{0}\right) \geq 3, \Delta\left(\chi, \frac{0}{1}\right)=1$ for $\chi \in\{\alpha, \beta, \gamma, \delta\}$. These imply that $\Delta\left(\chi, \frac{1}{1}\right) \geq 2$. By Lemma $7.2, \tilde{\mathcal{Q}}\left(\alpha, \beta, \gamma, \frac{1}{1}\right)$ is a lens space. So by $[4$, Cyclic Surgery Theorem], $K_{D}$ is an unknot or torus knot. By Lemma 8.3 we have that $\Delta\left(\gamma, \frac{1}{2}\right)=1$. Since $\Delta\left(\gamma, \frac{0}{1}\right)=1, \gamma \in\left\{\frac{1}{1}, \frac{1}{3}\right\}$. As $\Delta\left(\gamma, \frac{1}{0}\right) \geq 3$, $\gamma=\frac{1}{3}$.

By symmetry we get that $\alpha=\beta=\gamma=\delta=1 / 3$. But $\mathcal{Q}\left(\frac{1}{3}, \frac{1}{3}, \frac{1}{3}, \frac{1}{3}\right)$ is a 2-component link.

Theorem 8.8. If $\mathcal{Q}(\alpha, \beta, \gamma, \delta)$ is the unknot then we cannot have $\Delta\left(\chi, \frac{1}{0}\right) \geq$ 3 and $\Delta\left(\chi, \frac{1}{1}\right)=1$ for every $\chi \in\{\alpha, \beta, \gamma, \delta\}$.

Proof. Assume $\Delta\left(\chi, \frac{1}{0}\right) \geq 3, \Delta\left(\chi, \frac{1}{1}\right)=1$ for $\chi \in\{\alpha, \beta, \gamma, \delta\}$. Then $\Delta\left(\chi, \frac{0}{1}\right) \geq$ 2. By Lemma $7.1, \tilde{\mathcal{Q}}\left(\alpha, \beta, \gamma, \frac{0}{1}\right)$ is a lens space. Since $\Delta\left(\delta, \frac{0}{1}\right) \geq 2$, $[4$, Cyclic Surgery Theorem] implies that $K_{D}$ is an unknot or torus knot. By Lemma 8.3 , we have $\Delta\left(\gamma, \frac{1}{2}\right)=1$. Since $\Delta\left(\gamma, \frac{1}{1}\right)=1$ and $\Delta\left(\gamma, \frac{1}{0}\right) \geq 3$, $\gamma=2 / 3$.

By symmetry $\alpha=\beta=\gamma=\delta=2 / 3$. But $\mathcal{Q}\left(\frac{2}{3}, \frac{2}{3}, \frac{2}{3}, \frac{2}{3}\right)$ is a two component link.

Theorem 8.9. If $\mathcal{Q}(\alpha, \beta, \gamma, \delta)$ is the unknot then we cannot have $\Delta\left(\chi, \frac{1}{0}\right) \geq$ 3 for every $\chi \in\{\alpha, \beta, \gamma, \delta\}, \Delta\left(\alpha, \frac{0}{1}\right)=\Delta\left(\beta, \frac{0}{1}\right)=1$, and $\Delta\left(\gamma, \frac{1}{1}\right)=\Delta\left(\delta, \frac{1}{1}\right)=$ 1. 
Proof. Assume not. By Lemma 7.1, $\tilde{\mathcal{Q}}\left(\alpha, \beta, \gamma, \frac{0}{1}\right)$ is a lens space. On the other hand the distance assumptions imply that $\Delta\left(\delta, \frac{0}{1}\right) \geq 2$. Thus $[4$, Cyclic Surgery Theorem] forces $K_{D}$ to be an unknot or torus knot. By Lemma 8.3 we have $\Delta\left(\gamma, \frac{1}{2}\right)=1$. Together with the assumptions $\Delta\left(\gamma, \frac{1}{1}\right)=1, \Delta\left(\gamma, \frac{1}{0}\right) \geq$ 3 , we get that $\gamma=2 / 3$.

Applying the symmetry $(B, A, D, C)$, we conclude that $\delta=2 / 3$.

By Lemma 7.2 under the symmetry $(D, C, B, A), \tilde{\mathcal{Q}}\left(\frac{1}{1}, \beta, \gamma, \delta\right)$ is a lens space. Yet the given assumptions on distance mean that $\Delta\left(\alpha, \frac{1}{1}\right) \geq 2$. By $[4$, Cyclic Surgery Theorem], $K_{A}$ must be an unknot or torus knot. Lemma 8.3 under $(D, C, B, A)$ implies that $\Delta\left(\beta, \frac{1}{2}\right)=1$. Since $\Delta\left(\beta, \frac{0}{1}\right)=1, \Delta\left(\beta, \frac{1}{0}\right) \geq 3$, we see that $\beta=1 / 3$.

Applying the symmetry $(B, A, D, C), \alpha=1 / 3$. But $\mathcal{Q}\left(\frac{1}{3}, \frac{1}{3}, \frac{2}{3}, \frac{2}{3}\right)$ is a 2-component link.

\section{Appendix.}

In this appendix, we give the technical result proven by the arguments of Sections 3, 4, and 5-Theorem A.1. We then use this to classify the hyperbolic knots in solid tori which admit non-integral, toroidal surgeries Corollary A.2.

Theorem A.1. Let $M$ be an orientable, irreducible 3-manifold containing a separating, essential (i.e., incompressible and not boundary parallel) 2-torus $\widehat{T}$. Let $K$ be a knot in $M$ which intersects $\widehat{T}$ in two points and such that $E(K)$ contains no essential 2-sphere or 2-torus. Let $\widehat{Q}$ be a properly embedded, orientable surface in $K(\alpha)$ which intersects $K_{\alpha}$, a core of the attached solid torus in $K(\alpha)$, transversely. Let $Q=\widehat{Q} \cap E\left(K_{\alpha}\right), T=\widehat{T} \cap E(K)$, and let $G_{T}$, $G_{Q}$ be the graphs of intersection as described in Section 2. Assume that $G_{T}$ and $G_{Q}$ contain no 1-sided faces (i.e., trivial arcs of intersection). Assume also that $G_{Q}$ contains a great web $\Lambda$ such that all ghost labels are extremal with respect to $\Lambda$.

If $\Delta(\alpha, \tau)=2$, where $\tau$ is the meridian of $K$ in $M$, then $M$ is the union along $\widehat{T}$ of two Seifert fiber spaces, $M_{1}$ and $M_{2}$. Furthermore, each $M_{i}$ is a Seifert fiber space over either

a) a disk with two exceptional fibers;

or b) an annulus with one exceptional fiber.

In addition, a Seifert fiber from $M_{1}$ and one from $M_{2}$ intersect once on $\widehat{T}$.

Finally, $E(K)$ is obtained by Dehn filling the appropriate number of components of $E(L)$, where $L$ is as in Figure 5.24, where the component $K_{0}$ of 
$L$ corresponds to $K$, and $\tau=\frac{1}{0}, \alpha=\frac{1}{2}$ in the meridian-longitude framing of $K_{0}$.

Proof. Apply the arguments of Sections 3, 4, and 5 to $Q, T, G_{Q}, G_{T}$. Note that Lemma 3.3 still holds in this context. Use the fact that $E(K)$ is atoroidal and irreducible to conclude that any 2-torus in $E(K)$ either bounds a solid torus or is parallel to a boundary component.

Corollary A.2. Let $N$ be a solid torus and $K$ a knot in $N$ such that $K(\tau)$ contains an essential, separating 2-torus, $\widehat{T}$. Assume that $\Delta(\tau, \mu)>1$, where $\mu$ is the meridian of $K$, and that $E(K)$ is irreducible and atoroidal. Then $M=K(\tau)$ is a union of two Seifert fiber spaces $M_{1}, M_{2}$ along $\widehat{T}$, whose Seifert fibers intersect once on $\widehat{T}$. Furthermore, $M_{1}$ is Seifert fibered over the disk with two exceptional fibers and $M_{2}$ is Seifert fibered over the annulus with one exceptional fiber. One of the exceptional fibers of $M_{1}$ or $M_{2}$ has order 2. Finally, $(N, K) \cong\left(L\left(\alpha, \beta, \gamma, *, \frac{1}{2}\right), K_{0}\right)$ and $\left(M, K_{\tau}\right) \cong\left(L\left(\alpha, \beta, \gamma, *, \frac{1}{0}\right), K_{0}\right)$ for some $\alpha, \beta, \gamma$, where $K_{0}, L$ are as in Figure 5.24. Indeed, $K \subset N$ is obtained from an Eudave-Muñoz knot $k(\ell, m, n, p)$ by deleting the preimage of some tangle ball $(\tilde{R}(\chi)$ in the terminology of this paper) which is unknotted in $S^{3}$.

Proof. By [17], $\Delta(\tau, \mu)=2$ and there is an essential torus $\widehat{T}$ in $K(\tau)$ which intersects $K_{\tau}$, the core of the attached solid torus, exactly two times. Let $T=\widehat{T} \cap E\left(K_{\tau}\right)$ be the corresponding twice-punctured torus. Let $\widehat{Q}$ be a meridional disk of the solid torus $K(\mu)=N$ which intersects $K$ transversely and minimally. Let $Q=\widehat{Q} \cap E(K)$ be the corresponding punctured disk. In this context $\left(E(K)\right.$ is irreducible and atoroidal) we have that $G_{Q}, G_{T}$ contain no 1-sided faces. The proof of Theorem 2.1 goes through exactly as in Section 2 (indeed, the first line takes $G_{Q}$ to lie in a disk) to imply that $G_{Q}$ contains a great web $\Lambda$ whose ghost labels are extremal with respect to $\Lambda$.

Thus by Theorem A.1, $K(\tau)=M$ is the union of two Seifert fiber spaces as described. Further $E(K)$ is obtained by Dehn filling $E(L)$ along three of the boundary components corresponding to a 3-component sublink of $L$. By symmetry we may take $(N, K)=\left(L\left(\alpha, \beta, \gamma, *, \frac{1}{2}\right), K_{0}\right)$ and $\left(M, K_{\tau}\right)=$ $\left(L\left(\alpha, \beta, \gamma, *, \frac{1}{0}\right), K_{0}\right)$. Note that the latter implies that $\Delta\left(\chi, \frac{0}{1}\right) \geq 2$ for $\chi \in$ $\{\alpha, \beta, \gamma\}$.

Claim. There exists $\delta$ such that $L\left(\alpha, \beta, \gamma, \delta, \frac{1}{2}\right)=S^{3}$ and $\Delta\left(\delta, \frac{0}{1}\right)>2$.

Proof. Let $\varphi$ be the slope on $\partial L\left(\alpha, \beta, \gamma, *, \frac{1}{2}\right)$ of the boundary of the 
meridional disk. Note that $\frac{0}{1}$ is the slope of the Seifert fiber of $M_{2}$ on $\partial L\left(\alpha, \beta, \gamma, *, \frac{1}{0}\right)$, using the meridian-longitude framing of $L$. Assume that $\varphi=\frac{0}{1}$. Then $L\left(\alpha, \beta, \gamma, \frac{0}{1}, \frac{1}{2}\right)$ is $S^{2} \times S^{1}$ and $L\left(\alpha, \beta, \gamma, \frac{0}{1}, \frac{1}{0}\right)$ is either a lens space or the connected sum of two lens space (here a lens space does not include $S^{3}$ or $\left.S^{2} \times S^{1}\right)$. By Lemma 8.1, $L\left(\alpha, \beta, \gamma, \frac{0}{1}, *\right)$ is reducible. Since $L\left(\alpha, \beta, \gamma, \frac{0}{1}, \frac{1}{2}\right)$ is $S^{2} \times S^{1}$, which does not contain a separating essential 2sphere, $L\left(\alpha, \beta, \gamma, \frac{0}{1}, *\right)$ must contain $S^{2} \times S^{1}$ as a connected summand. But $L\left(\alpha, \beta, \gamma, \frac{0}{1}, \frac{1}{0}\right)$ contains no non-separating 2 -spheres. Thus $\varphi \neq \frac{0}{1}$.

Let $\delta$ be a slope satisfying $\Delta(\delta, \varphi)=1$ and $\Delta\left(\delta, \frac{0}{1}\right)>2$. Then $L\left(\alpha, \beta, \gamma, \delta, \frac{1}{2}\right)=S^{3}$, and $L\left(\alpha, \beta, \gamma, \delta, \frac{1}{0}\right)$ is the union of two Seifert fiber spaces, each over a disk with two exceptional fibers.

(Claim)

As in Section 6 , the quotient of $L(\alpha, \beta, \gamma, \delta, *)$ under the strong inversion of $L$ is $\mathcal{P}\left(\alpha^{\prime}, \beta^{\prime}, \gamma^{\prime}, \delta^{\prime}, *\right)$. Thus $\mathcal{P}\left(\alpha^{\prime}, \beta^{\prime}, \gamma^{\prime}, \delta^{\prime}, \frac{2}{1}\right)$ is the unknot and $\mathcal{P}\left(\alpha^{\prime}, \beta^{\prime}, \gamma^{\prime}, \delta^{\prime}, \frac{0}{1}\right)$ is the union of prime tangles $\left(\Delta\left(\chi, \frac{1}{0}\right) \geq 2\right.$ for $\chi \in$ $\left.\left\{\alpha^{\prime}, \beta^{\prime}, \gamma^{\prime}, \delta^{\prime}\right\}\right)$. Now Proposition 6.2 says that $\mathcal{P}\left(\alpha^{\prime}, \beta^{\prime}, \gamma^{\prime}, \delta^{\prime}, *\right)$ is one of the tangles $B(\ell, m, n, p)$ of [6]. That is, $K_{0} \subset L\left(\alpha, \beta, \gamma, \delta, \frac{1}{2}\right)$ is an Eudave-Muñoz knot.

Thus $(N, K)=\left(L\left(\alpha, \beta, \gamma, *, \frac{1}{2}\right), K_{0}\right)$ comes from an Eudave-Muñoz knot as claimed. Finally, by Proposition 6.2, $\frac{1}{2} \in\left\{\alpha^{\prime}, \beta^{\prime}, \gamma^{\prime}, \delta^{\prime}\right\}$. That is, $\Delta\left(\chi, \frac{0}{1}\right)=2$ for some $\chi \in\{\alpha, \beta, \gamma\}$, since the longitude framing of $L$ corresponds to the tangle framing $\frac{1}{0}$ of $\mathcal{P}$ and $\Delta\left(\delta, \frac{0}{1}\right)>2$. This means that one of the exceptional fibers of $M_{1}$ or $M_{2}$ must have order 2 .

\section{References.}

[1] J. Berge, Obtaining lens spaces by surgery on knots, unpublished manuscript.

[2] S. Bleiler and C. Hodgson, Spherical space forms and Dehn surgery, Topology 35 (1996), 809-833.

[3] S. Boyer and X. Zhang, Culler-Shalen semi-norms and Dehn fillings, Ann. of Math. 148 (1998), 1-66.

[4] M. Culler, C. McA. Gordon, J. Luecke, and P. Shalen, Dehn surgery on knots, Ann. of Math. (2) 125 (1987), no. 2, 237-300.

[5] J. Dean, Small Seifert-fibered Dehn surgery on hyperbolic knots, Alg. Geom. Top. 3 (2003), 435-472.

[6] M. Eudave-Muñoz, Non-hyperbolic manifolds obtained by Dehn surgery on hyperbolic knots, Proceedings of the 1993 International Georgia 
Topology Conference, W. Kazez (Ed.), AMS/IP Stud. in Adv. Math., Vol.2, AMS, Providence, RI, 1997, pp. 35-61.

[7] M. Eudave-Muñoz, On hyperbolic knots with Seifert fibered surgeries, Topology Appl. 121 (2002), 119-141.

[8] D. Gabai, Foliations and the topology of 3-manifolds. III, J. Differential Geometry 26 (1987), 479-536.

[9] F. Gonzalez-Acuña and H. Short, Knots surgery and primeness, Math. Proc. Camb. Phil. Soc. 99 (1986), 89-102.

[10] C. McA. Gordon, Dehn surgery and satellite knots, Trans. Amer. Math. Soc. 275 (1983), 687-708.

[11] C. McA. Gordon and R.A. Litherland, Incompressible planar surfaces in 3-manifolds, Topology Appl. 18 (1984), 121-144.

[12] C. McA. Gordon and J. Luecke, Knots are determined by their complements, J. Amer. Math. Soc. 2 (1989), 371-415.

[13] C. McA. Gordon and J. Luecke, Only integral Dehn surgeries can yield reducible manifolds, Math. Proc. Camb. Phil. Soc. 102 (1987), 94-101.

[14] C. McA. Gordon and J. Luecke, Reducible manifolds and Dehn surgery, Topology 35 (1996), 385-409.

[15] C. McA. Gordon and J. Luecke, Dehn surgeries on knots creating essential tori, I, Comm. Anal. Geom. 3 (1995), 597-644.

[16] C. McA. Gordon and J. Luecke, Dehn surgeries on knots creating essential tori, II, Comm. Anal. Geom. 8 (2000), no. 4, 671-725.

[17] C. McA. Gordon and J. Luecke, Toroidal and boundary-reducing Dehn fillings, Topology Appl. 93 (1999), 77-90.

[18] W. Haken, Some results on surfaces in 3-manifolds, Studies in Modern Topology (Math. Assoc. Amer., distributed by Prentice-Hall, 1968) 3498.

[19] T. Mattman, K. Miyazaki and K. Motegi, Seifert fibered surgeries which do not arise from primitive/Seifert-fibered constructions, preprint.

[20] K. Miyazaki and K. Motegi, Toroidal and annular Dehn surgeries of solid tori, Topology Appl. 93 (1999), 173-178. 
[21] M. Scharlemann, Producing reducible manifolds by surgery on a knot, Topology 29 (1990), 481-500.

[22] M. Teragaito, Creating Klein bottles by surgery on knots, J. Knot Theory Ramifications 10 (2001), 781-794. 